\title{
Nilpotent networks and 4D RG flows
}

\author{
Fabio Apruzzi ${ }^{a, b}$ Falk Hassler, ${ }^{a, b}$ Jonathan J. Heckman ${ }^{a}$ and Thomas B. Rochais ${ }^{a}$ \\ ${ }^{a}$ Department of Physics and Astronomy, University of Pennsylvania, \\ Philadelphia, PA 19104, U.S.A. \\ ${ }^{b}$ Department of Physics, University of North Carolina, \\ Chapel Hill, NC 27599, U.S.A. \\ E-mail: fabio.apruzzi@gmail.com, fhassler@unc.edu, \\ jheckman@sas.upenn.edu, thb@sas.upenn.edu
}

ABSTRACT: Starting from a general $\mathcal{N}=2$ SCFT, we study the network of $\mathcal{N}=1$ SCFTs obtained from relevant deformations by nilpotent mass parameters. We also study the case of flipper field deformations where the mass parameters are promoted to a chiral superfield, with nilpotent vev. Nilpotent elements of semi-simple algebras admit a partial ordering connected by a corresponding directed graph. We find strong evidence that the resulting fixed points are connected by a similar network of $4 \mathrm{D}$ RG flows. To illustrate these general concepts, we also present a full list of nilpotent deformations in the case of explicit $\mathcal{N}=2 \mathrm{SCFTs}$, including the case of a single D3-brane probing a $D$ - or $E$-type F-theory 7-brane, and 6D $(G, G)$ conformal matter compactified on a $T^{2}$, as described by a single M5-brane probing a $D$ - or $E$-type singularity. We also observe a number of numerical coincidences of independent interest, including a collection of theories with rational values for their conformal anomalies, as well as a surprisingly nearly constant value for the ratio $a_{\mathrm{IR}} / c_{\mathrm{IR}}$ for the entire network of flows associated with a given UV $\mathcal{N}=2 \mathrm{SCFT}$. The arXiv submission also includes the full dataset of theories which can be accessed with a companion Mathematica script.

Keywords: Anomalies in Field and String Theories, Conformal Field Theory, Renormalization Group

ARXIV EPRINT: 1808.10439 


\section{Contents}

1 Introduction 1

2 Nilpotent deformations: generalities 5

$\begin{array}{ll}2.1 & \text { Illustrative Lagrangian example }\end{array}$

3 Inherited infrared symmetries $\quad 12$

$\begin{array}{ll}3.1 & \text { Plain mass deformations } \\ & 13\end{array}$

$\begin{array}{ll}3.1 .1 & \text { Operator scaling dimensions } \\ & 16\end{array}$

$\begin{array}{ll}3.1 .2 & \text { Monotonicity } \\ & 16\end{array}$

$\begin{array}{lll}3.2 & \text { Flipper field deformations } & 17\end{array}$

4 Emergent symmetries and operator decoupling $\quad 19$

4.1 Decoupling of Coulomb branch operators 20

4.2 Decoupling of mesonic operators 21

4.3 Rational theories 24

4.4 Ordering of RG flows 24

5 D3-brane probe theories $\quad 25$

5.1 Summary of UV $\mathcal{N}=2$ fixed points 26

$\begin{array}{ll}5.2 & \text { Plain mass deformations } \\ 5.3 & 26\end{array}$

$\begin{array}{lll}5.3 & \text { Flipper field deformations } & 29\end{array}$

$6 \quad 4 \mathrm{D}$ conformal matter theories $\quad 31$

6.1 Summary of UV $\mathcal{N}=2$ fixed points 32

6.2 Plain mass deformations 33

6.3 Flipper field deformations 36

$\begin{array}{lll}7 & \text { Conclusions } & 36\end{array}$

A The embedding index $\quad 39$

B From 6D to 4D conformal matter 42

C Accessing the complete tables 46

\section{Introduction}

Conformal field theories (CFTs) play a central role in physics. Deformations which drive one fixed point to another also provide important insights into more general quantum field theories.

Even so, it is often difficult to establish the existence of fixed points, let alone determine deformations to new ones. Common techniques include combinations of methods from supersymmetry, string compactification, holography, and / or the conformal bootstrap. 
Part of the issue with understanding relevant perturbations of CFTs is that (by definition) they grow deep in the infrared. From this perspective, it is perhaps not surprising that comparatively short flows where there is only a small drop in the number of degrees of freedom (as measured by various anomalies) are often easier to study.

One way to understand long flows is to break them up into a sequence of nearby short flows. This strategy has recently been used to make surprisingly sharp statements in the study of $6 \mathrm{D}$ supersymmetric $\mathrm{RG}$ flows [1-7]. In particular, the mathematical partial ordering of nilpotent orbits in flavor symmetry algebras automatically defines a hierarchy of $6 \mathrm{D}$ RG flows [5-7]. For a recent review of $6 \mathrm{D}$ superconformal field theories, see reference [8].

In this paper we ask whether the same mathematical structure leads to an improved understanding of RG flows in lower-dimensional systems. The specific class of theories we study are $\mathcal{N}=1$ deformations of $4 \mathrm{D} \mathcal{N}=2$ SCFTs. For the UV theories under consideration, we assume the existence of a flavor symmetry algebra $\mathfrak{g}_{\text {flav }}$, which a priori could be composed of several simple factors:

$$
\mathfrak{g}_{\text {flav }}=\mathfrak{g}_{\text {flav }}^{(1)} \times \ldots \times \mathfrak{g}_{\text {flav }}^{(n)}
$$

for $\mathfrak{g}_{\text {flav }}^{(i)}$ a simple Lie algebra. Associated with this flavor symmetry are a collection of mass parameters $m_{\text {adj }}$, and corresponding dimension two mesonic operators $\mathcal{O}_{\text {adj }}$ transforming in the adjoint representation, ${ }^{1}$ which can be used to activate relevant deformations to new conformal fixed points in the IR via superpotential deformations:

$$
\delta W=\operatorname{Tr}_{\mathfrak{g}_{\text {flav }}}\left(m_{\mathrm{adj}} \cdot \mathcal{O}_{\mathrm{adj}}\right) .
$$

Promoting the mass parameters to a chiral superfield $M_{\text {adj }}$ transforming in the adjoint representation of $\mathfrak{g}_{\text {flav }}$, we can consider the related deformations associated with expanding around background vevs for these "flipper fields:"

$$
\delta W=\operatorname{Tr}_{\mathfrak{g}_{\text {flav }}}\left(\left(m_{\text {adj }}+M_{\text {adj }}\right) \cdot \mathcal{O}_{\text {adj }}\right),
$$

where now, we interpret the mass deformation $m_{\text {adj }}=\left\langle M_{\text {adj }}\right\rangle$ as a background vev.

The key point we shall be exploiting in this work is that given a flavor symmetry Lie algebra $\mathfrak{g}_{\text {flav }}$, there is a partial ordering available for nilpotent elements, as defined by the orbit of an element under the adjoint action of the algebra. Given nilpotent elements $\mu, \nu \in \mathfrak{g}_{\text {flav }}$, we say that $\mu \prec \nu$ when $\operatorname{Orbit}(\mu) \subset \overline{\operatorname{Orbit}(\nu)}$. Since the mass parameters $m_{\text {adj }}$ transform in the adjoint, this sets up a conjectural relation between relevant deformations, as in lines (1.2) and (1.3) and 4D RG flows. Intuitively, as the size of the orbit increases, the number of degrees of freedom which pick up a mass also increases, leading to a longer flow into the infrared.

Another quite interesting feature of nilpotent mass deformations is that at least in the case where we have a plain mass deformation as in line (1.2), the Seiberg-Witten curve of the UV $\mathcal{N}=2$ theory descends to an $\mathcal{N}=1$ curve of the deformed $\mathcal{N}=1$ theory which fixes the relative scaling dimensions of various operators [9]. The fact that it is still singular provides evidence of an $\mathcal{N}=1$ fixed point.

\footnotetext{
${ }^{1}$ More canonically, one can view the mass parameters as elements in the dual $\mathfrak{g}_{\text {flav }}^{*}$.
} 
One of our aims in this work will be to provide substantial evidence that this network of nilpotent orbits defines a corresponding hierarchy of 4D RG flows. For the most part, this involves a mild generalization of the procedure proposed in [10], studied in detail in [9] (see also [11]) and further extended in references [12-18], and applied in various model building contexts in references [19-24].

The appearance of a nilpotent element $\mu$ implies the existence of an $\mathfrak{s u}(2) \subset \mathfrak{g}_{\text {flav }}$ subalgebra, with generators $\mu, \mu^{\dagger}$ and $\left[\mu, \mu^{\dagger}\right]$. Labelling the associated generator of the Cartan subalgebra for this $\mathfrak{s u}(2)$ subalgebra as $T_{3}$, the infrared R-symmetry is given by a linear combination of the form (see e.g. [9]):

$$
R_{\mathrm{IR}}=R_{\mathrm{UV}}+\left(\frac{t}{2}-\frac{1}{3}\right) J_{\mathcal{N}=2}-t T_{3}+\sum_{i} t_{i} F_{i},
$$

where $R_{\mathrm{UV}}$ and $R_{\mathrm{IR}}$ respectively denote the UV and IR R-symmetry (treated as an $\mathcal{N}=1$ theory), $J_{\mathcal{N}=2}$ is an additional $\mathrm{U}(1)$ symmetry which is always present in an $\mathcal{N}=2$ SCFT when interpreted as an $\mathcal{N}=1$ theory. The last set of terms refers to the possibility of additional U(1)'s, including those which emerge in the infrared. The IR R-symmetry is then fixed via the procedure of a-maximization over the parameters $t$ and $t_{i}$, as in reference [25]. ${ }^{2}$

In the absence of these emergent U(1)'s, we find strong evidence that the partially ordered set defined by the nilpotent elements of a Lie algebra exactly aligns with the corresponding hierarchy of 4D RG flows. For example, the conformal anomalies $a_{\mathrm{IR}}$ and $c_{\mathrm{IR}}$ decrease along such trajectories, and anomalies involving flavor currents (with generators suitably normalized) also decrease along such flows.

Far more non-trivial is that even in the presence of emergent $U(1)$ 's, there is still such a partial ordering of $4 \mathrm{D}$ theories, as dictated by the nilpotent cone of the Lie algebra. This is considerably more subtle and requires a case by case analysis. For this reason, we focus on explicit examples.

One class of theories already studied in [9] for plain mass deformations, and with some masses promoted to chiral superfields in $[16,17]$ involves nilpotent mass deformations of the $\mathcal{N}=2$ theories defined by a D3-brane probing an F-theory 7-brane with constant axio-dilaton. This includes the $H_{0}, H_{1}, H_{2}$ Argyres-Douglas theories [26, 27], the $E_{6}, E_{7}$, $E_{8}$ Minahan Nemeschansky theories [28, 29], and $\mathcal{N}=2 \mathrm{SU}(2)$ gauge theory with four flavors and corresponding $\mathrm{SO}(8)$ flavor symmetry (namely $D_{4}$ ) [30]. The string theory interpretation of nilpotent deformations is also quite interesting, as they are associated with T-brane configurations of 7-branes (see e.g. [11, 31-41]), namely they leave intact the Weierstrass model of the associated F-theory geometry, but nevertheless deform the physical theory.

Here, we systematically study all possible nilpotent deformations for the $D$ - and $E$ series theories, systematically sweeping out the corresponding network of $4 \mathrm{D}$ RG flows (we do not consider the $H$-series in any detail since they have only a few nilpotent deformations). An interesting feature of these examples is that only the Coulomb branch operator

\footnotetext{
${ }^{2}$ In practice it is often necessary to make additional assumptions about these emergent symmetries to actually carry out concrete calculations.
} 
sometimes appears to drop below the unitarity bound, and even this happens only for the largest nilpotent orbits. In such cases, we see no evidence that the fixed point does not exist (since the underlying geometry is still singular), and instead find it most plausible that the Coulomb branch operator decouples as a free field, with a corresponding emergent $\mathrm{U}(1)$ acting on only this operator, as per the procedure advocated in [42, 43].

We also study nilpotent mass deformations of $4 \mathrm{D} \mathcal{N}=2$ conformal matter, namely the compactification of $6 \mathrm{D}$ conformal matter $[44,45]$ on a $T^{2}$. Here, we consider the case where there is a $G_{L} \times G_{R}$ flavor symmetry with $G_{L}=G_{R}=G$ given by $\mathrm{SO}(8), E_{6}, E_{7}$, or $E_{8}$. The $4 \mathrm{D}$ anomaly polynomials for these theories were computed in [46, 47]. The Seiberg-Witten and Gaiotto curves for these models are known, both via mirror symmetry [48], and via its relation to compactifications of class $\mathcal{S}$ theories [46, 47].

Nilpotent mass deformations of $4 \mathrm{D}$ conformal matter involve specifying a pair of nilpotent elements, one for each flavor symmetry factor. In this case, the string theory interpretation involves a pair of 7-branes intersecting along the common $T^{2}$. Such nilpotent deformations involve activating background values for gauge fields of the corresponding 7-branes.

This already leads to many new $\mathcal{N}=1$ fixed points and the partial ordering for the product Lie algebra predicts a corresponding hierarchy of $4 \mathrm{D}$ fixed points. We present strong evidence that this is the case, again sweeping over all pairs of nilpotent orbits, and for each one computing the corresponding values of various IR anomalies, checking there is a corresponding decrease along a given trajectory in the nilpotent cone.

One issue which shows up in these cases is that in sufficiently long flows, mesonic operators often decouple. This in turn signals that such operators cannot be used to trigger further flows. A priori, this could mean that the network of connections in the nilpotent cone may have links which do not produce 4D RG flows. Even though we have not found a single example where this actually occurs, we leave a systematic analysis of this possibility for future work.

With this set of theories in hand, additional numerical studies are amenable to treatment, though the list of theories is so large that we have chosen to collect the full dataset in an accompanying Mathematica package available for download with our arXiv submission. For example, by sweeping over all theories, we find several examples of theories where the conformal anomalies $a_{\mathrm{IR}}$ and $c_{\mathrm{IR}}$ are rational numbers. In some cases such as reference $[16,17]$, this was interpreted as evidence for an emergent $\mathcal{N}=2$ supersymmetry in the infrared, and we find another example of this type for a deformation of the $E_{7}$ MinahanNemeschansky theory. It is not clear to us whether there is $\mathcal{N}=2$ enhancement in all cases, but certainly the list of such rational theories we find suggests additional structure is present. Another numerical curiosity we observe is that for a given choice of UV $\mathcal{N}=2$ SCFT, the value of the ratio:

$$
\frac{a_{\mathrm{IR}}}{c_{\mathrm{IR}}} \simeq \text { constant } \pm O(1 \%-5 \%)
$$

is nearly constant over all nilpotent deformations, in line with the observation made in reference [49] for a different set of theories.

The rest of this paper is organized as follows. First, in section 2 we analyze for a general $\mathcal{N}=2$ theory with flavor symmetries, the structure of the $\mathcal{N}=1$ theories obtained via both 


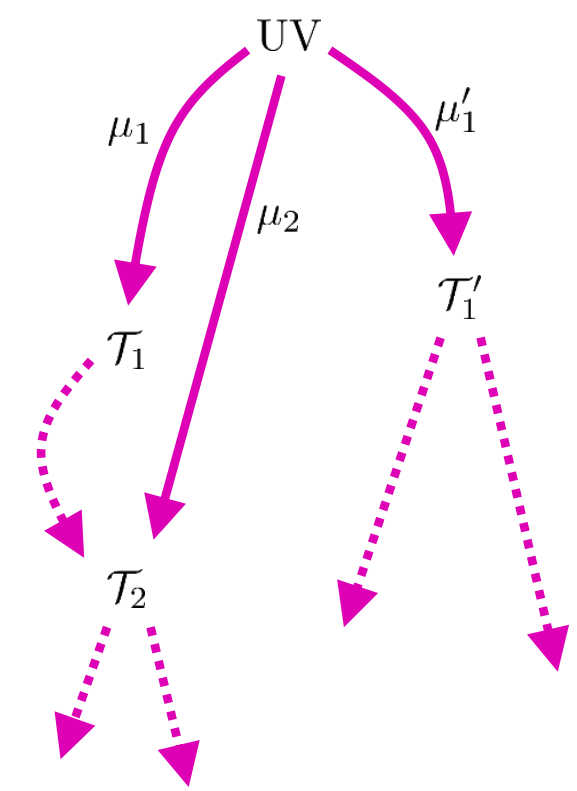

Figure 1. Depiction of the network of $4 \mathrm{D}$ RG flows generated by elements of the nilpotent cone. Starting from a UV $\mathcal{N}=2$ fixed point, each nilpotent orbit in the flavor symmetry algebra determines a candidate $\mathcal{N}=1$ fixed point. Additionally, the network of connections between nilpotent orbits also motivates the existence of additional flows between these $\mathcal{N}=1$ fixed points.

plain mass deformations and their extension to flipper field deformations. In particular, we analyze the network of 4D RG flows predicted by the nilpotent cone. Section 3 discusses the structure of IR fixed points assuming no operators decouple, and section 4 discusses the structure of theories in the presence of emergent IR symmetries. In section 5 we discuss nilpotent deformations of D3-brane probes of $D$ - and $E$-type 7-branes and in section 6 we discuss nilpotent deformations of $4 \mathrm{D} \mathcal{N}=2$ conformal matter. We conclude in section 7 . Some additional review material, as well as technical details and instructions on how to use the companion Mathematica files are presented in the appendices.

\section{Nilpotent deformations: generalities}

In this section we discuss some general features of nilpotent mass deformations of $\mathcal{N}=2$ SCFTs. Throughout, we assume the existence of a continuous flavor symmetry algebra which may consist of several simple factors:

$$
\mathfrak{g}_{\mathrm{UV}} \equiv \mathfrak{g}_{\text {flav }}=\mathfrak{g}_{\text {flav }}^{(1)} \times \ldots \times \mathfrak{g}_{\text {flav }}^{(n)} .
$$

We assume either that there are no abelian factors in the UV, or more generally, that the only non-vanishing anomalies involving flavor symmetry currents involve precisely two insertions of the same kind (which is automatic in the traceless non-abelian case). Note that we can then also allow the appearance of abelian symmetry factors, provided they satisfy this condition.

We assume adjoint valued mass parameters $m_{\mathrm{adj}}$, and corresponding dimension two mesonic operators $\mathcal{O}_{\text {adj }}$ which serve as coordinates on the Higgs branch of moduli space. 


\begin{tabular}{|c|c|c|}
\hline & $\mathcal{O}_{\text {adj }}$ & $Z_{i}$ \\
\hline$R_{\mathrm{UV}}$ & $4 / 3$ & $2 / 3 \Delta_{\mathrm{UV}}\left(Z_{i}\right)$ \\
\hline$J_{\mathcal{N}=2}$ & -2 & $2 \Delta_{\mathrm{UV}}\left(Z_{i}\right)$ \\
\hline$R_{\mathcal{N}=2}$ & 0 & $2 \Delta_{\mathrm{UV}}\left(Z_{i}\right)$ \\
\hline$I_{3}$ & 1 & 0 \\
\hline
\end{tabular}

Table 1. Charge assignments for the mesons $\mathcal{O}_{\text {adj }}$ and Coulomb branch parameters $Z_{i}$ in the UV theory.

Note that there could be non-trivial chiral ring relations for these operators, as can often happen when there is more than one simple Lie algebra factor for $\mathfrak{g}_{\mathrm{UV}}$. Since we will couch our analysis in terms of basic properties of symmetry breaking patterns, our analysis will not depend on such detailed knowledge of the UV theory.

It will prove useful to view our $\mathcal{N}=2 \mathrm{SCFT}$ as an $\mathcal{N}=1 \mathrm{SCFT}$ with additional symmetries. Along these lines, we recall that the $\mathcal{N}=2$ SCFT has an $\mathrm{SU}(2) \times \mathrm{U}(1) \mathrm{R}$ symmetry. Labelling the generator of the Cartan subalgebra for the $\mathrm{SU}(2)$ factor by $I_{3}$ with eigenvalues $\pm 1 / 2$ in the fundamental representation, and $R_{\mathcal{N}=2}$ for the $\mathrm{U}(1)$ factor normalized so that the complex scalar of a free $\mathcal{N}=2$ vector multiplet has charge +2 , the $\mathcal{N}=1 \mathrm{R}$-symmetry is given by the linear combination (see e.g $[9,50]$ ):

$$
R_{\mathrm{UV}}=\frac{1}{3} R_{\mathcal{N}=2}+\frac{4}{3} I_{3}
$$

There is another linear combination which we can form which is a global symmetry of the UV theory. We label this as:

$$
J_{\mathcal{N}=2}=R_{\mathcal{N}=2}-2 I_{3}
$$

See table 1 for the charge assignments of Coulomb branch operators and mesonic operators which serve as coordinates on the Higgs branch.

The Higgs branch is parameterized by dimension two operators transforming in the adjoint representation of $\mathfrak{g}_{\text {flav }} \equiv \mathfrak{g}_{\mathrm{UV}}$, which we denote by $\mathcal{O}_{\text {adj }}$. The mass parameters $m_{\text {adj }}$ which pair with these operators transform in the adjoint representation of $\mathfrak{g}_{\text {flav }}$.

We consider both the case of a plain mass deformation:

$$
\delta W_{\text {plain }}=\operatorname{Tr}_{\mathfrak{g}_{\text {flav }}}\left(m_{\text {adj }} \cdot \mathcal{O}_{\text {adj }}\right),
$$

as well as the flipper field deformations associated with promoting the mass parameters to a dynamical chiral superfield in the adjoint of the flavor symmetry which mixes with the original interacting theory:

$$
\delta W_{\text {flip }}=\operatorname{Tr}_{\mathfrak{g}_{\text {flav }}}\left(\left(m_{\mathrm{adj}}+M_{\mathrm{adj}}\right) \cdot \mathcal{O}_{\mathrm{adj}}\right) .
$$

We shall often first deal with the case of plain mass deformations, since flipper field deformations are a mild extension of this case (though the resulting IR physics can be quite different, see e.g. $[12,15-17])$. An important feature of our analysis is that the general 
structure of symmetries and anomalies enables us to give a uniform analysis of RG flows for many such relevant deformations.

Though it may be difficult to explicitly construct, we know that the IR physics on the Coulomb branch is controlled by a Seiberg-Witten curve [30, 51], and mass deformations enter as flavor symmetry neutral combinations constructed from the holomorphic Casimir invariants of $\mathfrak{g}_{\text {flav }}$. In the special case of an $\mathcal{N}=2 \mathrm{SCFT}$, all mass deformations have been switched off and this curve will exhibit singularities, as required to have massless degrees of freedom at the origin of the Coulomb branch.

We will in particular be interested in nilpotent deformations. For the classical algebras, these can always be presented in terms of an explicit nilpotent matrix, which upon conjugation by a complexified symmetry generator can always be taken to be proportional to a matrix in Jordan normal form. For example, in $\mathfrak{s u}(4)$ we have:

$$
\left[\begin{array}{cccc}
0 & m_{1 \overline{2}} & 0 & 0 \\
0 & 0 & m_{2 \overline{3}} & 0 \\
0 & 0 & 0 & m_{3 \overline{4}} \\
0 & 0 & 0 & 0
\end{array}\right] \sim m \times\left[\begin{array}{llll}
0 & 1 & 0 & 0 \\
0 & 0 & 1 & 0 \\
0 & 0 & 0 & 1 \\
0 & 0 & 0 & 0
\end{array}\right] .
$$

The labelling scheme for the classical $\mathfrak{s u}, \mathfrak{s p}$ and $\mathfrak{s o}$ algebras are dictated by its presentation as a direct sum of nilpotent Jordan blocks. These blocks in turn define a partition of an integer which we write as $\left[\mu_{1}^{a_{1}}, \ldots, \mu_{k}^{a_{k}}\right]$ with $\mu_{1}>\ldots>\mu_{k}>0$ and $a_{i}$ the multiplicity. In the case of $\mathfrak{s u}(N)$, each partition of the integer $N$ defines a nilpotent orbit. In the case of $\mathfrak{s o}(2 N)$, there are some additional restrictions on partitions of $2 N$, namely we require every even number in a partition to appear an even number of times. Similar considerations hold for $\mathfrak{s p}(N)$ and $\mathfrak{s o}(2 N+1)$. In the case of the exceptional algebras, we instead label the nilpotent orbit by its embedding in some subalgebra of the larger parent algebra, which is known as the Bala-Carter label.

Now, one of the very interesting features of nilpotent mass deformations is that all holomorphic Casimir invariants (by definition) must vanish, and so the presentation of the singular geometry is exactly the same as the $\mathcal{N}=2$ theory. In contrast to the $\mathcal{N}=2$ case, however, this does not mean it is possible to read absolute scaling dimensions of operators from the curve (see reference [27] for the analysis of $\mathcal{N}=2$ theories), but instead only the relative scaling dimensions of operators [9]. Nevertheless, the appearance of a singular curve provides one indication that we are still dealing with a conformal field theory, albeit one with reduced supersymmetry.

Assuming the existence of such a fixed point, there is a partial ordering of nilpotent orbits which suggests a physical ordering of theories. Given a pair of nilpotent elements $\mu$ and $\nu$, we say that $\mu \prec \nu$ when $\operatorname{Orbit}(\mu) \subset \overline{\operatorname{Orbit}(\nu)}$, where the overline denotes the Zariski closure of the orbit in $\mathfrak{g}_{\text {flav }}$.

Physically, the bigger the orbit, the more degrees of freedom have picked up a mass. So, it is natural to expect bigger orbits to be deeper in the infrared. Moreover, for each of the simple Lie algebras, there is a classification of all possible nilpotent orbits, and the associated containment relations for these choices. This partially ordered set and its interconnections defines a directed graph, namely the Hasse diagram of the nilpotent cone. 


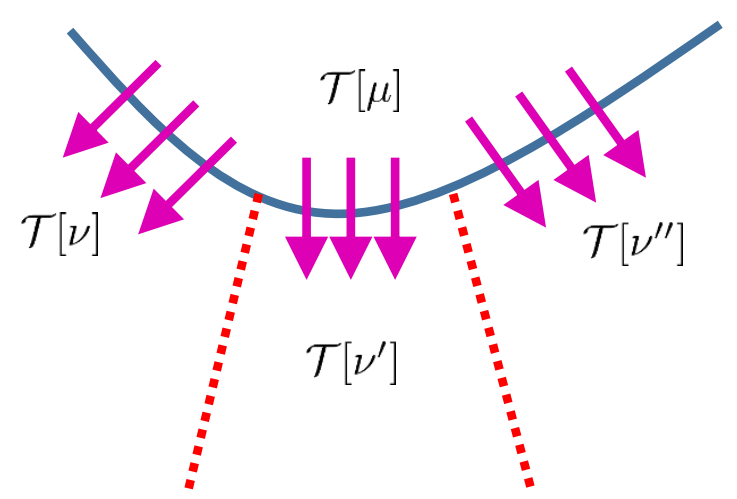

Figure 2. Depiction of the deformations from one nilpotent orbit to another. Here, we label a theory by a choice of nilpotent orbit $\mathcal{T}[\mu]$, and subsequent deformations deeper down in the nilpotent cone to theories $\mathcal{T}[\nu], \mathcal{T}\left[\nu^{\prime}\right]$ and $\mathcal{T}\left[\nu^{\prime \prime}\right]$. These physical paths to new orbits are parameterized by the remnants of the original mesonic operators. An important subtlety with this picture is that as we proceed from the UV to the IR, various mesonic operators may decouple, severing some of the candidate links between theories. In explicit examples, however, we have not observed this pathological behavior.

Returning to our example of explicit nilpotent matrices in $\mathfrak{s u}(4)$, for example, we can see a clear hierarchy:

$$
\left[\begin{array}{llll}
0 & 0 & 0 & 0 \\
0 & 0 & 0 & 0 \\
0 & 0 & 0 & 0 \\
0 & 0 & 0 & 0
\end{array}\right] \prec\left[\begin{array}{cccc}
0 & m_{1 \overline{2}} & 0 & 0 \\
0 & 0 & 0 & 0 \\
0 & 0 & 0 & 0 \\
0 & 0 & 0 & 0
\end{array}\right] \prec\left[\begin{array}{cccc}
0 & m_{1 \overline{2}} & 0 & 0 \\
0 & 0 & m_{2 \overline{3}} & 0 \\
0 & 0 & 0 & m_{3 \overline{4}} \\
0 & 0 & 0 & 0
\end{array}\right]
$$

It is tempting to also interpret this diagram as a collection of candidate $R G$ flows between $\mathcal{N}=1$ fixed points. Given a sequence of theories $\mathcal{T}_{\mathrm{UV}} \rightarrow \ldots \rightarrow \mathcal{T}_{i} \rightarrow \mathcal{T}_{i+1} \rightarrow \ldots$, and associated nilpotent orbits $\varnothing \prec \ldots \prec \mu_{i} \prec \mu_{i+1} \prec \ldots$, we can ask whether there is a flow directly from the intermediate $\mathcal{N}=1$ fixed point $\mathcal{T}_{i}$ to $\mathcal{T}_{i+1}$. Indeed, we can subtract the two deformations of the original parent theory:

$$
\delta W_{i \rightarrow i+1}=\operatorname{Tr}_{\mathfrak{g}_{\text {flav }}}\left(\left(\mu_{i+1}-\mu_{i}\right) \cdot \mathcal{O}_{\mathrm{adj}}\right)
$$

which is itself a relevant deformation of the UV fixed point theory. Assuming that the operators necessary to perform such a deformation do not decouple in theory $\mathcal{T}_{i}$, this strongly indicates that each link in the directed graph defined by the Hasse diagram also defines a flow between $\mathcal{N}=1$ fixed points. Carrying out a systematic analysis of this is somewhat subtle, especially when operators start to decouple in long flows, but this at least shows that the structure of the nilpotent cone leads to a rich network of $4 \mathrm{D}$ RG flows. See figure 2 for a depiction of the flows generated by these mesonic operators.

Let us now make more precise the sense in which operator deformations such as those of line (2.8) lead to perturbations of one fixed point to another. Along these lines, we start in some theory $\mathcal{T}[\mu]$, as characterized by $\operatorname{Orbit}(\mu)$. Given a nilpotent element, the JacobsonMorozov theorem guarantees the existence of a homomorphism $\mathfrak{s u}(2) \rightarrow \mathfrak{g}_{U V}$, and we label 
the generators of this algebra by $T_{3}, T_{+}$and $T_{-}$in the obvious notation. Decomposing the adjoint representation into irreducible representations of this $\mathfrak{s u}(2)$ subalgebra, we get:

$$
V_{\text {adj }}=\bigoplus_{j}(j),
$$

where we allow each spin $j$ to come with some multiplicity. The highest spin states of each representation specify the deformations of the nilpotent orbit. Indeed, a convenient way to compute the dimension of the orbit is via the formula:

$$
\operatorname{dim} \operatorname{Orbit}(\mu)=\operatorname{dim} V_{\mathrm{adj}}-\operatorname{dim} V_{0}-\operatorname{dim} V_{1 / 2},
$$

where here, we have decomposed the states of the adjoint representation under the $T_{3}$ grading:

$$
V_{\text {adj }}=\bigoplus_{s} V_{s}
$$

In the physical theory, these top spin states are distinguished by their role in the breaking pattern of the flavor symmetry. More formally, we begin with the $\mathcal{N}=1$ current supermultiplet for the flavor symmetry of the original theory $\mathcal{J}_{A}$, with $A$ an index in the adjoint representation. In the unbroken phase, we have the conservation rule:

$$
\bar{D}^{2} \mathcal{J}_{A}=0
$$

We can also track what becomes of this relation in the broken phase (after the mass deformation has been switched on). Since $\mathcal{J}_{A}$ transforms in the adjoint representation of $\mathfrak{g}_{\mathrm{UV}}$, we can decompose it into representations of this $\mathfrak{s u}(2)$ subalgebra, so we label it by a choice of spin $j$, and $T_{3}$ charge $s$, namely $\mathcal{J}_{j, s}$. In the broken phase, the current is not conserved, since it is explicitly broken by our mass deformation. We can follow the standard Noether procedure to see the source of the current non-conservation. Introducing a "pion" chiral superfield $\Lambda$ which parameterizes the flavor symmetry generators, we can send:

$$
\mathcal{O}_{\text {adj }} \rightarrow e^{i \Lambda} \mathcal{O}_{\text {adj }} e^{-i \Lambda}
$$

Then, the superpotential deformation transforms as:

$$
\delta W \rightarrow \operatorname{Tr}_{\mathfrak{g}_{\mathrm{UV}}}\left(m_{\mathrm{adj}} \cdot e^{i \Lambda} \mathcal{O}_{\mathrm{adj}} e^{-i \Lambda}\right),
$$

so since $m_{\text {adj }}$ can, without loss of generality, be taken to be the raising operator of the $\mathfrak{s u}(2)_{D}$ subalgebra, we learn that we instead have (see e.g. [16, 52]):

$$
-\frac{1}{4} \bar{D}^{2} \mathcal{J}_{j, s}=\mathcal{O}_{j, s-1}
$$

Note in particular the relative shift in the $T_{3}$ charge $s$.

As explained in $[16,52]$, this relation tells us that in the perturbed chiral ring relations, operators which are not the highest spin states can pair with components of the current multiplet, forming a long multiplet. Said differently, in the chiral ring, the operators appearing on the righthand side of equation (2.15) are automatically set to zero (since 
they appear as $\bar{D}^{2}$ of something else), and do not parameterize vacua of the deformed theory. This leaves us with just the highest spin states, namely $\mathcal{O}_{j, j}$ for the various spin $j$ representations. Indeed, all other mesons with $\mathcal{O}_{j, s}$ for $s<j$ can be expressed in terms of the $\mathcal{O}_{j, j}$ using the field equations $[16,17,52,53]$.

In particular, we see that any further deformations of the nilpotent orbit, namely a candidate flow from theory $\mathcal{T}_{i}$ to a theory $\mathcal{T}_{i+1}$, will involve precisely these directions. Provided no such operators decouple as we flow from the UV to the IR, this shows that the directed graph defined by the Hasse diagram is also a network of RG flows. The caveat to this statement is that it could indeed happen that some operators decouple as we flow from the UV to the IR. Indeed, as we will shortly explain, for a given $\mathfrak{s u}(2)$ representation, the highest spin states have lowest scaling dimension.

To study this and related issues in more detail, it is of course helpful to have an explicit example where the underlying theory is described by a Lagrangian. In subsequent sections we will present a more general analysis which does not rely on the existence of a Lagrangian.

\subsection{Illustrative Lagrangian example}

We now illustrate some of the above considerations for a UV $\mathcal{N}=2$ SCFT which has a Lagrangian description. Most of the other examples we consider do not admit a convenient presentation of this sort, and so we will instead need to rely on more general abstract considerations.

The example we consider is $\mathcal{N}=2 \mathrm{SU}(2)$ gauge theory with four flavors in the fundamental representation. Some nilpotent mass deformations for this theory were considered previously in [9], so we refer the interested reader there for additional background. Our main interest here will be to characterize every possible nilpotent orbit of the parent $\mathfrak{s o}(8)$ flavor symmetry algebra, and to discuss the explicit structure of the broken symmetry generators.

From the definition of the theory, there is a manifest $\mathfrak{s u}(4)$ flavor symmetry which rotates the fields. In $\mathcal{N}=1$ language, we specify four chiral superfields $q$ in the $(\mathbf{2}, \mathbf{4})$ of $\mathfrak{s u}(2)_{\text {gauge }} \times \mathfrak{s u}(4)_{\text {flav }}$, and four chiral superfields $\widetilde{q}$ in the $(\mathbf{2}, \overline{\mathbf{4}})$ of $\mathfrak{s u}(2)_{\text {gauge }} \times \mathfrak{s u}(4)_{\text {flav }}$. There is also a coupling to the adjoint valued chiral superfield associated with the $\mathfrak{s u}(2)_{\text {gauge }}$ $\mathcal{N}=2$ vector multiplet:

$$
W_{\mathcal{N}=2}=\sqrt{2} \widetilde{q}_{\bar{f}} \varphi q^{f},
$$

where the sum on $f=1, \ldots, 4$ runs over the flavors of the model, and we suppress $\mathfrak{s u}(2)_{\text {gauge }}$ indices. This presentation allows us to explicitly track nilpotent mass deformations associated with the $\mathfrak{s u}(4)$ symmetry algebra, as in reference [9].

Though convenient, this presentation obscures the fact that there is actually an $\mathfrak{s o}(8)$ flavor symmetry. We can assemble the $q$ and $\widetilde{q}$ into an eight-dimensional representation of $\mathrm{SO}(8)$, and instead treat our field content as a half hypermultiplet transforming in the $\left(\mathbf{2}, \boldsymbol{8}_{s}\right)$ of $\mathfrak{s u}(2)_{\text {gauge }} \times \mathfrak{s o}(8)_{\text {flav }}$. Labelling the associated holomorphic chiral superfield by $Q^{i}$ with $i=1, \ldots, 8$, we introduce a conjugate spinor of $\mathrm{SO}(8) Q_{i}^{c}$ which canonically pairs with this field so that the superpotential can then be written as:

$$
W_{\mathcal{N}=2}=\sqrt{2} Q_{i}^{c} \varphi Q^{i}
$$




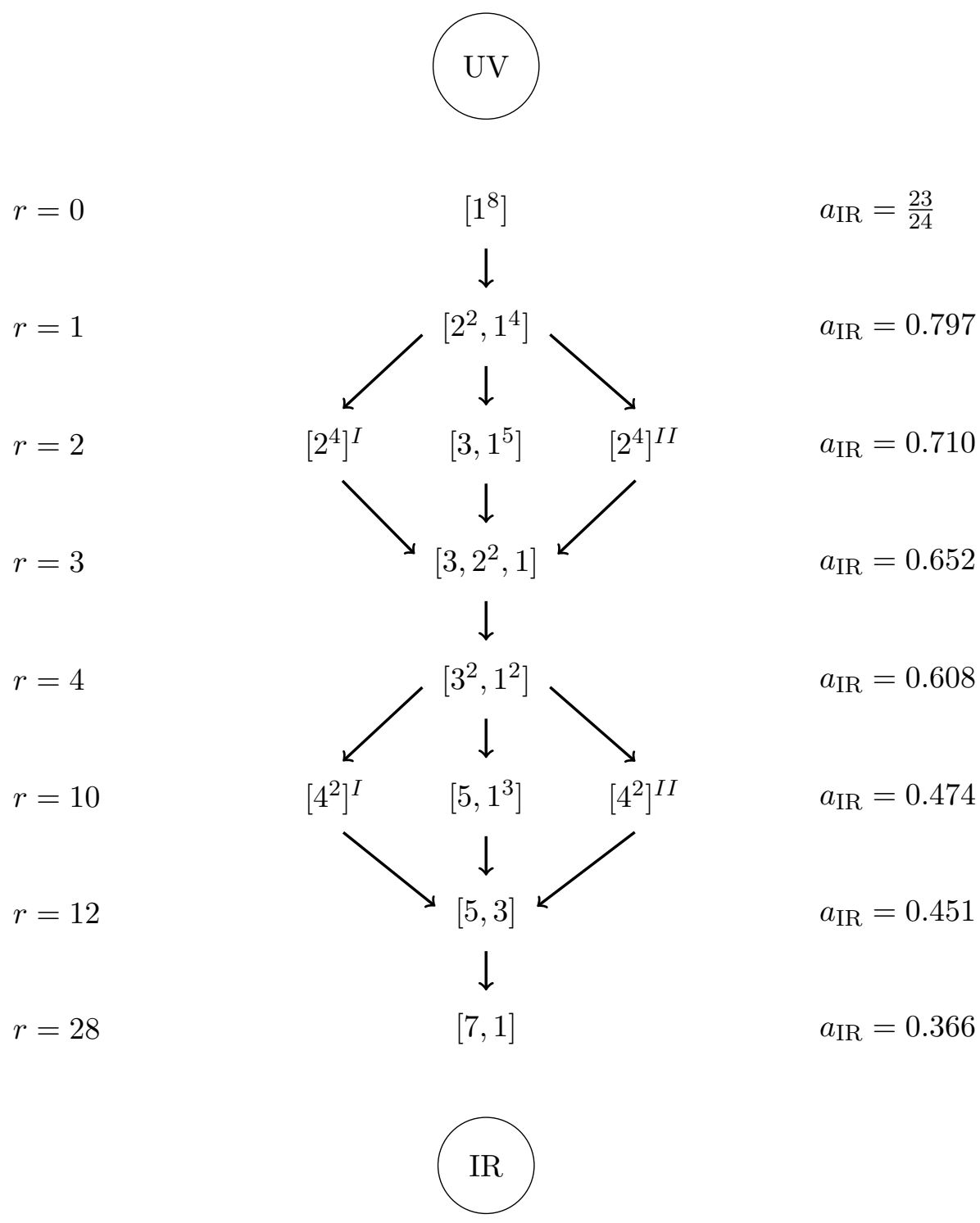

Figure 3. The network of RG flows induced by nilpotent plain mass deformations for $\mathcal{N}=2$ Super Yang-Mills with $\mathrm{SU}(2)$ gauge group and four flavors. This theory has an $\mathrm{SO}(8)$ flavor symmetry in the UV. This network is identical to the Hasse diagram of the Lie algebra $\mathfrak{s o}(8)$. The parameter $r=2 \operatorname{Tr}_{\mathfrak{s o}(8)}\left(T_{3} T_{3}\right)$ is the embedding index for the homomorphism $\mathfrak{s u}(2) \rightarrow \mathfrak{s o}(8)$ defined by a nilpotent orbit. The value of the conformal anomaly $a_{\mathrm{IR}}$ decreases, as expected. These flows are determined using the method described in sections 3 and 4 .

where again, we suppress the $\mathfrak{s u}(2)$ gauge indices. The associated mesons can be written as:

$$
\mathcal{O}^{A}=\left(\rho^{A}\right)_{j}^{i} Q_{i}^{c} Q^{j},
$$

with $\rho^{A}$ the explicit matrix representatives acting on the $\mathbf{8}_{s}$, and $A$ an adjoint index of $\mathrm{SO}(8)$. In this language, nilpotent mass deformations can be viewed as specific choices for the $\rho^{A}$ (upon complexification of the flavor symmetry algebra).

Figure 3 illustrates the resulting network of nilpotent orbits and RG flows in this specific case. We also display the value of $a_{\mathrm{IR}}$ as we pass from the UV to the IR. The 
specific method used to calculate the IR R-charges is essentially the same as in reference [9], and we will discuss it in greater detail in sections 3 and 4 .

Another important aspect of this example is that we can also explicitly track the structure of the broken symmetry currents. To do so, we observe that the Lagrangian density for the $\mathrm{SO}(8)$ theory is, in $\mathcal{N}=1$ language, given by:

$$
\mathcal{L}_{\mathcal{N}=2}=\mathcal{L}_{\text {gauge }}+\int d^{2} \theta d^{2} \bar{\theta} Q_{i}^{\dagger} e^{V} Q^{i}+\int d^{2} \theta W_{\mathcal{N}=2}+\text { h.c. },
$$

with $V$ the $\mathrm{SU}(2) \mathcal{N}=1$ vector multiplet. Here, $\mathcal{L}_{\text {gauge }}$ includes the remaining contributions to the $\mathcal{N}=2$ vector multiplet, namely the kinetic terms for the vector multiplet and adjoint valued chiral superfield.

By varying the action with respect to $Q^{i}$, we obtain the following equation of motion:

$$
-\frac{1}{4} \bar{D}^{2} Q_{i}^{\dagger} e^{V}+2 \sqrt{2}\left(Q^{c}\right)_{i} \varphi=0
$$

For the theory with no mass deformations, we have the on-shell F-term constraint:

$$
\left(Q^{c}\right)_{i} \varphi=0
$$

Using the on shell equations of motion, we observe that the flavor current in the UV:

$$
\mathcal{J}_{A}=\left(\rho_{A}\right)^{j}{ }_{i}\left(Q^{c}\right)_{j}^{\dagger} e^{V} Q^{i},
$$

is actually conserved, namely $\bar{D}^{2} \mathcal{J}_{A}=0$.

Next, we add the superpotential deformation:

$$
W_{D}=m^{j}{ }_{i}\left(Q^{c}\right)_{j} Q^{i}
$$

The current $\mathcal{J}_{A}$ is no longer conserved, because of this explicit breaking term. To see what happens, consider following the Noether procedure with flavor transformation:

$$
\delta_{\text {flav }} Q^{i}=\epsilon_{A}\left(\rho^{A}\right)^{i}{ }_{j} Q^{j} .
$$

This yields:

$$
-\frac{1}{4} \bar{D}^{2} \mathcal{J}_{A}=\left(Q^{c}\right)_{i} m^{i}{ }_{j}\left(\rho_{A}\right)^{j}{ }_{l} Q^{l} .
$$

$m_{j}^{i}$ is the raising operator of the $\mathfrak{s u}(2)_{D}$ subalgebra and expressing the adjoint index $A$ in terms of spin $j$ and $T_{3}$ eigenvalue results exactly in equation (2.15). As already mentioned, an analogous procedure also works for non-Lagrangian theories (see e.g. [16, 17, 52]).

\section{$3 \quad$ Inherited infrared symmetries}

In this section we turn to an analysis of the $4 \mathrm{D} \mathcal{N}=1$ fixed points generated by nilpotent mass deformations, focussing on the structure of the symmetries inherited from the original UV $\mathcal{N}=2$ SCFT. Our aim will be to understand both the structure of the infrared 
R-symmetry, as well as global symmetries preserved by a nilpotent mass deformation. Additionally, we compute the anomalies associated with these symmetries.

One technical assumption we make in this section is that there are no emergent abelian symmetries. When emergent symmetries are present, as necessarily occurs when some operators decouple, it is necessary to track which operators have dimension coming close to the unitarity bound. This requires a more case by case treatment of the nilpotent deformation in question, and is best handled by way of explicit cases.

We begin by treating the case of plain mass deformations and then turn to the case of flipper field deformations. After this, we show that under mild assumptions on the values of $a_{\mathrm{UV}}$ and $c_{\mathrm{UV}}$ that various numerical quantities are strictly monotonic along directed paths through the Hasse diagram of nilpotent orbits.

\subsection{Plain mass deformations}

Suppose, then, that we introduce a nilpotent mass deformation of a $4 \mathrm{D} \mathcal{N}=2 \mathrm{SCFT}$. This initiates an explicit breaking pattern of the $\mathrm{SU}(2) \times \mathrm{U}(1)$ R-symmetry of the UV theory, as well as well as the flavor symmetries $\mathfrak{g}_{\mathrm{UV}}$. By definition, there is a generator $T_{3}$ in the Cartan subalgebra such that the operator $\operatorname{Tr}_{\mathfrak{g}_{\mathrm{UV}}}\left(\mu \cdot \mathcal{O}_{\text {adj }}\right)$ has $T_{3}$ charge -1 . What this means is that a linear combination of $T_{3}$ and $J_{\mathcal{N}=2}$ will remain unbroken along the entire flow to the infrared.

In addition to these symmetries, there are of course all the generators of $\mathfrak{g}_{\text {UV }}$ which commute with our nilpotent orbit. This defines another flavor symmetry algebra $\mathfrak{g}_{\mathrm{IR}}$ which may also include various abelian symmetry factors.

Assuming that we indeed flow to a new fixed point in the infrared with $\mathcal{N}=1$ supersymmetry, the infrared R-symmetry will be a linear combination of all available abelian symmetries:

$$
R_{\mathrm{IR}}=R_{\mathrm{UV}}+t_{J} J_{\mathcal{N}=2}-t T_{3}+t_{\mathrm{other}} T_{\mathrm{other}},
$$

where $T_{\text {other }}$ is shorthand for all other abelian symmetries inherited from the UV.

Now, for our plain mass deformation to be a relevant perturbation, it follows that the IR R-charge of this operator deformation is fixed to be +2 . Since $\operatorname{Tr}_{\mathfrak{g}_{\text {flav }}}\left(\mu \cdot \mathcal{O}_{\text {adj }}\right)$ has charges $R_{\mathrm{UV}}=+4 / 3, J_{\mathcal{N}=2}=-2, T_{3}=-1$ and is neutral under $T_{\text {other }}$, we learn that the IR R-symmetry is actually constrained to be:

$$
R_{\mathrm{IR}}=R_{\mathrm{UV}}+\left(\frac{t}{2}-\frac{1}{3}\right) J_{\mathcal{N}=2}-t T_{3}+t_{\text {other }} T_{\text {other }},
$$

where to fix the remaining parameters $t$ and $t_{\text {other }}$, we must resort to a-maximization [25], namely we calculate the trial value of the conformal anomaly $a_{\text {trial }}\left(t, t_{\text {other }}\right)$ as a function of $t$ and $t_{\text {other: }}$ :

$$
a_{\text {trial }}\left(t, t_{\text {other }}\right)=\frac{3}{32}\left(3 \operatorname{Tr} R_{\mathrm{IR}}^{3}\left(t, t_{\text {other }}\right)-\operatorname{Tr} R_{\mathrm{IR}}\left(t, t_{\text {other }}\right)\right),
$$

and find the local maximum with respect to these parameters. 
Since we are assuming the absence of emergent symmetries in the infrared, we can use anomaly matching to express various IR quantities in terms of UV data. In particular, we shall have need to reference the anomalies:

$$
\begin{aligned}
a_{\mathrm{UV}} & =\frac{3}{32}\left(3 \operatorname{Tr} R_{\mathrm{UV}}^{3}-\operatorname{Tr} R_{\mathrm{UV}}\right) \\
c_{\mathrm{UV}} & =\frac{1}{32}\left(9 \operatorname{Tr} R_{\mathrm{UV}}^{3}-5 \operatorname{Tr} R_{\mathrm{UV}}\right) \\
k_{\mathrm{UV}} \times \delta^{A B} & =-6 \operatorname{Tr}\left(R_{\mathrm{UV}} J_{\text {flav }}^{A} J_{\text {flav }}^{B}\right),
\end{aligned}
$$

in the obvious notation.

Let us first establish that $t_{\text {other }}$ actually vanishes. To this end, we note that since we have assumed below line (2.1) that the anomalies involving the UV flavor symmetries always involve precisely two insertions of the same flavor symmetry, ${ }^{3}$ the only way for $t_{\text {other }}$ to make an appearance in $a_{\text {trial }}$ is through a mixed anomaly with a symmetry generator of the $\mathrm{SU}(2) \times \mathrm{U}(1)$ R-symmetry of the $\mathcal{N}=2 \mathrm{SCFT}$. Since the dependence on $t_{\text {other }}$ has only quadratic dependence, the local maximum necessarily has $t_{\text {other }}=0$. Hence, the infrared R-symmetry is actually given by the linear combination:

$$
R_{\mathrm{IR}}=R_{\mathrm{UV}}+\left(\frac{t}{2}-\frac{1}{3}\right) J_{\mathcal{N}=2}-t T_{3},
$$

with $t$ to be fixed by a-maximization.

This analysis was already carried out in reference [9] for a specific class of deformations, but the generalization to our case follows formally the same steps. The only change is that now, we need to pay attention to the appearance of possibly multiple UV symmetry factors in:

$$
\mathfrak{g}_{\mathrm{UV}}=\mathfrak{g}_{\mathrm{UV}}^{(1)} \times \ldots \times \mathfrak{g}_{\mathrm{UV}}^{(n)},
$$

so we need to label the $R F F$ anomaly for each such factor:

$$
\operatorname{Tr}\left(R_{\mathrm{UV}} J_{A_{i}}^{(i)} J_{B_{i}}^{(i)}\right)=-\frac{k_{\mathrm{UV}}^{(i)}}{6} \delta_{A_{i} B_{i}} .
$$

Since we can decompose our $T_{3}$ generator as a direct sum for each simple factor:

$$
T_{3}=T_{3}^{(1)} \oplus \ldots \oplus T_{3}^{(n)} .
$$

The value of $a_{\text {trial }}(t)$ is given by:

$$
\begin{aligned}
a_{\text {trial }}(t)=\frac{3}{32}[ & \left(36 a_{\mathrm{UV}}-27 c_{\mathrm{UV}}-\frac{9}{4} \sum_{i=1}^{n} k_{\mathrm{UV}}^{(i)} r^{(i)}\right) t^{3} \\
& \left.+\left(-72 a_{\mathrm{UV}}+36 c_{\mathrm{UV}}\right) t^{2}+\left(48 a_{\mathrm{UV}}-12 c_{\mathrm{UV}}\right) t\right]
\end{aligned}
$$

\footnotetext{
${ }^{3}$ Indeed, recall that the "other" in $t_{\text {other }}$ is shorthand for labelling possibly multiple abelian symmetry factors. This means there could be mixed terms between these factors. If all these abelian factors descend from a non-abelian symmetry, such mixed anomalies automatically vanish, but it could a priori still be present for abelian symmetries inherited from the UV theory. This is the main reason the assumption below line (2.1) is required.
} 
where in obtaining this formula we have used the structure of anomalies as dictated by the $\mathrm{UV} \mathcal{N}=2$ theory. Here, $r^{(i)}$ refers to the embedding index for the generator $T_{3}^{(i)}$ in $\mathfrak{g}_{\mathrm{UV}}^{(i)}$ :

$$
r^{(i)} \equiv 2 \operatorname{Tr}_{\mathfrak{g}_{\mathrm{UV}}^{(i)}}\left(T_{3}^{(i)} T_{3}^{(i)}\right)
$$

see appendix A for details.

The local maximum of $a_{\text {trial }}(t)$ is then given by the critical point:

$$
t_{*}=\frac{4}{3} \times \frac{8 a_{\mathrm{UV}}-4 c_{\mathrm{UV}}-\sqrt{4 c_{\mathrm{UV}}^{2}+\left(4 a_{\mathrm{UV}}-c_{\mathrm{UV}}\right) \sum_{i=1}^{n} k_{\mathrm{UV}}^{(i)} r^{(i)}}}{16 a_{\mathrm{UV}}-12 c_{\mathrm{UV}}-\sum_{i=1}^{n} k_{\mathrm{UV}}^{(i)} r^{(i)}} .
$$

With this in hand, we can evaluate the anomalies of our candidate infrared fixed point. In the case of the flavor symmetry anomalies, the structure depends on the remaining flavor symmetry generators associated with each semi-simple factor, and we denote these unbroken symmetry currents by $J_{A_{i}}^{(i)}$. In terms of the parameter $t_{*}$, the IR values of these anomalies are:

$$
\begin{aligned}
a_{\mathrm{IR}}=\frac{3}{32}[ & \left(36 a_{\mathrm{UV}}-27 c_{\mathrm{UV}}-\frac{9}{4} \sum_{i=1}^{n} k_{\mathrm{UV}}^{(i)} r^{(i)}\right) t_{*}^{3} \\
& \left.+\left(-72 a_{\mathrm{UV}}+36 c_{\mathrm{UV}}\right) t_{*}^{2}+\left(48 a_{\mathrm{UV}}-12 c_{\mathrm{UV}}\right) t_{*}\right] \\
c_{\mathrm{IR}}=\frac{1}{32}[ & \left(108 a_{\mathrm{UV}}-81 c_{\mathrm{UV}}-\frac{27}{4} \sum_{i=1}^{n} k_{\mathrm{UV}}^{(i)} r^{(i)}\right) t_{*}^{3} \\
& \left.+\left(-216 a_{\mathrm{UV}}+108 c_{\mathrm{UV}}\right) t_{*}^{2}+\left(96 a_{\mathrm{UV}}+12 c_{\mathrm{UV}}\right) t_{*}\right]
\end{aligned}
$$

and:

$$
K_{\mathrm{IR}}^{(i)}=\frac{3}{2} k_{\mathrm{UV}}^{(i)} \times t_{*}
$$

In the above, we have introduced the anomaly coefficient $K_{\mathrm{IR}}^{(i)}$ :

$$
\operatorname{Tr}\left(R_{\mathrm{IR}} J_{A_{i}}^{(i)} J_{B_{i}}^{(i)}\right)=-\frac{K_{\mathrm{IR}}^{(i)}}{6} \delta_{A_{i} B_{i}}
$$

where we take the same normalization of all Lie algebra generators as inherited from the parent UV symmetry. In a given simple factor in the IR, there could be several subalgebras:

$$
\mathfrak{h}_{1}^{(i)} \times \ldots \times \mathfrak{h}_{m_{i}}^{(i)} \subset \mathfrak{g}_{\mathrm{IR}}^{(i)} \subset \mathfrak{g}_{\mathrm{UV}}^{(i)},
$$

each with a different embedding index. We can of course take generators normalized with respect to these unbroken flavor symmetries to define the more standard quantity via the embedding index:

$$
k_{l_{i}, I R}^{(i)}=\operatorname{Ind}\left(\mathfrak{h}_{l_{i}}^{(i)} \rightarrow \mathfrak{g}_{\mathrm{UV}}^{(i)}\right) \times K_{\mathrm{IR}}^{(i)} .
$$

The physically more meaningful quantity is $k_{\mathrm{IR}}^{(i)}$, though it is often more straightforward to evaluate $K_{\mathrm{IR}}^{(i)}$. 


\subsubsection{Operator scaling dimensions}

Having determined the infrared R-symmetry, we can now extract the scaling dimensions for a number of operators. It is helpful to organize this analysis according to the representation content of the subalgebra $\mathfrak{g}_{\mathrm{IR}} \times \mathfrak{s u}(2)_{D}$, where $\mathfrak{s u}(2)_{D}$ is the subalgebra implicitly defined by a choice of nilpotent orbit. For example, since the mesons transform in the adjoint representation of $\mathfrak{g}_{U V}$, there is a corresponding decomposition into representations:

$$
\begin{aligned}
\mathfrak{g}_{\mathrm{UV}} & \supset \mathfrak{g}_{\mathrm{IR}} \times \mathfrak{s u}(2)_{D} \\
\operatorname{adj}\left(\mathfrak{g}_{\mathrm{UV}}\right) & \rightarrow \bigoplus_{a}\left(R_{(a)}, j_{(a)}\right),
\end{aligned}
$$

where on the righthand side we implicitly sum over irreducible representations of $\mathfrak{g}_{\mathrm{IR}} \times$ $\mathfrak{s u}(2)_{D}$ which appear in the decomposition of the adjoint. More generally, given operators in some representation of $\mathfrak{g}_{\mathrm{UV}}$, we can always decompose into irreducible representations of $\mathfrak{g}_{\mathrm{IR}} \times \mathfrak{s u}(2)_{D}$.

Supposing then that we have a UV operator transforming in a spin $j$ representation of $\mathfrak{s u}(2)_{D}$, we get operators of $T_{3}$ charge $j, j-1, \ldots,-j$, and we can calculate their scaling dimension in the IR theory using our infrared R-symmetry:

$$
\Delta_{\mathrm{IR}}=\frac{3}{2}\left(R_{\mathrm{UV}}+\left(\frac{t_{*}}{2}-\frac{1}{3}\right) J_{\mathcal{N}=2}-t_{*} T_{3}\right) .
$$

In the specific case of a Coulomb branch scalar $Z$, we know that since it has vanishing $I_{3}$ charge, we have $3 R_{\mathrm{UV}}(Z)=J_{\mathcal{N}=2}(Z)$, and $T_{3}(Z)=0$ (as it is neutral under all of $\mathfrak{g}_{\mathrm{UV}}$ ), so we immediately obtain:

$$
\Delta_{\mathrm{IR}}(Z)=\frac{3}{2} t_{*} \times \Delta_{\mathrm{UV}}(Z) .
$$

In the case of a mesonic operator $\mathcal{O}_{j, s}$ transforming in a spin $j$ representation of $\mathfrak{s u}(2)_{D}$, with $T_{3}$ charge $s$, the scaling dimension in the IR is:

$$
\Delta_{\mathrm{IR}}\left(\mathcal{O}_{j, s}\right)=3-\frac{3}{2} t_{*}(1+s) .
$$

\subsubsection{Monotonicity}

With these results in place, we now show that various numerical quantities are indeed monotonic as we proceed to larger orbits in the nilpotent cone. We will also establish this numerically by "brute force" when we turn to an analysis of explicit $\mathcal{N}=2$ theories.

To begin, we recall from reference $[54,55]$ that there is the Hofman-Maldacena bound on the ratio $a_{\mathrm{UV}} / c_{\mathrm{UV}}$ for any $\mathcal{N}=2 \mathrm{SCFT}$ :

$$
\frac{1}{2} \leq \frac{a_{\mathrm{UV}}}{c_{\mathrm{UV}}} \leq \frac{5}{4}
$$

We now use this general bound to establish some monotonicity results for nilpotent mass deformations. 
Now, as we proceed to larger orbits, the size of the corresponding embedding indices necessarily increases. Introducing the parameter:

$$
\mathcal{K} \equiv \sum_{i=1}^{n} k_{\mathrm{UV}}^{(i)} r^{(i)},
$$

we observe that this quantity always increases as we proceed down a directed path in the Hasse diagram. To establish various monotonicity results, it thus suffices to evaluate their response as we vary $\mathcal{K}$.

First of all, we can consider the parameter $t_{*}$ given by equation (3.13), treated as a function of $\mathcal{K}$. If we introduce the Hofman-Maldacena bounds, as well as the constraints from unitarity $a_{\mathrm{UV}}, c_{\mathrm{UV}}, k_{\mathrm{UV}}^{(i)}>0$, we immediately find (as can be checked explicitly using Mathematica) that the derivative:

$$
\frac{\partial t_{*}}{\partial \mathcal{K}}<0,
$$

so in particular, $t_{*}$ always decreases along a flow. Moreover, since the Coulomb branch operators are all proportional to $t_{*}$, we also learn that these dimensions are also always strictly decreasing.

One can also perform a similar analysis for the parameter $a_{\mathrm{IR}}$ as a function of $\mathcal{K}$. In addition to the numerical bounds already introduced, we also require $t_{*}>0$, which in turn requires $16 a_{\mathrm{UV}}-12 c_{\mathrm{UV}}-\mathcal{K}>0$. Curiously enough, we find that in order for this quantity to decrease monotonically, we need to impose a slightly stronger condition than that of line (3.25) for the lower bound:

$$
\frac{3}{4} \leq \frac{a_{\mathrm{UV}}}{c_{\mathrm{UV}}} \leq \frac{5}{4}
$$

The most conservative interpretation of this sharper requirement is that as we pass to larger orbits, we should expect some operators to decouple, in which case the expressions used for $t_{*}$ and $a_{\mathrm{IR}}$ would need to be modified anyway. We will indeed see examples of this type, though we hasten to add that in the explicit models we consider, the sharper condition of line (3.28) is actually satisfied.

\subsection{Flipper field deformations}

Having dealt with the case of plain mass deformations, we now turn to flipper field deformations of an $\mathcal{N}=2 \mathrm{SCFT}$. Recall that this involves promoting the mass parameters of the $\mathcal{N}=2$ theory to an adjoint valued chiral superfield, and switching on a background vev:

$$
\delta W=\operatorname{Tr}_{\mathfrak{g}_{\text {flav }}}\left(\left(m_{\mathrm{adj}}+M_{\mathrm{adj}}\right) \cdot \mathcal{O}_{\mathrm{adj}}\right) .
$$

Again, we confine our analysis to the case where this vev is a nilpotent mass deformation.

Since we are activating a breaking pattern which is identical to the case of the plain mass deformation, much of the analysis of the previous section will carry over unchanged. The primary issue is that now, we need to track the additional modifications to the infrared R-symmetry which come from having these additional fields transforming in the adjoint representation. 
From the perspective of the UV theory, we have two decoupled SCFTs, namely the original $\mathcal{N}=2$ fixed point, and a decoupled free chiral multiplet. Consequently, there is a $\mathrm{U}(1)$ flavor symmetry with generator $T_{\text {flip }}$ which acts on each flipper field, so that it has charge +1 . The trial infrared R-symmetry is then a general linear combination of the form:

$$
R_{\mathrm{IR}}^{\text {flip }}(t)=R_{\mathrm{IR}}^{\text {plain }}(t)+t_{\text {flip }} T_{\text {flip }}
$$

where we have also left implicit the sum over all flippers. Here, the trial infrared Rsymmetry in the case of a plain mass deformation is:

$$
R_{\mathrm{IR}}^{\text {plain }}(t)=R_{\mathrm{UV}}+\left(\frac{t}{2}-\frac{1}{3}\right) J_{\mathcal{N}=2}-t T_{3}
$$

Now, upon decomposing into representations of $\mathfrak{s u}(2)_{D}$, we see that all flipper fields will deform the theory via operators such as $M_{j,-s} \mathcal{O}_{j, s}$. If we first activate the plain mass deformation, and then couple to the flipper fields, we see that since the operators $\mathcal{O}_{j, j}$ with the highest spin have the lowest scaling dimension, then these are the operators which actually drive a new flow $[16,17]$. For this to be so, we require a constraint on the infrared R-charge assignments (see e.g. [12, 53]):

$$
R_{\mathrm{IR}}\left(M_{j,-j}\right)+R_{\mathrm{IR}}\left(\mathcal{O}_{j, j}\right)=2
$$

so the new trial IR R-symmetry is:

$$
R_{\mathrm{IR}}^{\mathrm{flip}}(t)=R_{\mathrm{IR}}^{\text {plain }}(t)+\left(t-\frac{2}{3}\right) T_{\text {flip }}
$$

We can also calculate the new trial $a_{\text {trial }}^{\text {flip }}(t)$ by breaking up the trace over states into those coming from the original $\mathcal{N}=2$ theory, and those coming from the flipper fields which actually participate in the flow. Doing so, we get:

$$
\left.a_{\text {trial }}^{\text {flip }}(t)=a_{\text {trial }}^{\text {plain }}(t)+\sum_{j_{(a)}}\left[\frac{3}{32}\left(3\left(R_{\mathrm{IR}}^{\mathrm{flip}}\left(M_{j_{(a)},-j_{(a)}}\right)-1\right)^{3}-\left(R_{\mathrm{IR}}^{\mathrm{flip}}\left(M_{j_{(a)},-j_{(a)}}\right)-1\right)\right)\right)\right],
$$

where in the first term, $a_{\text {trial }}^{\text {plain }}(t)$ is the same quantity as in line (3.11), and in the second set of terms, we sum over all highest spin states which appear in the branching rules for the $\mathfrak{s u}(2)_{D}$ subalgebra. The R-charge for each such flipper field is evaluated with respect to the original R-symmetry of the plain mass deformation case, namely:

$$
R_{\mathrm{IR}}^{\text {plain }}\left(M_{j_{(a)},-j_{(a)}}\right)=\frac{2}{3}+j_{(a)} \times t .
$$

Maximizing over the parameter $t$ appearing in $a_{\text {trial }}^{\text {flip }}(t)$, we again obtain the infrared R-symmetry, and can read off the scaling dimensions of operators, much as before. By a similar token, we can also read off the new value of the conformal anomaly $c_{\mathrm{IR}}^{\text {flip }}$. Collecting these expressions here, we have [56]:

$$
\begin{aligned}
& \left.a_{\mathrm{IR}}^{\mathrm{flip}}=a_{\mathrm{IR}}^{\text {plain }}\left(t_{*}\right)+\sum_{j_{(a)}}\left[\frac{3}{32}\left(3\left(R_{\mathrm{IR}}^{\mathrm{flip}}\left(M_{j_{(a)},-j_{(a)}}\right)-1\right)^{3}-\left(R_{\mathrm{IR}}^{\mathrm{flip}}\left(M_{j_{(a)},-j_{(a)}}\right)-1\right)\right)\right)\right] \\
& \left.c_{\mathrm{IR}}^{\mathrm{flip}}=a_{\mathrm{IR}}^{\text {plain }}\left(t_{*}\right)+\sum_{j_{(a)}}\left[\frac{1}{32}\left(9\left(R_{\mathrm{IR}}^{\mathrm{flip}}\left(M_{j_{(a)},-j_{(a)}}\right)-1\right)^{3}-5\left(R_{\mathrm{IR}}^{\mathrm{flip}}\left(M_{j_{(a)},-j_{(a)}}\right)-1\right)\right)\right)\right],
\end{aligned}
$$

in the obvious notation. 
With the infrared R-symmetry in hand, we can also evaluate the new anomalies involving the flavor symmetry. Since the flipper fields also transform in irreducible representations of $\mathfrak{g}_{\mathrm{IR}}$, the IR flavor symmetry, we need to take into account the specific branching rules associated with the decomposition of the adjoint representation. With notation as in line (3.19), we have:

$$
k_{\mathrm{IR}}\left(\mathfrak{h}_{l_{i}}^{(i)}\right)=\operatorname{Ind}\left(\mathfrak{h}_{l_{i}}^{(i)} \rightarrow \mathfrak{g}_{\mathrm{UV}}^{(i)}\right) \times K_{\mathrm{IR}}\left(\mathfrak{g}_{\mathrm{UV}}\right)+6 \sum_{j_{(a)}}\left(1-\left(1-T_{3}\left(M_{j_{(a)},-j_{(a)}}\right)\right) t_{*}\right) \operatorname{Ind}\left(\rho_{a}\left(\mathfrak{h}_{l_{i}}^{(i)}\right)\right) .
$$

Here, $\operatorname{Ind}\left(\rho_{a}\left(\mathfrak{h}_{l}^{(i)}\right)\right)$ indicates the index of the representation associated with a given flipper field for the flavor symmetry algebra $\mathfrak{h}_{l_{i}}^{(i)}$.

Much as in the case of the plain mass deformations, we can read off the scaling dimensions of our operators. The operator scaling dimensions for the Coulomb branch scalars and mesonic operators are basically the same as in lines (3.23) and (3.24) except that now we use a modified value for $t_{*}$ due to the coupling to flipper fields. In the case of the flipper fields, we can read off the scaling dimensions of those that actually participate in a flow via equation (3.32). For those flipper fields which do not actually participate in a flow, we instead have a collection of decoupled free fields. In what follows, we shall ignore these contributions, focussing exclusively on the interacting fixed point.

\section{Emergent symmetries and operator decoupling}

In our analysis so far, we have assumed that there are no emergent symmetries in the infrared. Our aim in this section will be to discuss some general features of when to expect emergent symmetries in the case of nilpotent mass deformations. We turn to specific UV theories in the following sections. Turning the discussion around, the mathematical ordering of nilpotent orbits provides some helpful clues on the nature of these candidate fixed points.

Now, one way such emergent symmetries can show up is when various operators start to decouple. Assuming that a fixed point is really present, if we assume the absence of emergent symmetries and find the pathological behavior that some operator has dimension below the unitarity bound, then it is an indication that this operator has actually decoupled. The minimal procedure of reference [42] prescribes that we introduce an additional U(1) flavor symmetry which only acts on the offending operator. From our starting point of an $\mathcal{N}=2$ theory, the main thing we will be able to check is the scaling dimension of the Coulomb branch and mesonic operators of the UV parent theory.

Another related possibility is that the IR theory actually enhances to an $\mathcal{N}=2$ supersymmetric theory in the infrared. This can occur, for example, in the case of flipper field deformations $[16,17]$, and recently a set of general sufficient conditions for such behavior to occur were proposed in [57]. A necessary (but insufficient) condition to have such an enhancement is that the various anomalies of the IR fixed point all become rational numbers rather than the algebraic numbers present for a more general nilpotent mass deformation. There are however known counter-examples that have rational anomalies but no SUSY enhancement to $\mathcal{N}=2[58]$. 
Our plan in this section will be to setup some general diagnostics for symmetry enhancement in the case of nilpotent mass deformations. First, we consider the decoupling of Coulomb branch operators, and then we turn to the decoupling of mesonic operators. After this we discuss some special cases associated with rational values for the anomalies. Finally, we discuss some preliminary aspects of how the partial ordering implied by a Hasse diagram lines up with the physical RG flows.

\subsection{Decoupling of Coulomb branch operators}

Suppose then, that we perform our initial a-maximization procedure, and, assuming the absence of any emergent U(1)'s, we calculate the scaling dimension of a Coulomb branch operator $Z$. According to our general formula from line (3.23), we have:

$$
\Delta_{\mathrm{IR}}(Z)=\frac{3}{2} t_{*} \times \Delta_{\mathrm{UV}}(Z)
$$

If this yields a value less than one, but we still expect the presence of an IR fixed point, this is a strong indication that this operator has actually decoupled (and so has dimension exactly one). By inspection of our expression for the parameter $t_{*}$ we see that this occurs whenever the embedding index becomes sufficiently large.

Assuming this is the only operator to decouple, it is also straightforward to calculate the new infrared R-symmetry. Following appendix B of [43], we have:

$$
\begin{aligned}
a_{\mathrm{IR}}^{\text {new }}(t)=a_{\mathrm{IR}}^{\text {old }}(t)+\frac{3}{32}[ & \left(3\left(R_{\text {old }}(Z)+t_{Z}-1\right)^{3}-3\left(R_{\text {old }}(Z)-1\right)^{3}\right) \\
& \left.-\left(\left(R_{\text {old }}(Z)+t_{Z}-1\right)-\left(R_{\text {old }}(Z)-1\right)\right)\right]
\end{aligned}
$$

for $a$ in the IR. Here, $t_{Z}$ denotes the charge of $Z$ under the emergent U(1) which only acts on this operator. Performing $a$-maximization with respect to $t_{Z}$ then yields

$$
R_{\text {new }}(Z) \equiv R_{\text {old }}(Z)+t_{Z}=\frac{2}{3} .
$$

At this point, we see that adding the emergent $\mathrm{U}(1)$ indeed corrects the scaling dimension of the offending operator to one, and it decouples. Substituting in this result, along with the fact that $R_{\text {old }}(Z)=t \times \Delta_{\mathrm{UV}}(Z)$ implies

$$
a_{\mathrm{IR}}^{\text {new }}(t)=a_{\mathrm{IR}}^{\text {old }}(t)-\frac{3}{32}\left[3\left(\Delta_{\mathrm{UV}}(Z) t-1\right)^{3}-\left(\Delta_{\mathrm{UV}}(Z) t-1\right)\right]+\frac{1}{48} .
$$

Now, we perform the second part of $a$-maximization by taking the partial derivative of $a_{\mathrm{IR}}^{\text {new }}(t)$ with respect to $t$ and setting it equal to zero. For the new value

$$
\begin{aligned}
t_{*}^{\text {new }}=- & \frac{4}{3\left(48 a_{\mathrm{UV}}-36 c_{\mathrm{UV}}-3 k_{\mathrm{UV}} r-4 \Delta_{\mathrm{UV}}^{3}\right)}\left(-24 a_{\mathrm{UV}}+12 c_{\mathrm{UV}}+3 \Delta_{\mathrm{UV}}^{2}\right. \\
+ & \left\{36 c_{\mathrm{UV}}^{2}+36 a_{\mathrm{UV}} k_{\mathrm{UV}} r-6 k_{\mathrm{UV}} r \Delta_{\mathrm{UV}}+48 a_{\mathrm{UV}}\left(-2+\Delta_{\mathrm{UV}}\right)\left(-1+\Delta_{\mathrm{UV}}\right) \Delta_{\mathrm{UV}}+\Delta_{\mathrm{UV}}^{4}\right. \\
& \left.\left.-3 c_{\mathrm{UV}}\left(3 k_{\mathrm{UV}} r+4 \Delta_{\mathrm{UV}}\left(6+\left(-6+\Delta_{\mathrm{UV}}\right) \Delta_{\mathrm{UV}}\right)\right)\right\}^{1 / 2}\right)
\end{aligned}
$$


we find a maximum of $a_{\mathrm{IR}}^{\text {new }}$. Note that we use the abbreviation $\Delta_{\mathrm{UV}}$ for $\Delta_{\mathrm{UV}}(Z)$ in this equation to increase the brevity. One can check that the second derivative of the trial $a_{\mathrm{IR}}^{\text {new }}(t)$ is indeed negative definite at the critical point, so we do get a local maximum.

Let us summarize the central charges after decoupling the offending operator:

$$
\begin{aligned}
a_{\mathrm{IR}}^{\text {new }} & =a_{\mathrm{IR}}^{\text {old }}\left(t_{*}^{\text {new }}\right)-\frac{3}{32}\left[3\left(\Delta_{\mathrm{UV}}(Z) t_{*}^{\text {new }}-1\right)^{3}-\left(\Delta_{\mathrm{UV}}(Z) t_{*}^{\text {new }}-1\right)\right]+\frac{1}{48} \\
c_{\mathrm{IR}}^{\text {new }} & =c_{\mathrm{IR}}^{\text {old }}\left(t_{*}^{\text {new }}\right)-\frac{1}{32}\left[9\left(\Delta_{\mathrm{UV}}(Z) t_{*}^{\text {new }}-1\right)^{3}-5\left(\Delta_{\mathrm{UV}}(Z) t_{*}^{\text {new }}-1\right)\right]+\frac{1}{24} \\
K_{\mathrm{IR}}^{\text {new }} & =K_{\mathrm{IR}}^{\text {old }}\left(t_{*}^{\text {new }}\right),
\end{aligned}
$$

where $a_{\mathrm{IR}}^{\mathrm{old}}, c_{\mathrm{IR}}^{\text {old }}$, and $K_{\mathrm{IR}}^{\mathrm{old}}$ are the central charges which were computed without the emergent $\mathrm{U}(1)$. We emphasize that $K_{\mathrm{IR}}$ does not receive any additional contributions besides $K_{\mathrm{IR}}^{\text {old }}\left(t_{*}^{\text {new }}\right)$ due to the fact that $Z$ is not charged under the flavor symmetry. Thus, removing the contribution from such operators does not directly affect the flavor central charge, just indirectly by modifying the value of $t_{*}$.

\subsection{Decoupling of mesonic operators}

Let us now turn to the possible decoupling of mesonic operators. When we turn to specific examples, we find that this does not occur for the probe D3-brane theories, but does occur for $4 \mathrm{D}$ conformal matter theories.

We first treat the case of plain mass deformations, and then turn to the case of flipper field deformations. Returning to our general formula for the operator scaling dimensions (in the absence of emergent $\mathrm{U}(1)$ 's), we see from equation (3.24) that the scaling dimension of an operator $\mathcal{O}_{j, s}$ is:

$$
\Delta_{\mathrm{IR}}\left(\mathcal{O}_{j, s}\right)=3-\frac{3}{2} t_{*}(1+s) .
$$

So, the bigger the spin of the operator under the $\mathfrak{s u}(2)_{D}$ subalgebra, the smaller the scaling dimension. This is counteracted to some extent by the decreasing value of $t_{*}$, though in practice, it is still true that as we descend to larger nilpotent orbits, more mesonic operators start to decouple. For a given spin $j$ representation of $\mathfrak{s u}(2)_{D}$, it is hopefully clear that the highest spin state with $s=j$ will have lowest candidate scaling dimension, so if this operator has scaling dimension above the unitarity bound, the remaining operators in the same $\mathfrak{s u}(2)_{D}$ multiplet will also be above the bound.

On the other hand, if the highest spin operator falls below the unitarity bound, we can again posit that it decouples, with a single emergent $\mathrm{U}(1)$ which acts only on this operator. Now, in addition to the highest spin operator $\mathcal{O}_{j, j}$, there are often other values of $s$ in the same multiplet which might also appear to violate the unitarity bound. Note, however, that via our previous discussion of the broken flavor symmetry generators and the relation of equation (2.15):

$$
-\frac{1}{4} \bar{D}^{2} \mathcal{J}_{j, s}=\mathcal{O}_{j, s-1}
$$

we know that components of the flavor current and the mesons pair up in long multiplets. As a result, we again only need to apply our procedure for the "top spin" operators of a given $\mathfrak{s u}(2)_{D}$ multiplet. 
Once again, reference [43] tells us that all we need to do is remove the contribution from the offending operator $\mathcal{O}_{i}$ as follows:

$$
\begin{aligned}
a_{\mathrm{IR}}^{\text {new }}(t)=a_{\mathrm{IR}}^{\text {old }}(t)+\sum_{i} \frac{3}{32}[ & \left(3\left(R_{\text {old }}\left(\mathcal{O}_{i}\right)+t_{\mathcal{O}_{i}}-1\right)^{3}-3\left(R_{\text {old }}\left(\mathcal{O}_{i}\right)-1\right)^{3}\right) \\
& \left.-\left(\left(R_{\text {old }}\left(\mathcal{O}_{i}\right)+t_{\mathcal{O}_{i}}-1\right)-\left(R_{\text {old }}\left(\mathcal{O}_{i}\right)-1\right)\right)\right] .
\end{aligned}
$$

Naively, one would take the index $i$ in this equation to run over all mesons which appear to have dimension below the unitarity bound. However, our discussion of the deformed symmetry current near line (2.15) shows that only the highest spin component of each $\mathfrak{s u}(2)_{D}$ multiplet actually participates in the chiral ring of the IR fixed point.

The procedure of $a$-maximization with respect to $t_{\mathcal{O}_{i}}$ then yields

$$
R_{\text {new }}\left(\mathcal{O}_{i}\right) \equiv R_{\text {old }}\left(\mathcal{O}_{i}\right)+t_{\mathcal{O}_{i}}=\frac{2}{3} .
$$

Again, we see that all bad $\mathcal{O}_{i}$ decouple. The value of $t_{*}$ is determined by $a$-maximization of $a_{\mathrm{IR}}^{\text {new }}(t)$ and the corresponding anomalies are:

$$
\begin{aligned}
& a_{\mathrm{IR}}^{\text {new }}=a_{\mathrm{IR}}^{\mathrm{old}}(t)-\sum_{i} \frac{3}{32}\left[3\left(R_{\text {old }}\left(\mathcal{O}_{i}\right)-1\right)^{3}-\left(R_{\text {old }}\left(\mathcal{O}_{i}\right)-1\right)\right]+\frac{1}{48} \\
& c_{\mathrm{IR}}^{\text {new }}=c_{\mathrm{IR}}^{\text {old }}(t)-\sum_{i} \frac{1}{32}\left[9\left(R_{\text {old }}\left(\mathcal{O}_{i}\right)-1\right)^{3}-5\left(R_{\text {old }}\left(\mathcal{O}_{i}\right)-1\right)\right]+\frac{1}{24} .
\end{aligned}
$$

We can also give a general formula for the new $k_{\mathrm{IR}}\left(\mathfrak{h}_{l_{i}}^{(i)}\right)$ after we decouple all the offending mesons:

$$
k_{\mathrm{IR}}\left(\mathfrak{h}_{l_{i}}^{(i)}\right)=\operatorname{Ind}\left(\mathfrak{h}_{l_{i}}^{(i)} \rightarrow \mathfrak{g}_{\mathrm{UV}}^{(i)}\right) \times K_{\mathrm{IR}}\left(\mathfrak{g}_{\mathrm{UV}}^{(i)}\right)-6 \sum_{a}\left(1-\left(1+T_{3}\left(\mathcal{O}_{a}\right)\right) t_{*}\right) \operatorname{Ind}\left(\rho_{a}\left(\mathfrak{h}_{l_{i}}^{(i)}\right)\right)
$$

where $\operatorname{Ind}\left(\rho_{a}\left(\mathfrak{h}_{l}^{(i)}\right)\right)$ is the index of the irreducible representation under which $\mathcal{O}_{i}$ transforms, and $t_{*}$ is the fixed value of the maximization parameter at the last step when there are no unitarity bound violations anymore.

Consider next the case of mesonic operators which decouple in the flipper field deformations. As noted in [53], when an operator decouples, one can introduce an additional "flipping field" which couples to this field. Doing this is equivalent to the standard procedure of introducing an additional U(1) anyway. Let us see how this works in detail.

With each $M$, there comes an additional U(1) symmetry in the UV theory. Coupling the mesons to the $M$ 's protects them from dropping below the unitarity bound in the IR. From another point of view, the process of removing one of the previously offending $\mathcal{O}$ 's is equivalent to adding a coupling to $M$, as explained in [53]. Compared to the plain mass deformation the new $\mathrm{UV} \mathrm{U}(1)$ is equivalent to the emergent $\mathrm{U}(1)$ that we would have to introduce by hand, once a meson drops below the unitarity bound. Hence, for all flipper field deformations we do not need to worry about any of the mesons decoupling or how it might affect the anomalies. This is automatically being taken care of by the $M$ 's. In fact as explained in [53], the mesons $\mathcal{O}$ are zero in the chiral ring, and therefore there are 
no unitarity violations associated to them. In the following, we describe this intriguing mechanism in more detail from another point of view.

The analysis involves essentially the same equations as already presented in section 3 , which we present here for convenience of the reader. Recall that with flipper field deformations, we have a free chiral superfield $M$ in the adjoint of $\mathfrak{g}_{\mathrm{UV}}$ coupled to $\mathcal{O}_{\text {adj }}$ via $\delta W=\operatorname{Tr}_{\mathfrak{g}_{\mathrm{UV}}}\left(M_{\mathrm{adj}} \cdot \mathcal{O}_{\mathrm{adj}}\right)$, with a background value $\left\langle M_{\mathrm{adj}}\right\rangle=m_{\text {adj }}$ our nilpotent mass term. There is automatically an extra U(1) symmetry for each $M_{j_{(a)},-j_{(a)}}$ in the UV. The first part of the trial IR R-charge is fixed by the plain mass deformation term $\operatorname{Tr}_{\mathfrak{g}_{\mathrm{UV}}}\left(m_{\text {adj }} \cdot \mathcal{O}_{\text {adj }}\right)$. In the UV the $M_{j_{(a)},-j_{(a)}}$ are free multiplets and they are charged under an extra U(1). We call the generator corresponding the this extra $\mathrm{U}(1) T_{\text {flip }}$. The charge of the fluctuation of $M$ is normalized to $T_{\text {flip }}(M)=1$, and nothing else is charged under it. Moreover we know that $T_{3}\left(M_{j_{(a)},-j_{(a)}}\right)=-T_{3}\left(\mathcal{O}_{\left(j_{(a)}, j_{(a)}\right)}\right)=-j_{(a)}$. Now, we have to take this additional symmetry into account while computing the trial IR R-charge

$$
R_{\mathrm{IR}}=R_{\mathrm{UV}}+\left(\frac{t}{2}-\frac{1}{3}\right) J_{\mathcal{N}=2}-t T_{3}+t_{\text {flip }} T_{\text {flip }}
$$

Applying this relation to the superpotential deformation $\delta W$, we find

$$
R_{\mathrm{IR}}^{\text {new }}(\delta W)=R_{\mathrm{IR}}^{\mathrm{old}}\left(\mathcal{O}_{j_{(a)}, j_{(a)}}\right)+t_{\text {flip }}+\frac{2}{3}-t T_{3}\left(M_{\left(j_{(a)},-j_{(a)}\right)}\right)
$$

So, we have:

$$
\begin{aligned}
R_{\mathrm{IR}}^{\mathrm{old}}(\mathcal{O})+t_{\text {flip }}+\frac{2}{3}-t T_{3}\left(M_{j_{(a)},-j_{(a)}}\right) & =2 \\
\Longrightarrow t_{\text {flip }}=\frac{4}{3}-R_{\mathrm{IR}}^{\mathrm{old}}(\mathcal{O})+t T_{3}\left(M_{j_{(a)},-j_{(a)}}\right) & =t-\frac{2}{3}
\end{aligned}
$$

This implies an additional contribution to $a_{\mathrm{IR}}=a_{\mathrm{IR}}^{\text {old }}+\delta a_{\mathrm{IR}}$ as follows:

$$
\begin{aligned}
\delta a_{\mathrm{IR}} & =\frac{3}{32}\left[3\left(t_{\text {flip }} T_{\text {flip }}(M)-t_{*} T_{3}(M)+R_{\mathrm{UV}}(M)-1\right)^{3}-\left(t_{\text {flip }} T_{\text {flip }}-t_{*} T_{3}(M)+R_{\mathrm{UV}(M)-1}\right)\right] \\
& =\frac{3}{32}\left[3\left(t_{\text {flip }} T_{\text {flip }}(M)-t_{*} T_{3}(M)\right)^{3}-3\left(t_{\text {flip }} T_{\text {flip }}(M)-t_{*} T_{3}(M)\right)^{2}+\frac{2}{9}\right] \\
& =\frac{3}{32}\left[3\left(\frac{4}{3}-R_{\mathrm{IR}}(\mathcal{O})\right)^{3}-3\left(\frac{4}{3}-R_{\mathrm{IR}}(\mathcal{O})\right)^{2}+\frac{2}{9}\right] \\
& =-\frac{3}{32}\left[3\left(R_{\mathrm{IR}}(\mathcal{O})-1\right)^{3}-\left(R_{\mathrm{IR}}(\mathcal{O})-1\right)\right] .
\end{aligned}
$$

As a result we can see that adding an additional $\mathrm{U}(1)$ through the above coupling is equivalent to removing the contribution from the "bad" operators directly. This is why the flipper fields automatically rescue the mesons whenever they would naively drop below the unitarity bound had this coupling not been there. These additional coupling terms are identical to the ones that we were forced to add whenever one of the mesons dropped below the unitarity bound before adding flipper fields. 
Another quicker approach which builds upon equation (4.19) is to make use of the fact that $R(M)+R(\mathcal{O})=2$ so that we get:

$$
\begin{aligned}
\delta a_{\mathrm{IR}} & =-\frac{3}{32}\left[3\left(2-R_{\mathrm{IR}}(M)-1\right)^{3}-\left(2-R_{\mathrm{IR}}(M)-1\right)\right] \\
& =-\frac{3}{32}\left[3\left(-R_{\mathrm{IR}}(M)+1\right)^{3}-\left(-R_{\mathrm{IR}}(M)+1\right)\right] \\
& =\frac{3}{32}\left[3\left(R_{\mathrm{IR}}(M)-1\right)^{3}-\left(R_{\mathrm{IR}}(M)-1\right)\right] .
\end{aligned}
$$

Therefore, adding directly the contribution from the $M$ 's is equivalent to removing the contribution from the "bad" $\mathcal{O}$ 's. This recovers our expressions for $a_{\mathrm{IR}}$ and $c_{\mathrm{IR}}$ up to the presence of free chiral multiplets that do not couple.

As a result, none of the mesons in the flipper deformed theories can drop below the unitarity bound because they are all automatically rescued by the $M$ 's to which they couple.

\subsection{Rational theories}

One of the interesting features of the "brute force" sweeps we perform in later sections reveals that in some cases, the anomalies are all rational numbers, even though a priori, we should only expect algebraic numbers as per the procedure of a-maximization. We refer to such IR fixed points as rational theories. Clearly, this suggests some additional emergent structure in the infrared, and in some favorable circumstances, this can also be identified with the appearance of enhanced $\mathcal{N}=2$ supersymmetry, as in the case of the Maruyoshi-Song deformations [16, 17]. In the specific examples we consider, we find that this can happen both with and without operators decoupling, and both for plain mass deformations and flipper field deformations, see appendix $\mathrm{C}$ for details.

There has very recently been some progress in understanding some additional sufficient criteria for $\mathcal{N}=2$ enhancements [57]. The main idea in this analysis is that whenever we encounter a flavor singlet operator of the IR theory, we need to be able to interpret as a scalar operator parameterizing a direction of the Coulomb branch. This is not the case in our rational theories, but it is also unclear whether there is any additional supersymmetry enhancement. We leave a full treatment of possible enhancements in these theories for future work.

\subsection{Ordering of RG flows}

As we can see, there is no clean expression that describes $a_{\mathrm{IR}}$ as a function of the embedding index, once we take into account operators that decouple in the IR. One might rightfully worry that $a_{\mathrm{IR}}$ would not necessarily be a simple monotonically decreasing function of $r$ anymore. However, we observe empirically that the RG flows continue to follow the trajectory of paths through the Hasse diagram, even after introducing emergent U(1)'s and flipper field operators. This is explicitly shown in the explicit examples we consider.

We close this section with two important remarks:

1. If no operator drops below the unitarity bound, the theories are guaranteed to follow the flow pattern specified by the Hasse diagrams. 
2. In all of the other cases studied in this paper, even when operators decouple, we still observe that the RG flows respect the partial ordering of nilpotent orbits. So, while the RG flows could have a weaker ordering than the mathematical ordering (if the wrong mesons hit the unitarity bound) we see that they do not appear to violate the partial ordering of nilpotent orbits.

\section{D3-brane probe theories}

In the previous sections we introduced a general procedure for treating nilpotent mass deformations. In this section, we turn to a systematic analysis of all such deformations for the $\mathcal{N}=2$ theories defined by a D3-brane probing a 7-brane with $D_{4}, E_{6}, E_{7}$ or $E_{8}$ flavor symmetry. In what follows we do not include the contribution from the decoupled free hypermultiplet with scalars parameterizing motion of the D3-brane parallel to the 7-brane.

Some examples of nilpotent mass deformations for these theories were analyzed in [9], as well as [16]. In the F-theory interpretation where we wrap the 7 -brane on a surface $\mathcal{S}_{\mathrm{GUT}}$, we have a partially twisted gauge theory with a $(0,1)$-connection and an adjoint valued $(2,0)$ form $\Phi_{(2,0)}[59]$ (see also [60,61]). In terms of the associated F-theory geometry, deformations of $\Phi_{(2,0)}$ with non-vanishing Casimir invariant translate to complex structure deformations of the associated elliptically fibered Calabi-Yau fourfold. The nilpotent case is especially interesting because it is essentially "invisible" to the complex geometry of the model. We can then view the mass parameters $m_{\text {adj }}$ as background values for $\Phi_{(2,0)}[9,10]$, and the particular case of a nilpotent mass deformation defines a T-brane configuration [11, 31-41].

From this perspective, it is also natural to view the flipper field deformation as promoting the zero mode of $\Phi_{(2,0)}$ to a dynamical field. This is actually somewhat subtle in the context of a full F-theory compactification, because making $\Phi_{(2,0)}$ dynamical requires us to wrap the 7-brane on a compact Kähler surface, which also introduces dynamical gauge fields (zero modes from the $(0,1)$ connection can be eliminated by choosing a suitable surface and background vector bundle). However, by introducing a sufficiently large number of additional spectator fields which also interact with this gauge field, we can always take a limit where this gauge theory is infrared free (in contrast to the case typically assumed in decoupling limits from gravity).

In both the case of plain mass deformations as well as its extension to flipper field deformations, we see that the IR fixed points defined by the D3-brane provide additional insight into the structure of T-brane configurations in F-theory.

Let us now turn to an analysis of the fixed points in these theories. Much as in the earlier sections of this paper, it is helpful to split our analysis up into the cases of plain mass deformations and flipper field deformations. We also discuss in detail the special case of rational theories, which suggest additional structure in the IR. This includes all the previous $\mathcal{N}=2$ enhancement theories found in [16], as well as another one which comes about from deformations of the $E_{7}$ Minahan-Nemeschansky theory (see also [62]). 


\begin{tabular}{|c|c|c|c|c|c|c|c|}
\hline$G$ & $H_{0}$ & $H_{1}$ & $H_{2}$ & $D_{4}$ & $E_{6}$ & $E_{7}$ & $E_{8}$ \\
\hline$\Delta_{\mathrm{UV}}(Z)$ & $\frac{6}{5}$ & $\frac{4}{3}$ & $\frac{3}{2}$ & 2 & 3 & 4 & 6 \\
\hline$a_{\mathrm{UV}}$ & $\frac{43}{120}$ & $\frac{11}{24}$ & $\frac{7}{12}$ & $\frac{23}{24}$ & $\frac{41}{24}$ & $\frac{59}{24}$ & $\frac{95}{24}$ \\
\hline$c_{\mathrm{UV}}$ & $\frac{11}{30}$ & $\frac{1}{2}$ & $\frac{2}{3}$ & $\frac{7}{6}$ & $\frac{13}{6}$ & $\frac{19}{6}$ & $\frac{31}{6}$ \\
\hline$k_{\mathrm{UV}}$ & $\frac{12}{5}$ & $\frac{8}{3}$ & 3 & 4 & 6 & 8 & 12 \\
\hline
\end{tabular}

Table 2. Scaling dimensions and anomalies of rank $14 \mathrm{D} \mathcal{N}=2$ SCFTs.

\subsection{Summary of UV $\mathcal{N}=2$ fixed points}

In this section we briefly summarize some aspects of the $\mathcal{N}=2$ theories. We first list the anomalies and scaling dimensions of the Coulomb branch operator $Z$. These values can be found in [63] and are summarized in table 2 for later convenience. ${ }^{4}$

From there the anomalies and scaling dimensions in the IR can directly be computed from the previously derived equations. The only necessary information is the embedding index of the $\mathfrak{s u}(2)_{D}$ subalgebra defined by the nilpotent orbit. Since we only have one flavor symmetry factor, the Cartan matrix is uniquely specified by the nilpotent orbit one wants to consider. Then it is only a matter of evaluating the formulae of sections 3 and 4 .

\subsection{Plain mass deformations}

It is noteworthy that for all of the rank one probe D3-brane theories, the mesons never appear to decouple. However, $\Delta_{\mathrm{IR}}(Z)$ sometimes does decouple when the value of $r$ becomes too large. In general the unitarity bound for the operator $Z$ is violated whenever:

$$
\begin{array}{ll}
r \geq 5 & \text { for } \mathrm{SO}(8) \\
r \geq 19 & \text { for } E_{6} \\
r \geq 40 & \text { for } E_{7} \\
r \geq 107 & \text { for } E_{8} .
\end{array}
$$

There are a large number of possible nilpotent deformations. Due to the size of the resulting tables we only list our results for flavor symmetry $D_{4}$ and all rational results for the exceptional groups. Rational coefficients are of particular interest as they suggest additional structure present in the IR. When comparing our results with the subset of cases studied in [9] we find perfect agreement aside from the last column of table 5 which contains the correct value of $t_{*}$ but a minor typo for the values of $a_{\mathrm{IR}}$ and $c_{\mathrm{IR}}$.

The complete list of all the possible deformations can be accessed via a Mathematica routine summarized in appendix C. Due to the very large amount of data we only list here the rational results for the exceptional groups in appendix C.

The tables are organized as follows. For the top tables, first we list the Bala-Carter label of the deformation, or simply the partition of the fundamental representation's splitting

\footnotetext{
${ }^{4}$ While it is entirely possible to study nilpotent deformations of the Argyres-Douglas theories they are too simple to be of interest. However, for convenience we do list their UV values in table 2.
} 


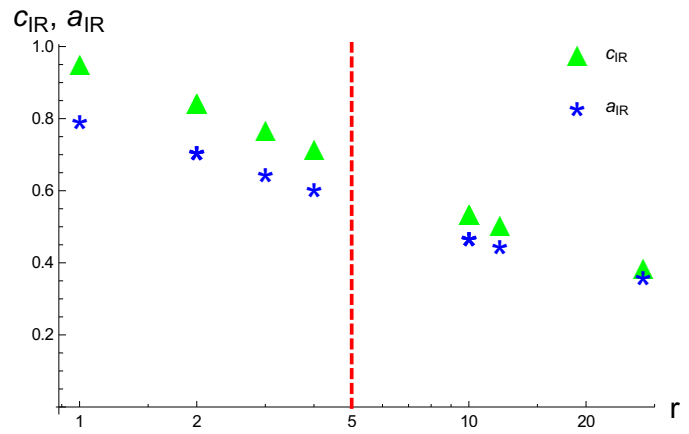

(a) $D_{4}$

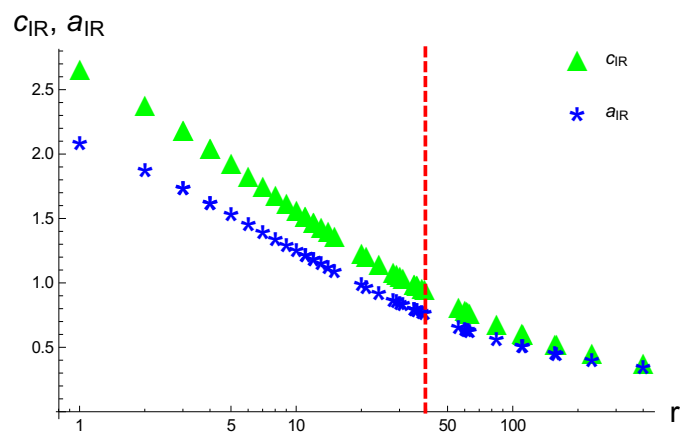

(c) $E_{7}$

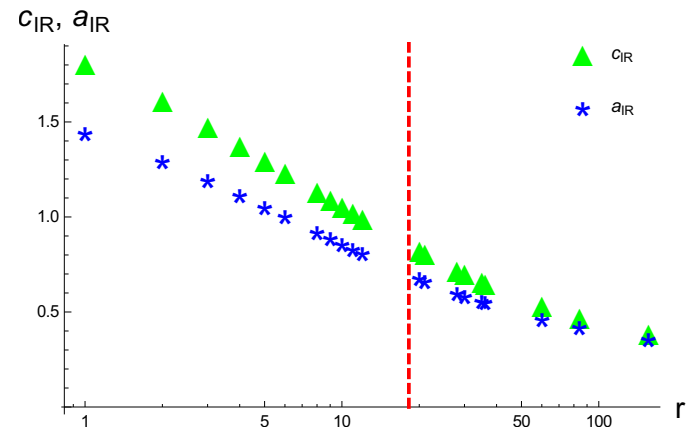

(b) $E_{6}$

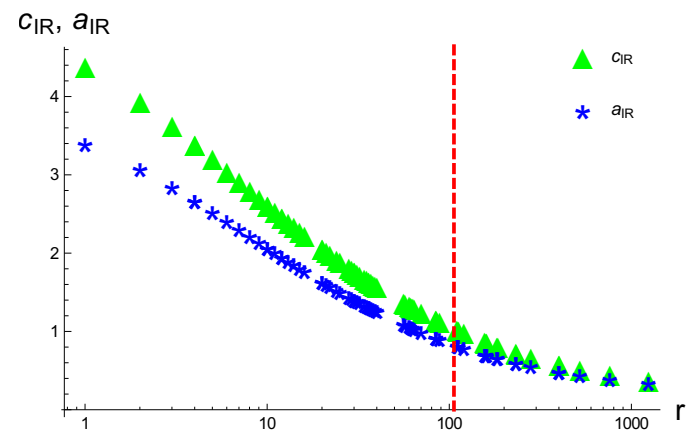

(d) $E_{8}$

Figure 4. Plots of $a_{\mathrm{IR}}$ (blue stars) and $c_{\mathrm{IR}}$ (green triangles) vs embedding index $r$ for the different probe D3-brane theories. The red vertical dashed line denotes the largest value of $r$ before the Coulomb branch operator $Z$ decouples. Anything to the right of this line has a single emergent $\mathrm{U}(1)$ to rescue the Coulomb branch operator. The plots are log-scaled on the x-axis for presentation purposes due to the fact that the region of deformed theories is denser around lower values of $r$ and becomes more sparse as $r$ increases.

in the case of $\mathrm{SO}(8)$. The second column gives the value of the embedding index $r$. The following three columns give the anomalies $a_{\mathrm{IR}}$ and $c_{\mathrm{IR}}$, as well as the value of the parameter $t$ after re-doing any $a$-maximization if necessary. Whenever fields decouple (because they first hit the unitarity bound and are rescued by emergent U(1)'s) then we can look at the interacting part versus the complete contribution to $a_{\mathrm{IR}}$ and $c_{\mathrm{IR}}$. Indeed, whenever an operator decouples it contributes a factor of $1 / 48$ or $1 / 24$ to $a_{\mathrm{IR}}$ and $c_{\mathrm{IR}}$ respectively, and we separately report these values in our tables. The first number in columns 3 and 4 is only the interacting piece, while the second number also includes the contribution from any free multiplets that decoupled. Thus those numbers only differ by an integer $n$ times $1 / 48$ (or $1 / 24$ ), where $n$ is equal to the number of multiplets generators that have decoupled and become free. If there is no emergent $\mathrm{U}(1)$ introduced and no field decouples then there is only an interacting piece and only the first number makes sense and is listed. Finally, the last two columns give the scaling dimension of the Coulomb branch parameter $Z$ and the lowest scaling dimension of the mesons $\mathcal{O}$ 's.

For the bottom tables we first list the Bala-Carter label of the deformation, followed by the residual flavor symmetry. The following four columns correspond to the flavor central 


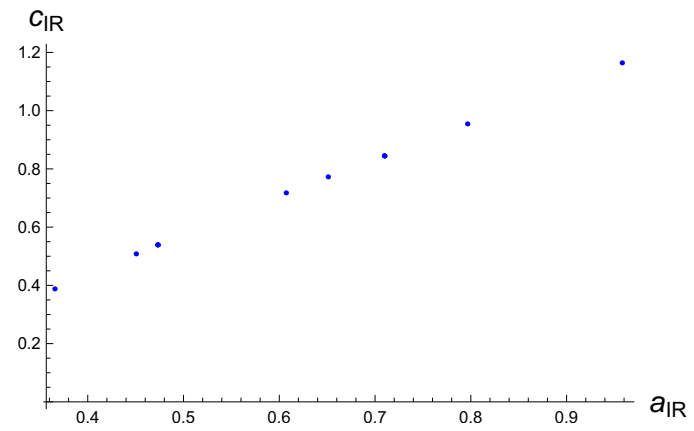

(a) $D_{4}$

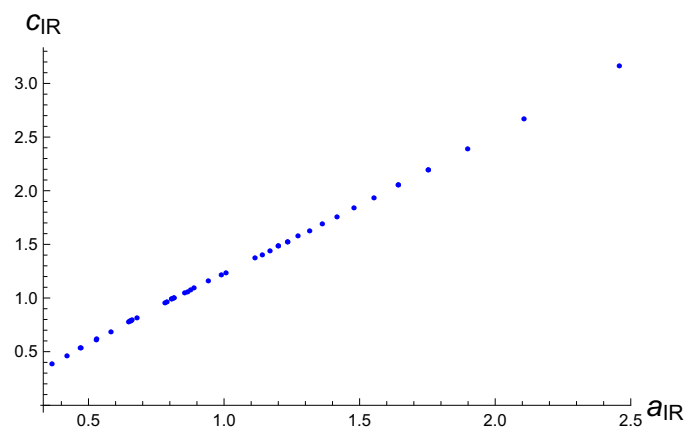

(c) $E_{7}$

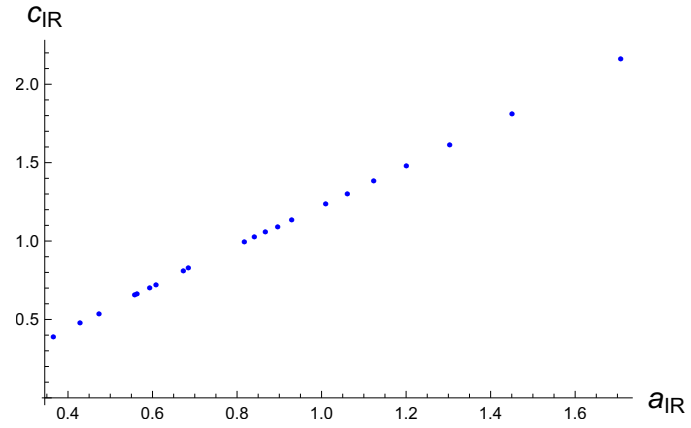

(b) $E_{6}$

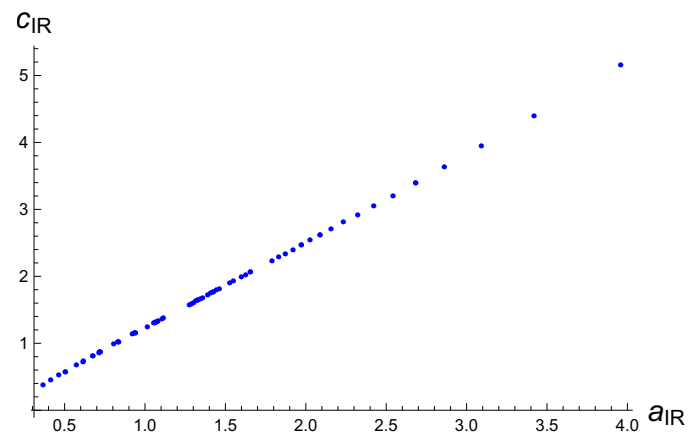

(d) $E_{8}$

Figure 5. Plots of $c_{\mathrm{IR}}$ vs. $a_{\mathrm{IR}}$ for plain nilpotent mass deformations of the different probe D3-brane theories.

charges $k_{\mathrm{IR}}$ taken with respect to the residual flavor symmetry. For each we list their value with only the interacting part of the theory or including the free fields which decoupled in seperate columns. Finally, we note that there are separate values for each of the subgroups in the product decomposition of the residual flavor, hence the multiple values listed in each column. For the theories with exceptional flavor symmetry we only list values that have rational anomalies.

Furthermore, as it is impractical to list all the other values in a single table we provide plots of $a_{\mathrm{IR}}$ and $c_{\mathrm{IR}}$ as functions of the embedding index $r$ :

As we can see, as $r$ increases, the anomalies decrease. Whenever an additional deformation is introduced the embedding index increases. Physically, this translates in a flow to a lower IR theory down the Hasse diagram of possible RG flows. As a result we expect the degrees of freedom to decrease, that is $a_{\mathrm{IR}}$ should decrease along this Hasse diagram. The fact that $a_{\mathrm{IR}}$ is a monotonically decreasing function of $r$ is an easy consistency check. We also note that the interacting piece of the anomaly (first value of columns 3 ) also decreases the same way.

It is also interesting to note that for a given $\mathrm{UV} \mathcal{N}=2$ fixed point, the ratio of anomalies $a_{\mathrm{IR}} / c_{\mathrm{IR}}$ remains roughly constant over the entire nilpotent network. Reference [49] noticed a similar effect. We also determine the overall statistical spread in the value of the ratio $a_{\mathrm{IR}} / c_{\mathrm{IR}}$ for plain mass deformations of the probe D3-brane theories. By inspection of 


\begin{tabular}{|c|c|c|c|c|}
\hline & $D_{4}$ & $E_{6}$ & $E_{7}$ & $E_{8}$ \\
\hline Mean & 0.86 & 0.83 & 0.82 & 0.81 \\
\hline Std. Dev. & 0.03 & 0.03 & 0.03 & 0.03 \\
\hline Max & 0.94 & 0.94 & 0.94 & 0.94 \\
\hline Min & 0.82 & 0.79 & 0.78 & 0.77 \\
\hline
\end{tabular}

Table 3. Table of means and standard deviations for the ratio $a_{\mathrm{IR}} / c_{\mathrm{IR}}$ across the entire nilpotent network defined by plain mass deformations of probe D3-brane theories. We also display the maximum and minimum values.

the plots in figure 4, we see that there is a roughly constant value for each theory. We also calculate the mean and standard deviation by sweeping over all such theories, the results of which are shown in table 3. Quite remarkably, the standard deviation is on the order of $1 \%$ to $5 \%$, indicating a remarkably stable value across the entire network of flows. Another curious feature is that the mean value of $a_{\mathrm{IR}} / c_{\mathrm{IR}}$ decreases as we increase to larger flavor symmetries. Precisely the opposite behavior is observed in the nilpotent networks of $4 \mathrm{D}$ conformal matter.

\subsection{Flipper field deformations}

Consider next flipper field deformations of the probe D3-brane theories. As one would expect, we recover the results from [18]. In appendix $\mathrm{C}$ we present all our results for $D_{4}$ flavor symmetry and only list the values with rational anomalies for the exceptional flavors $E_{6,7,8}$. Furthermore, we highlight cases where we obtain known enhancements to $\mathcal{N}=2$ theories such as $H_{0}, H_{1}$, and $H_{2}$ (as already pointed out in [18]), and we find an enhancement of the $E_{7}$ Minahan-Nemeschansky theory to the Argyres-Douglas theory $H_{1}$, in agreement with $[56,57,62]$. It is associated with the Bala-Carter label $E_{6}$ which has embedding index $r=156$. In such cases we can compute the embedding index $r_{F}$ of the residual flavor symmetry and see that not only $a_{\mathrm{IR}}$ and $c_{\mathrm{IR}}$ match the known values but $k_{\mathrm{IR}} r_{F}$ also yields the proper value for the flavor central charge of these theories. It is noteworthy that in those particular cases, the chiral multiplets, $M_{j_{(a)},-j_{(a)}}$, that survive transform trivially under the residual flavor symmetry and therefore do not introduce any additional contributions to the flavor central charge. This is however not true in general.

We also again plot $a_{\mathrm{IR}}$ and $c_{\mathrm{IR}}$ as functions of the embedding index $r$ for each of the above cases.

This time we see that the central charges do not exactly decrease as the embedding index $r$ increases. However, they do decrease along the flows defined by the Hasse-diagrams, as expected. Another interesting feature of these Hasse diagram flows is that the number of flipper field deformations which actually participate in a flow can vary wildly from orbit to orbit (since the number of $\mathfrak{s u}(2)_{D}$ irreducible representations also jumps a fair amount). Of course, such fields must be included in computing various anomalies, even if they serve to decouple mesonic operators which drop below the unitarity bound. Doing so, we find that $a_{\mathrm{IR}}$ indeed decreases monotonically along a flow. 


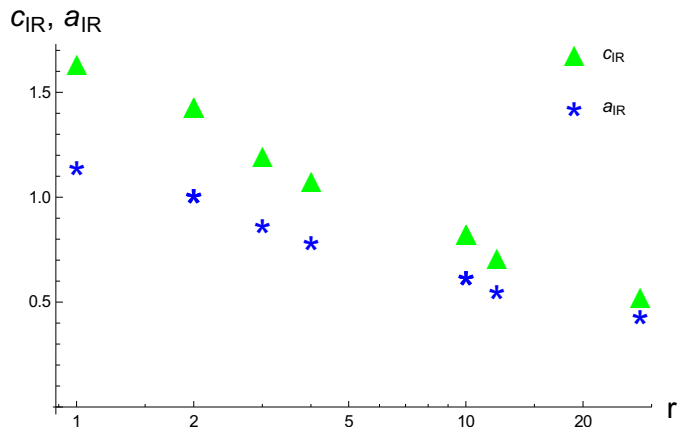

(a) $D_{4}$

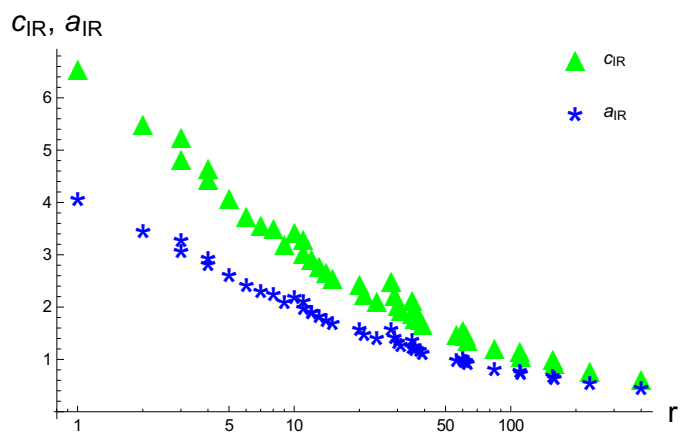

(c) $E_{7}$

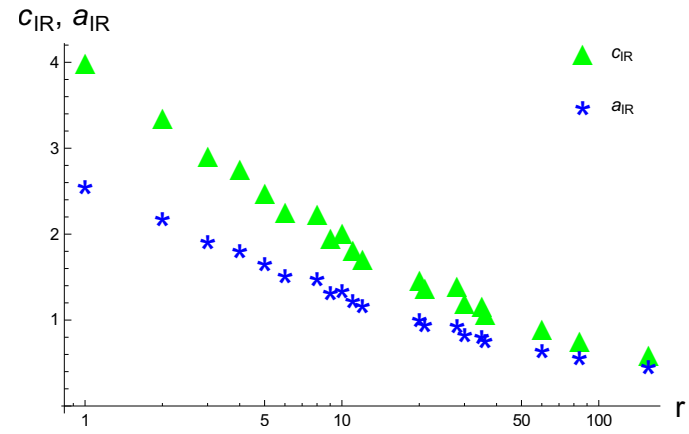

(b) $E_{6}$

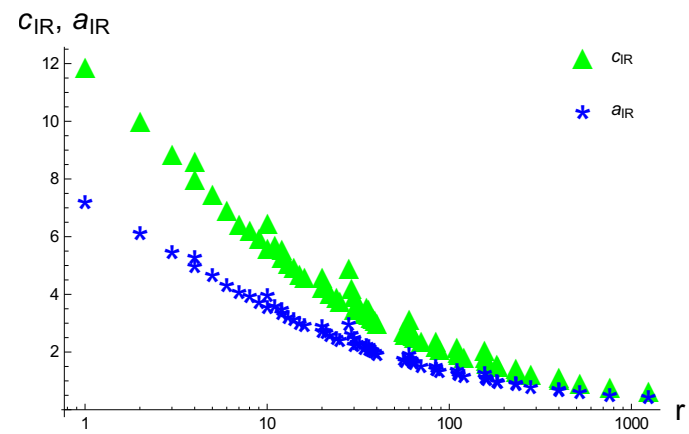

(d) $E_{8}$

Figure 6. Plots of $a_{\mathrm{IR}}$ (blue stars) and $c_{\mathrm{IR}}$ (green triangles) vs embedding index $r$ for the different flipper field deformations of probe D3-brane theories.

This raises the question of alternative numerical invariants instead of the embedding index which might be used to order RG flows in this class of theories. We have chosen the embedding index because this is the quantity which naturally appears in the construction of the infrared R-symmetry (see equations (3.14)). Additionally, it is numerically simple to obtain and often a useful proxy for the ordering of the RG flows. We are not aware of any other quantity which could provide a better trade off between accuracy and the complexity to compute it. Looking at the Hasse-diagram of the corresponding nilpotent orbits, one would expect that a more accurate description requires more parameters than just one. This would turn the presented plots into higher dimensional ones. For instance, the $x$-axis would need to be replaced by a series of branches corresponding to the full Hasse diagrams. The resulting plots would be much more complex than they need to be. Especially given how closely the embedding index gets to properly ordering the RG flows. Hence, we continue to rely on this physical parameter rather than try and introduce a less natural quantity.

Finally, another interesting feature of our analysis is that the ratio $a_{\mathrm{IR}} / c_{\mathrm{IR}}$ is roughly constant for a fixed deformation, given a flavor symmetry $\mathfrak{g}_{U V}$ in the UV (see figure 7). Much as for the plain nilpotent mass deformations, the overall statistical spread in the value of the ratio $a_{\mathrm{IR}} / c_{\mathrm{IR}}$ is also remarkably small, and is on the order of $1 \%$ to $5 \%$, indicating a remarkably stable value across the entire network of flows. Another curious feature is that 


\begin{tabular}{|c|c|c|c|c|}
\hline & $D_{4}$ & $E_{6}$ & $E_{7}$ & $E_{8}$ \\
\hline Mean & 0.73 & 0.69 & 0.67 & 0.65 \\
\hline Std. Dev. & 0.04 & 0.04 & 0.03 & 0.03 \\
\hline Max & 0.83 & 0.78 & 0.77 & 0.75 \\
\hline Min & 0.66 & 0.62 & 0.6 & 0.59 \\
\hline
\end{tabular}

Table 4. Table of means and standard deviations for the ratio $a_{\mathrm{IR}} / c_{\mathrm{IR}}$ across the entire nilpotent network defined by flipper field deformations of probe D3-brane theories. We also display the maximum and minimum values.

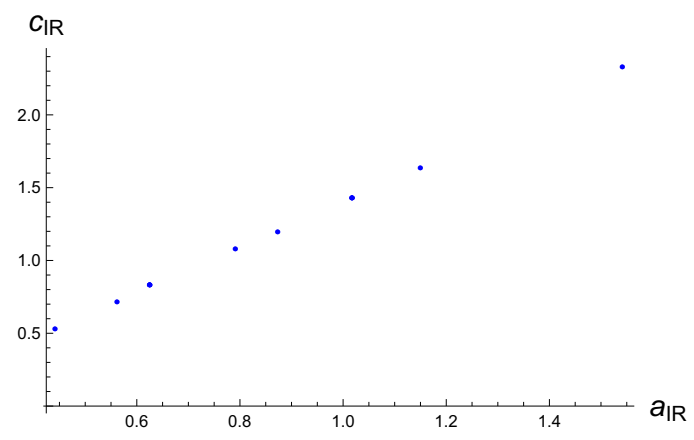

(a) $D_{4}$

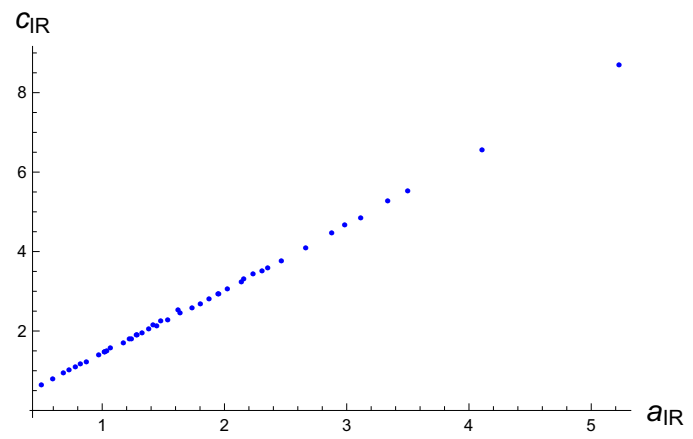

(c) $E_{7}$

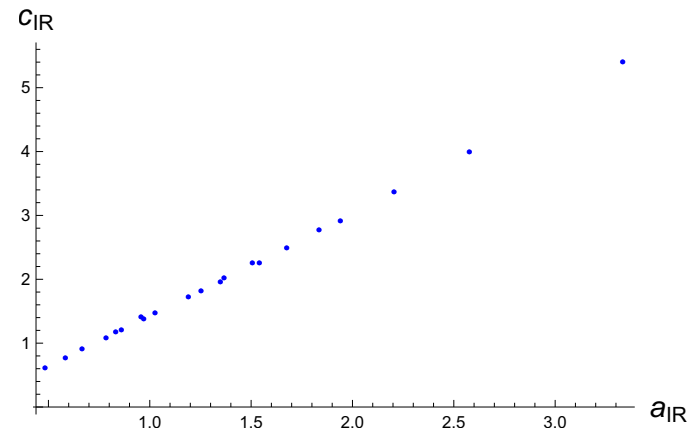

(b) $E_{6}$

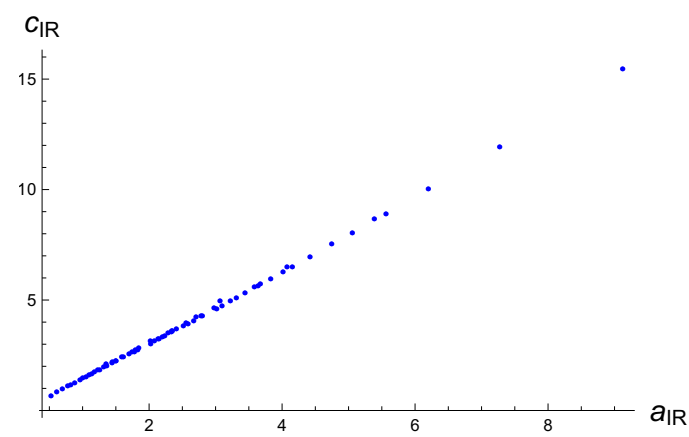

(d) $E_{8}$

Figure 7. Plots of $c_{\mathrm{IR}}$ vs. $a_{\mathrm{IR}}$ for the different flipper field deformations of probe D3-brane theories.

the mean value of $a_{\mathrm{IR}} / c_{\mathrm{IR}}$ decreases as we increase to larger flavor symmetries. Precisely the opposite behavior is observed in the nilpotent networks of $4 \mathrm{D}$ conformal matter. See table 4 for the specific values.

\section{$64 \mathrm{D}$ conformal matter theories}

In this section we turn to the case of $4 \mathrm{D}$ conformal matter theories. In F-theory terms, these are obtained from a pair of intersecting 7-branes each with gauge group $G$ which intersect along a common $T^{2}$, namely we have the compactification of $6 \mathrm{D}$ conformal matter to an $\mathcal{N}=2$ theory. Some properties of these theories such as the anomaly polynomial were determined in $[46,47]$, and their role as building blocks in generalized quiver gauge theories was studied in [64, 65]. 


\begin{tabular}{|c|c|c|c|c|}
\hline$\left(G_{L}, G_{R}\right)$ & $\left(D_{k}, D_{k}\right)$ & $\left(E_{6}, E_{6}\right)$ & $\left(E_{7}, E_{7}\right)$ & $\left(E_{8}, E_{8}\right)$ \\
\hline$a_{\mathrm{UV}}$ & $\frac{1}{24}(k(14 k-19)-53)$ & $\frac{613}{24}$ & $\frac{817}{12}$ & $\frac{1745}{8}$ \\
\hline$c_{\mathrm{UV}}$ & $\frac{1}{6}(k(4 k-5)-13)$ & $\frac{173}{6}$ & $\frac{442}{6}$ & $\frac{457}{2}$ \\
\hline$k_{L}^{\text {flav }}, k_{R}^{\text {flav }}$ & $4 k-4$ & 24 & 36 & 60 \\
\hline \multirow{2}{*}{$\Delta\left(Z_{i}\right)$} & \multirow{2}{*}{$6_{1}, \ldots,(2 k-2)_{1}$} & $\left\{6_{1}, 8_{1}, 9_{1}\right.$, & $\left\{6_{1}, 8_{1}, 10_{1}\right.$, & $\left\{6_{1}, 8_{1}, 12_{2}, 14_{2}\right.$, \\
& & $\left.12_{2}\right\}$ & $\left.12_{2}, 14_{2}, 18_{3}\right\}$ & $\left.18_{3}, 20_{3}, 24_{4}, 30_{5}\right\}$ \\
\hline
\end{tabular}

Table 5. Anomalies and scaling dimensions for $4 \mathrm{D} \mathcal{N}=2(G, G)$ conformal matter. In the last row, the subscripts are the multiplicities, i.e. the number of Coulomb branch operators with that specific scaling dimension.

Now, in this case, the interpretation of the mass parameters is somewhat different from the D3-brane case. The reason is that the $4 \mathrm{D}$ conformal matter defines a current which couples to the gauge fields of the 7 -brane. More precisely, from the $(0,1)$ connection and the adjoint valued $(2,0)$-form, it is now the pullback of the $(0,1)$ connection $\mathbb{A}_{(0,1)}$ onto the $T^{2}$ which actually couples to the $4 \mathrm{D}$ conformal matter. A mass deformation then corresponds to switching on a zero mode for this connection along the curve. Now in the case where the associated Wilson loop is not unipotent (so that the zero mode is not nilpotent), this would be an element of the Deligne cohomology $\mathcal{D}_{2,2}\left(C Y_{4}\right)$ for the associated elliptically fibered Calabi-Yau fourfold of the F-theory model (see [31] as well as [33]). This can also be viewed as a T-brane deformation of sorts, because in the limit where the mass parameter is nilpotent, this deformation is "invisible" in the associated moduli space problem. ${ }^{5}$ Clearly, it is also natural to promote these background parameters to a dynamical field, as will happen if we wrap these 7-branes on compact Kähler surfaces, and some examples of weakly gauging flavor symmetries in this way were studied in [65]. To get a stringy embedding of the flipper fields, however, we must take a suitable limit where the gauge fields become IR free, but the chiral superfields remain dynamical.

Our plan in the remainder of this section will be to discuss some further aspects of these conformal matter theories. We begin by reviewing some aspects of the original $\mathcal{N}=2$ theories, and then turn to an analysis of the resulting nilpotent network of $\mathcal{N}=1$ fixed points. When we turn to the plots and statistics for these networks, we treat the nilpotent orbit with $G_{L} \leftrightarrow G_{R}$ interchanged as distinct.

\subsection{Summary of UV $\mathcal{N}=2$ fixed points}

We now review some aspects of $\mathcal{N}=2(G, G) 4 \mathrm{D}$ conformal matter obtained from compactification of $(G, G) 6 \mathrm{D}$ conformal matter on a $T^{2}$. We present in table 5 the values for the central charges and flavor symmetries, together with the dimensions and multiplicities of the Coulomb branch operators. We give further details on how those results are obtained in appendix B.

\footnotetext{
${ }^{5}$ More precisely, the moduli space can develop singularities, and as explained in [33], the gauge theory on the 7-brane serves to complete the moduli space in these singular limits.
} 
The dimension of the Coulomb branch for the different conformal matter theories on $T^{2}$ are

$$
\begin{aligned}
\operatorname{dim}_{\mathbb{C}}\left(\operatorname{Coul}\left[\left(D_{k}, D_{k}\right)\right]\right) & =k-3, \\
\operatorname{dim}_{\mathbb{C}}\left(\operatorname{Coul}\left[\left(E_{6}, E_{6}\right)\right]\right) & =5, \\
\operatorname{dim}_{\mathbb{C}}\left(\operatorname{Coul}\left[\left(E_{7}, E_{7}\right)\right]\right) & =10, \\
\operatorname{dim}_{\mathbb{C}}\left(\operatorname{Coul}\left[\left(E_{8}, E_{8}\right)\right]\right) & =21,
\end{aligned}
$$

which matches the expectation from 6D [44]:

$$
\left.\operatorname{dim}_{\mathbb{C}}(\operatorname{Coul}[G])\right)=h_{G}^{\vee}-r_{G}-1,
$$

where $r_{G}$ is the rank of $G$ and $h_{G}^{\vee}$ is the dual Coxeter number of $G$. In order to extract the dimensions of the Coulomb branch operators for the different conformal matter theories, we read off the scaling dimension of the deformations from the mirror geometries of the elliptic threefold of the F-theory geometry. The mirror geometries for $\left(E_{n}, E_{n}\right)$ theories were provided in [48] and the $\left(D_{k}, D_{k}\right)$ case can be obtained from the curve in equation (5.4) of reference [46].

\subsection{Plain mass deformations}

The computations for conformal matter follow the general procedure outlined in previous sections. We now have two flavor groups, so two nilpotent orbits labelled by corresponding Bala-Carter labels. Each one comes with an embedding index $r_{L}$ and $r_{R}$.

We have actually already encountered the $\left(D_{4}, D_{4}\right) 4 \mathrm{D}$ conformal matter theory: it is simply the rank one $E_{8}$ Minahan-Nemeschansky theory (it can still be accessed with the code described in appendix C). It mainly serves as a cross-check on the general procedure, and we find perfect agreement for those deformations which live in an $\mathfrak{s o}(8) \times \mathfrak{s o}(8)$ subalgebra. Thus, we simply list in appendix $\mathrm{C}$ the rational theories in the case where the parent $4 \mathrm{D}$ conformal matter theory has exceptional flavor symmetry. Due to their large size the tables are also split in their length. The top half contains the Bala-Carter labels, embedding indices, anomalies and $t_{*}$. The bottom half repeats the Bala-Carter labels and $t_{*}$ before providing scaling dimensions. Finally, the tables for the flavor central charges are too large to include here. So, we refer the reader to the companion Mathematica code for those results.

We also provide contour plots of $a_{\mathrm{IR}}$ vs. the embedding indices of the right and left flavors. We hasten to add that while the partial ordering of nilpotent orbits enforces a corresponding ordering for the associated embedding indices, the converse is not true (the Hasse diagram has more fine structure). This is an unfortunate artifact of displaying all of our data with respect to a two-dimensional contour plot. Of course, the plots (just like the tables) are symmetric under the interchange of $r_{L}$ with $r_{R}$. We also see that for any fixed value of $r_{L}$ the value of $a_{\mathrm{IR}}$ decreases as the deformation on the right increases (along the Hasse diagram) when the interacting piece plus free decoupled fields are considered, as well as when central charges of only the interacting piece are analyzed (the plots for only the interacting piece would look very similar). 


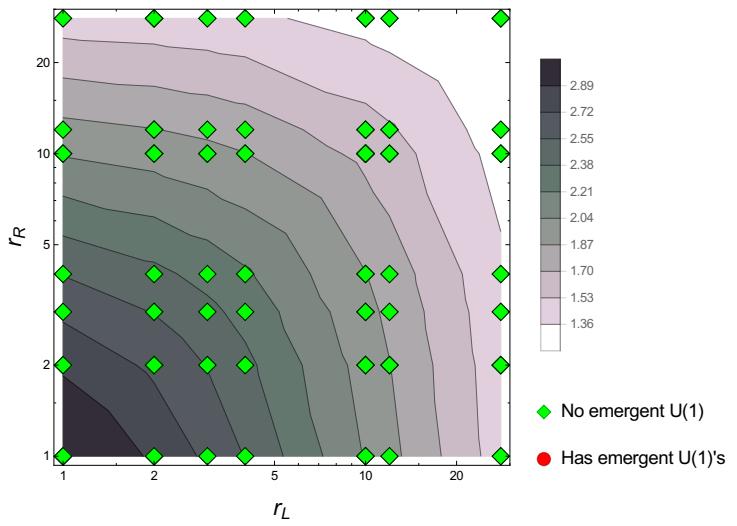

(a) $\left(D_{4}, D_{4}\right)$

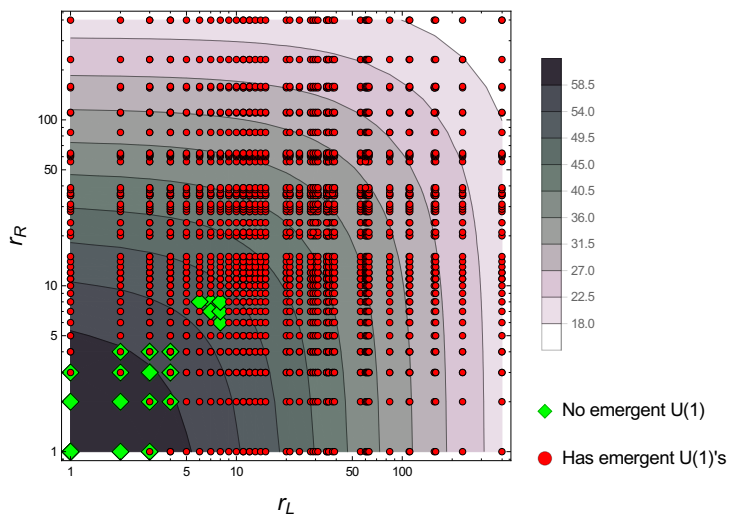

(c) $\left(E_{7}, E_{7}\right)$

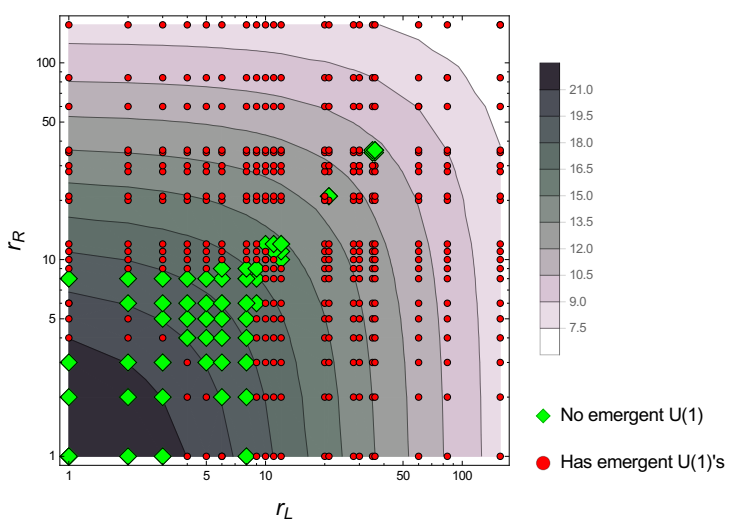

(b) $\left(E_{6}, E_{6}\right)$

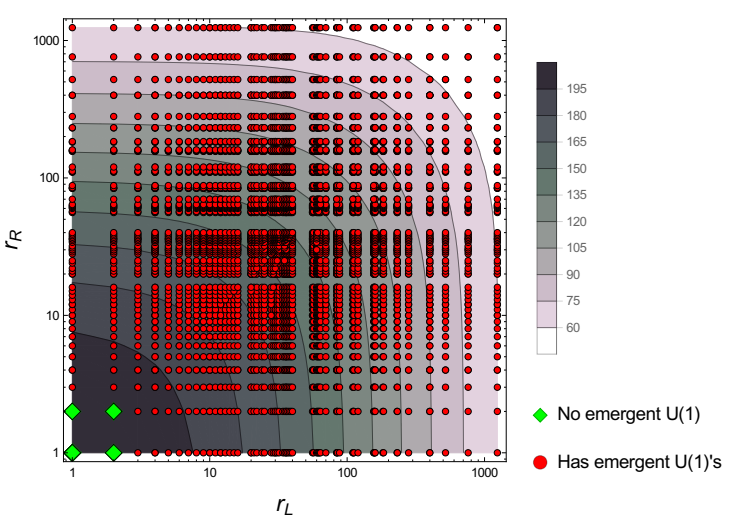

(d) $\left(E_{8}, E_{8}\right)$

Figure 8. Plots of $a_{\mathrm{IR}}$ vs left and right embedding indices for the different plain mass nilpotent deformations of $4 \mathrm{D}$ conformal matter theories. The contour plots are obtained by extrapolating between the actual data points which are labelled in green diamonds and red circles. The green diamonds correspond to deformations where all operators remain above the unitarity bound and no emergent $\mathrm{U}(1)$ appears. The red circles correspond to deformations where some operators hit the unitarity bound and emergent U(1)'s are present. We emphasize that sometimes different nilpotent orbits can have the same embedding index. A log-scale is used to spread the dense region at low values of the embedding indices.

Furthermore, if we simultaneously increase both $r_{L}$ and $r_{R}$ while keeping $r_{L}=r_{R}$ (along the Hasse diagram), then $a_{\mathrm{IR}}$ monotonically decreases. This is again consistent with the expectation that the number of degrees of freedom should decrease as the deformations becomes larger along the RG flows.

Another interesting feature of our numerical sweep is that we sometimes encounter theories where an operator decouples, but further down the Hasse diagram, we see no apparent unitarity bound violations. This does not contradict the general structure implied by the nilpotent cone, since deeper down in the Hasse diagram it often happens that the top spin operator of $\mathfrak{s u}(2)_{D}$ may not be a top-spin operator deeper down in the nilpotent cone. As we have already explained, the lower spin operators are trivial in the chiral ring of the IR fixed point, so it is neither here nor there to see a jump in the number of emergent $\mathrm{U}(1)$ 's as we proceed deeper into the IR. 


\begin{tabular}{|c|c|c|c|c|}
\hline & $\left(D_{4}, D_{4}\right)$ & $\left(E_{6}, E_{6}\right)$ & $\left(E_{7}, E_{7}\right)$ & $\left(E_{8}, E_{8}\right)$ \\
\hline Mean & 0.80 & 0.91 & 0.94 & 0.97 \\
\hline Std. Dev. & 0.01 & 0.01 & 0.004 & 0.003 \\
\hline Max & 0.81 & 0.91 & 0.95 & 0.98 \\
\hline Min & 0.77 & 0.89 & 0.92 & 0.96 \\
\hline
\end{tabular}

Table 6. Table of means and standard deviations for the ratio $a_{\mathrm{IR}} / c_{\mathrm{IR}}$ across the entire nilpotent network defined by plain mass deformations of $4 \mathrm{D}$ conformal matter. We also display the maximum and minimum values.

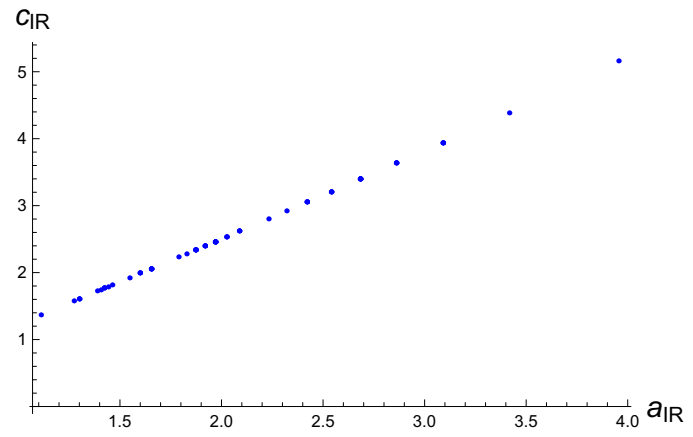

(a) $\left(D_{4}, D_{4}\right)$

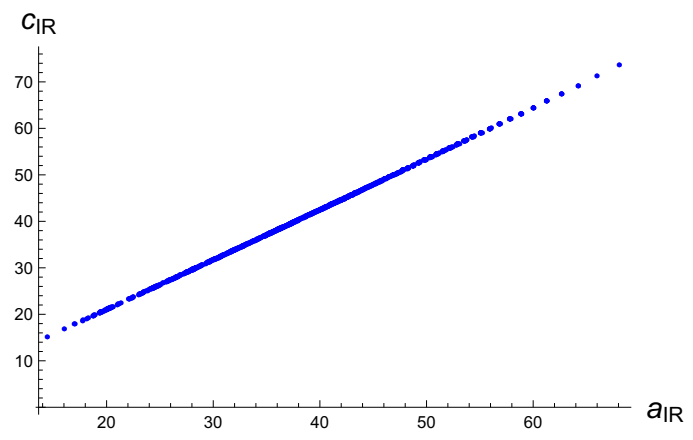

(c) $\left(E_{7}, E_{7}\right)$

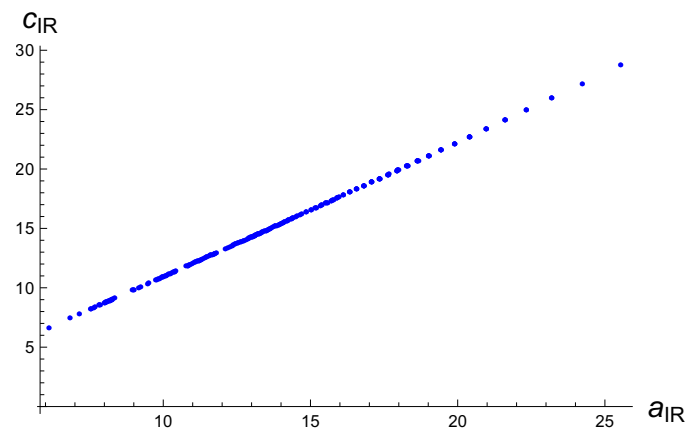

(b) $\left(E_{6}, E_{6}\right)$

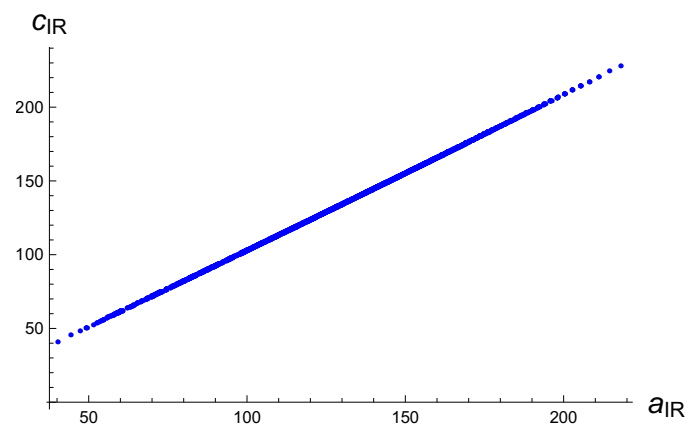

(d) $\left(E_{8}, E_{8}\right)$

Figure 9. Plots of $c_{\mathrm{IR}}$ vs $a_{\mathrm{IR}}$ for the different plain mass nilpotent deformations of $4 \mathrm{D}$ conformal matter theories.

We also determine the overall statistical spread in the value of the ratio $a_{\mathrm{IR}} / c_{\mathrm{IR}}$ for plain mass deformations of the probe D3-brane theories. By inspection of the plots in figure 9 , we see that there is a roughly constant value for each theory. We also calculate the mean and standard deviation by sweeping over all such theories. Just as in the case of the probe D3-brane theories, we find that the standard deviation is on the order of $1 \%$ to $5 \%$, indicating a remarkably stable value across the entire network of flows. The specific values are displayed in table 6 . Another curious feature is that the mean value of $a_{\mathrm{IR}} / c_{\mathrm{IR}}$ increases as we go to larger UV flavor symmetries. Precisely the opposite behavior is observed in the nilpotent networks of probe D3-brane theories. 


\begin{tabular}{|c|c|c|c|c|}
\hline & $\left(D_{4}, D_{4}\right)$ & $\left(E_{6}, E_{6}\right)$ & $\left(E_{7}, E_{7}\right)$ & $\left(E_{8}, E_{8}\right)$ \\
\hline Mean & 0.73 & 0.87 & 0.92 & 0.96 \\
\hline Std. Dev. & 0.01 & 0.02 & 0.01 & 0.01 \\
\hline Max & 0.80 & 0.91 & 0.95 & 0.98 \\
\hline Min & 0.68 & 0.81 & 0.86 & 0.91 \\
\hline
\end{tabular}

Table 7. Table of means and standard deviations for the ratio $a_{\mathrm{IR}} / c_{\mathrm{IR}}$ across the entire nilpotent network defined by flipper field deformations of $4 \mathrm{D}$ conformal matter. We also display the maximum and minimum values.

\subsection{Flipper field deformations}

Finally, we come to flipper field deformations of conformal matter. The analysis is simplified by the fact that we do not need to worry about mesons decoupling since they are automatically rescued (if they drop below the unitarity bound) by the flipper fields $M$ to which they couple.

As before, the results with rational values are tabulated in appendix $\mathrm{C}$, and more general deformations can be accessed via the Mathematica code.

Finally, we provide contour plots of $a_{\mathrm{IR}}$ vs. the left and right embedding indices $r_{L}$ and $r_{R}$. Again, we emphasize that what really needs to be monotonic is the flow down the Hasse diagram, which in most cases (though not all) aligns with the increase of the embedding indices $r_{L}$ and $r_{R}$. Quite remarkably, even this coarse data based on the embedding indices (though there are a few exceptions) usually is enough to establish monotonicity.

We also determine the overall statistical spread in the value of the ratio $a_{\mathrm{IR}} / c_{\mathrm{IR}}$ for flipper field deformations of $4 \mathrm{D}$ conformal matter. By inspection of the plots in figure 11, we see that there is a roughly constant value for each theory. We also calculate the mean and standard deviation by sweeping over all such theories, displaying the results in table 7 . As in all the other cases we have considered, the standard deviation is on the order of $1 \%$ to $5 \%$, indicating a remarkably stable value across the entire network of flows. Another curious feature is that the mean value of $a_{\mathrm{IR}} / c_{\mathrm{IR}}$ increases as we increase to larger flavor symmetries. Precisely the opposite behavior is observed in the nilpotent networks of probe D3-brane theories.

\section{Conclusions}

One of the important open issues in the study of conformal field theories is to better understand the totality of fixed points, and their network of flows under deformations. In this paper we have shown that a great deal of information on the structure of RG flows for 4D SCFTs can be extracted in the special case of nilpotent mass deformations. Starting from a UV $\mathcal{N}=2$ SCFT, we have presented a general analysis of the resulting $\mathcal{N}=1$ fixed points, both in the case of plain mass deformations, as well as in the generalization to flipper field deformations, where these parameters are treated as background vevs for a dynamical adjoint valued $\mathcal{N}=1$ chiral superfield of the parent theory. In addition to presenting a general analysis of the resulting fixed points, we have performed an explicit sweep over all 


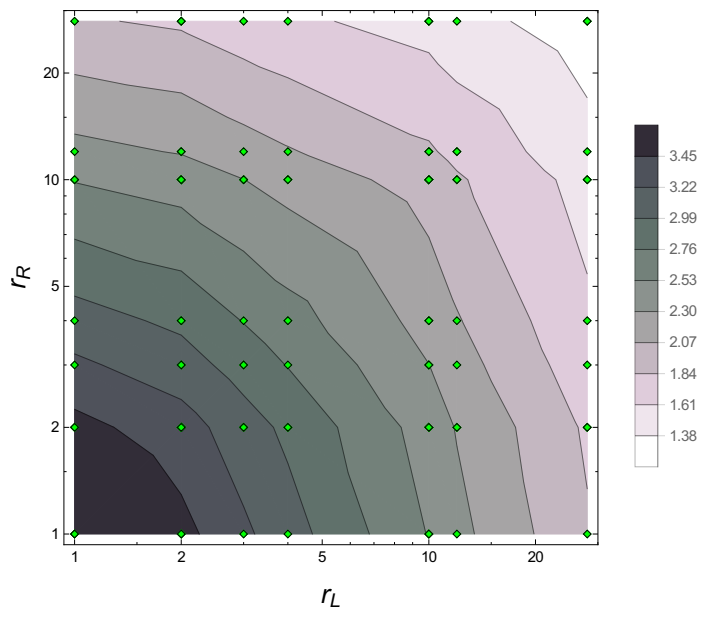

(a) $\left(D_{4}, D_{4}\right)$

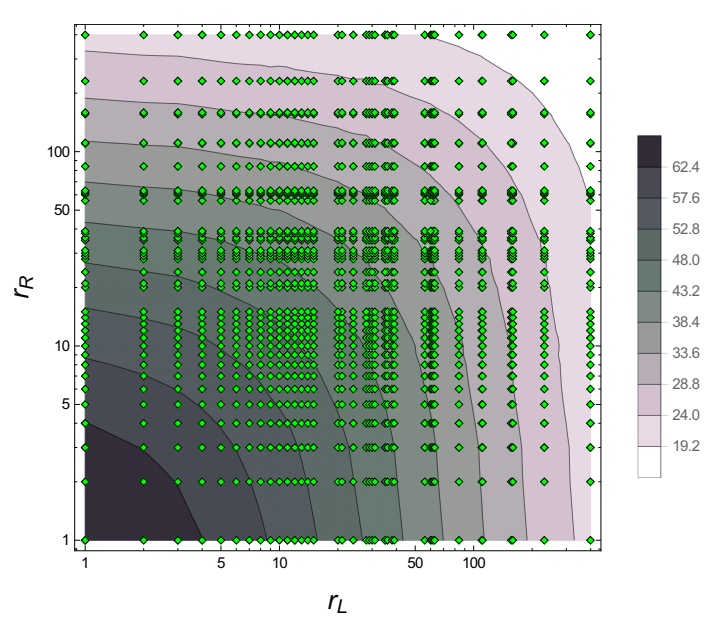

(c) $\left(E_{7}, E_{7}\right)$

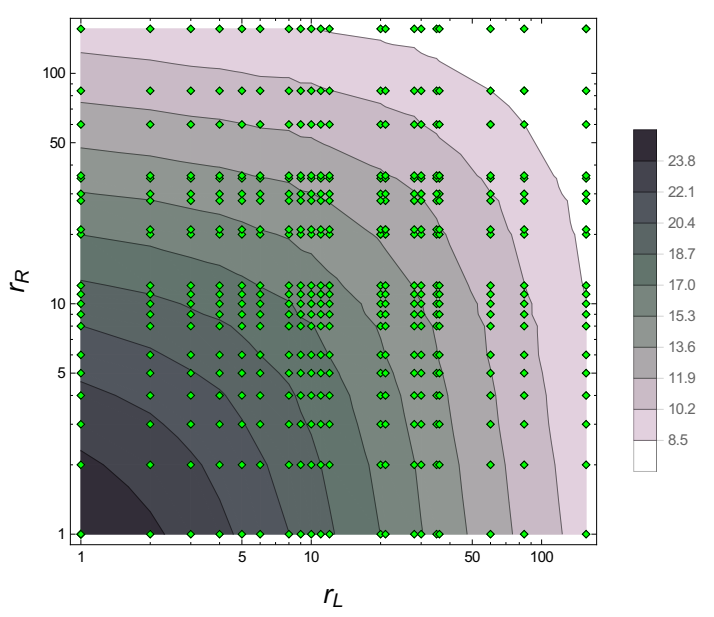

(b) $\left(E_{6}, E_{6}\right)$

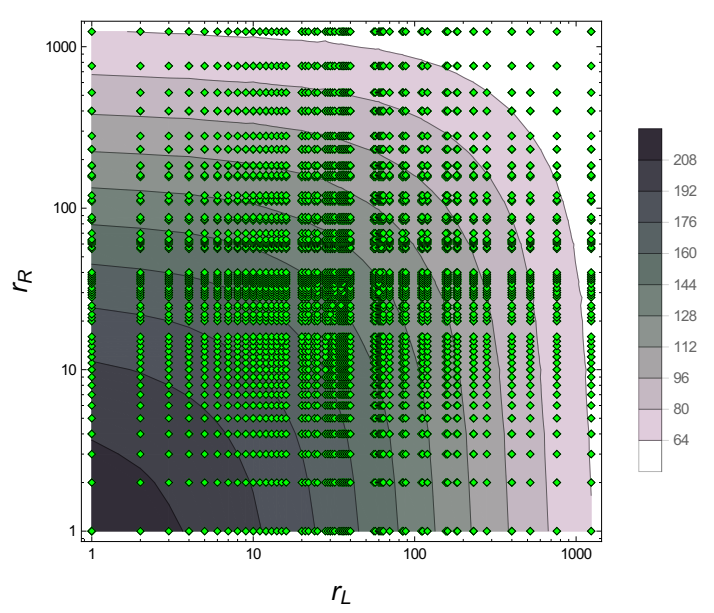

(d) $\left(E_{8}, E_{8}\right)$

Figure 10. Plots of $a_{\mathrm{IR}}$ vs left and right embedding indices for the different conformal matter theories, with flipper field deformations. The contour plots are obtain by extrapolating between the actual data points which are labelled in green.

possible nilpotent deformations for the $\mathcal{N}=2$ theories defined by D3-branes probing a $D$ or $E$-type 7-brane, as well as the nilpotent deformations of $4 \mathrm{D}(G, G)$ conformal matter. In both cases, we have found strong evidence that the mathematical partial ordering defined by the nilpotent cone of the associated Lie algebras is obeyed in the physical theories as well. Moreover, the directed graph of this partially ordered set also lines up with the possible relevant deformations of the physical theory, providing a very detailed picture of the possible RG flows from one fixed point to another. The structure of the Hasse diagrams obtained provides a partially ordered set, which cleanly matches to physical 4D RG flows. We can then take advantage of this fact (even in a more general setting) whenever there is a flavor symmetry present and we activate a breaking pattern generated by a nilpotent orbit. 


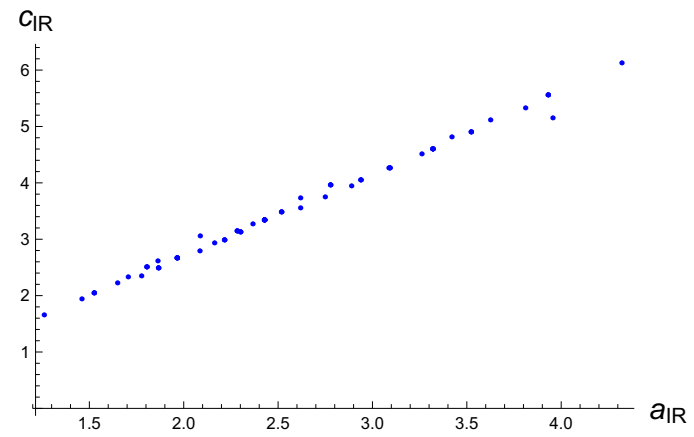

(a) $\left(D_{4}, D_{4}\right)$

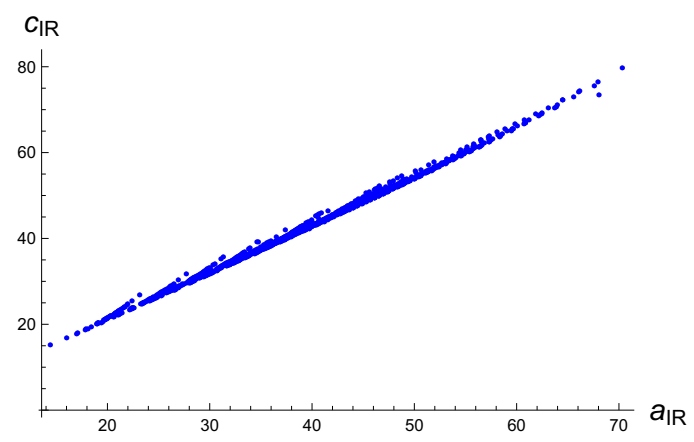

(c) $\left(E_{7}, E_{7}\right)$

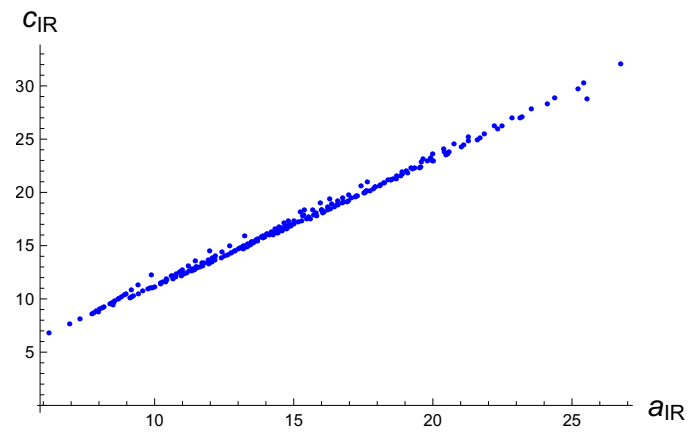

(b) $\left(E_{6}, E_{6}\right)$

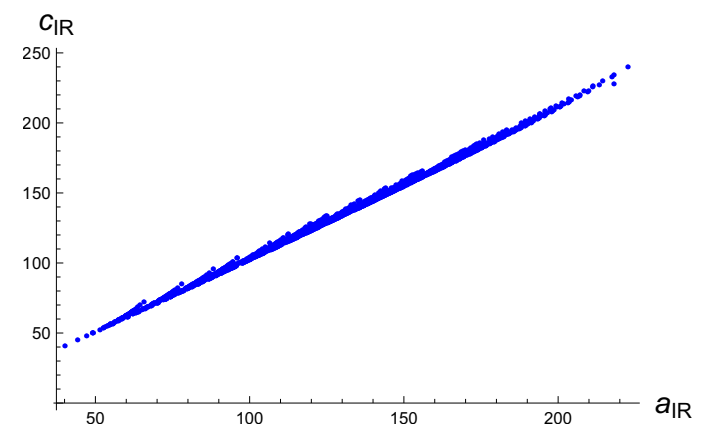

(d) $\left(E_{8}, E_{8}\right)$

Figure 11. Plots of $c_{\mathrm{IR}}$ vs. $a_{\mathrm{IR}}$ for flipper field deformations of $4 \mathrm{D}$ conformal matter.

In addition to presenting the full sweep over theories in a companion Mathematica program, we have also observed a number of intriguing "phenomenological" features, including the appearance of several theories with rational anomalies. We have also seen that for a given $\mathrm{UV} \mathcal{N}=2$ fixed point, the ratio $a_{\mathrm{IR}} / c_{\mathrm{IR}}$ is roughly constant over the entire nilpotent network. In the remainder of this section we discuss some avenues of further investigation.

One item left open by our analysis is a full treatment of the full network of RG flows in cases where mesonic operators decouple from the new IR fixed point. As we have already explained, such mesonic operators are often necessary to perform further perturbations deeper down in the Hasse diagram, so the absence of these operators could a priori pose some issues in the context of matching the full network defined by the Hasse diagram to corresponding RG flows. Even so, we have not found an explicit example which demonstrates that any links are in fact "broken." It would be most illuminating to further understand this class of theories.

Even within the class of theories considered here, there are some additional relevant deformations we could contemplate switching on. This includes the possibility of mass deformations which are semi-simple, namely their matrix representatives are diagonalizable. Since such diagonal elements can also be presented as the sum of two nilpotent elements, it is quite likely that the analysis presented here may implicitly cover such cases as well, and may actually help to "explain" the appearance of our rational theories. It would be interesting to analyze this issue further. 
The bulk of this paper has focussed on determining various properties of the new infrared fixed points generated by nilpotent mass deformations, including the operator scaling dimensions of various operators. Another tractable quantity to potentially extract is the superconformal index. This could shed additional light on the IR properties of these theories. Additionally, it would be quite interesting to see whether there is a corresponding partial ordering for these indices, as induced by the partial ordering on nilpotent orbits.

Much of our analysis has focussed on the case of a single D3-brane probing an F-theory 7-brane, as well as the case of "rank one 6D conformal matter," namely (in M-theory terms) a single M5-brane probing an ADE singularity. It would be quite natural to extend the analysis presented here to the case of additional branes. While the anomalies for the case of multiple D3-branes have already been determined [63], the corresponding statements for multiple M5-branes probing an ADE singularity, and the resulting 4D anomaly polynomial are apparently unknown. With this result in hand, it would then be possible to study nilpotent mass deformations for this class of theories as well.

Another natural class of theories involves the compactification of $6 \mathrm{D}$ conformal matter on more general Riemann surfaces in the presence of background fluxes and punctures. In this case, even before switching on nilpotent mass deformations, we expect from the general procedure outlined in [66] to get a $4 \mathrm{D} \mathcal{N}=1 \mathrm{SCFT}$, as in references [46-48, 64, 65, 6777]. Many of these theories admit a weakly coupled Lagrangian description [77, 78], so studying the possible nilpotent deformation and comparing the central charges with the class of theories studied here might lead to Lagrangian descriptions for some of the resulting IR fixed points.

Finally, we have also seen a number of numerical coincidences, including the appearance of rational theories, as well as a relatively constant value for $a_{\mathrm{IR}} / c_{\mathrm{IR}}$ over an entire nilpotent network. It would be very interesting to understand whether these coincidences have a simple top down interpretation.

\section{Acknowledgments}

We thank J. Kaidi, M. Fazzi, S. Giacomelli, T. Rudelius and F. Xu for useful discussions. FA, JJH and TBR also thank the 2018 Summer Workshop at the Simons Center for Geometry and Physics for hospitality during the completion of this work. The work of FA, FH, JJH, and TBR is supported by NSF CAREER grant PHY-1756996. The work of FA and FH is also supported by NSF grant PHY-1620311.

\section{A The embedding index}

The embedding index $r$ here refers to that of a splitting of the group $G=D_{4}$, or $E_{6,7,8}$ into irreducible representations (irreps) of SU(2). There are two equivalent ways of computing this embedding index $r$. The first method is by computing the sum of the indices of the $\mathrm{SU}(2)$ irreps divided by the index of the representation of the group $G$ being split. That is, given a representation $\rho(G)$ of $G$ and the branching $\rho(G) \rightarrow m_{1} \mathbf{n}_{1}+m_{2} \mathbf{n}_{2}+\ldots$ where 
$m_{(a)}$ are multiplicities and $\mathbf{n}_{(a)}$ are $\mathrm{SU}(2)$ irreps, the embedding index is given by:

$$
r=\frac{\sum_{(a)} m_{(a)} \cdot \operatorname{ind}\left(\mathbf{n}_{(a)}\right)}{\operatorname{ind}(\rho(G))} .
$$

For instance the splitting of $D_{4}$ according to the partition $[5,3]$ gives: $\mathbf{2 8} \rightarrow 3(\mathbf{3})+(\mathbf{5})+2(\mathbf{7})$ so that

$$
r=\frac{3 \times 4+20+2 \times 56}{12}=12
$$

As we can see, this definition of the embedding index is representation independent. However it requires that we know the branching rule of splitting of $G$ to $\mathrm{SU}(2)$ caused by the deformation of interest.

For this reason, we turn to the second method which makes use of the decorated Dynkin diagrams provided in [79] for the exceptional groups. Their labels specify a vector $v$ in the Cartan subalgebra which then yields the projection matrix $\mathbb{P}=v \cdot C_{\mathfrak{g}}^{-1} \cdot C_{\mathfrak{g}}$ is the Cartan matrix of the Lie algebra $\mathfrak{g}$, and $\mathbb{P}$ is the projection matrix of the weights of $\mathfrak{g}$ into the $\mathrm{SU}(2)_{D}$ nilpotent subalgebra. As a result the decorated Dynkin diagrams can be directly used to obtain the branching rules and the embedding indices,

$$
r=\frac{1}{2} \operatorname{Tr}\left(v \cdot C_{\mathfrak{g}}^{-1} \cdot v^{T}\right)
$$

where the $\frac{1}{2}$ coefficient is simply a normalization factor.

Now, for $D_{4}$ we do not have the decorated Dynkin diagrams readily available to us, so we need to compute them. We start with the 12 possible partitions of $\mathrm{SO}(8)$ provided by [80]. Following this procedure along with [81] one can obtain the vectors $v$ for $\mathrm{SO}(2 k)$ in the same form as the ones provided by [79] for the exceptional groups. In summary the procedure is as follows:

We begin by listing the possible partitions of $\mathrm{SO}(2 k): p_{i}=\left\{n_{l}\right\}$ where $i$ runs over the number of possible nilpotent deformations of $\mathrm{SO}(2 k)$ and $n_{l}$ are integers summing to $2 k$. The nilpotent deformation defines an $\mathrm{SU}(2)$ subalgebra $[H, X]=2 X,\left[H, X^{\dagger}\right]=-2 X^{\dagger}$, $\left[X, X^{\dagger}\right]=H$ where $X$ is the nilpotent orbit/deformation. $X$ is directly constructed from the partitions: $X$ is a $2 k \times 2 k$ matrix filled on the first superdiagonal by the Jordan blocks corresponding to the $\mathrm{SU}(2)$ irreps defined by the partitions. Namely $\sqrt{j(j+1)-m(m+1)}$ where $-j \leq m \leq j-1$. For instance, the $\mathrm{SO}(10)$ partition $\{7,3\}$ yields two Jordan blocks. $X$ is zero everywhere except on the first super diagonal which is given by the list $(\sqrt{6}, \sqrt{10}, \sqrt{12}, \sqrt{12}, \sqrt{10}, \sqrt{6}, 0, \sqrt{2}, \sqrt{2})$ where for the first block (which defines the first 6 entries) we have $j=3$ and for the second block (which defines the last 2 entries) we have $j=1$.

Then the corresponding Cartan matrix $H$ is given by $\left[X, X^{\dagger}\right]=H$, which is a diagonal matrix whose entries are then sorted in increasing order. Furthermore, $\mathrm{SO}(2 k)$ has $k$ Cartan matrices $H_{q}$ with $q=1, \cdots, k$. The projection matrix (or just vector here) is $\alpha=\left\{\alpha_{i}\right\}$ given by solving the linear equations:

$$
\sum_{i=1}^{k} \alpha_{i} H_{i}=H
$$


and the decorated Dynkin diagrams are given by the vector $v=\alpha \cdot C_{\mathrm{SO}(2 k)}$. Each partition yields a different $H$ and therefore a different set of equations (A.4) and Dynkin labels $v$.

We should note that this analysis makes extensive use of the LieArt package of reference [82].

SO(8) example. To illustrate we work out an example with $\mathrm{SO}(8)$ in detail:

One partition of $\mathrm{SO}(8)$ is given by $[5,3]$. So the raising operator matrix is:

$$
X=\left(\begin{array}{cccccccc}
0 & 2 & 0 & 0 & 0 & 0 & 0 & 0 \\
0 & 0 & \sqrt{6} & 0 & 0 & 0 & 0 & 0 \\
0 & 0 & 0 & \sqrt{6} & 0 & 0 & 0 & 0 \\
0 & 0 & 0 & 0 & 2 & 0 & 0 & 0 \\
0 & 0 & 0 & 0 & 0 & 0 & 0 & 0 \\
0 & 0 & 0 & 0 & 0 & 0 & \sqrt{2} & 0 \\
0 & 0 & 0 & 0 & 0 & 0 & 0 & \sqrt{2} \\
0 & 0 & 0 & 0 & 0 & 0 & 0 & 0
\end{array}\right)
$$

and the corresponding Cartan matrix $H=\left[X, X^{\dagger}\right]=\operatorname{diag}(4,2,2,0,0,-2,-2,-4)$ after sorting out the entries.

The 4 Cartans of $\mathrm{SO}(8)$ are given by:

$$
\begin{aligned}
& H_{1}=\operatorname{diag}(1,-1,0,0,0,0,1,-1) \\
& H_{2}=\operatorname{diag}(0,1,-1,0,0,1,-1,0) \\
& H_{3}=\operatorname{diag}(0,0,1,-1,1,-1,0,0) \\
& H_{4}=\operatorname{diag}(0,0,1,1,-1,-1,0,0)
\end{aligned}
$$

where we are using the mathematician's conventions to be consistent with the use of the LieArt package.

The projection matrix $\alpha=\left(\alpha_{1}, \alpha_{2}, \alpha_{3}, \alpha_{4}\right)$ is then obtained by solving the equation:

$$
\alpha_{1} H_{1}+\alpha_{2} H_{2}+\alpha_{3} H_{3}+\alpha_{4} H_{4}=H
$$

which yields:

$$
\alpha=(4,6,4,4) \text {. }
$$

Thus given the Cartan matrix:

$$
C_{\mathrm{SO}(8)}=\left(\begin{array}{cccc}
2 & -1 & 0 & 0 \\
-1 & 2 & -1 & -1 \\
0 & -1 & 2 & 0 \\
0 & -1 & 0 & 2
\end{array}\right)
$$

the decorated Dynkin diagram specifies a vector $v=\alpha \cdot C_{\mathrm{SO}(8)}$ given by:

$$
v=(2,0,2,2)
$$

This procedure is repeated for every partition of $\mathrm{SO}(2 k)$ so as to obtain all of the necessary decorated Dynkin diagrams and projection matrices. 


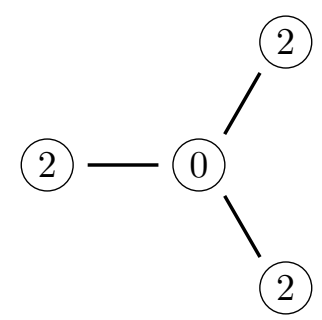

Figure 12. Decorated Dynkin diagram for the $[5,3]$ partition of $\mathrm{SO}(8)$.

\section{B From $6 \mathrm{D}$ to $4 \mathrm{D}$ conformal matter}

In this appendix we collect some features of $6 \mathrm{D}$ conformal matter and its compactification on a $T^{2}$. At long distances, this yields a $4 \mathrm{D} \mathcal{N}=2 \mathrm{SCFT}$. Here, we review both the scaling dimensions of Coulomb branch operators and the anomalies of these theories.

Coulomb branch operators. In this subsection we calculate the scaling dimension of the operators parameterizing the Coulomb branch. This data follows directly from the analysis of references [46-48]. Our main task here is to extract from this analysis the corresponding scaling dimensions. References $[46,47]$ implicitly give this information by showing that $4 \mathrm{D} \mathcal{N}=2(G, G)$ conformal matter is actually a compactification of a class $\mathcal{S}$ theory, specifying the corresponding Gaiotto curve as well. In reference [48] the corresponding Seiberg-Witten curve is obtained via the mirror to the elliptically fibered Calabi-Yau threefold of the F-theory background used to produce the 6D SCFT. Observe that F-theory compactified on a $T^{2}$ yields IIA on the same elliptic threefold, and mirror symmetry takes us to type IIB. The advantage of the IIB presentation is that now the Coulomb branch is parameterized in terms of the complex structure of this mirror geometry.

We opt to use the explicit Calabi-Yau geometries presented in reference [48]. To aid comparison with the results of this reference, we refer to the theory of $6 \mathrm{D}$ conformal matter with $(G, G)$ flavor symmetry given by $N$ M5-branes probing an ADE singularity as $\mathcal{T}(G, N)$. In this paper we focus exclusively on the case $N=1$.

We now use the results of reference [48] on the associated mirror geometries to compute the scaling dimensions of the Coulomb branch for the theories $\mathcal{T}\left(E_{6,7,8}, 1\right)$, on $T^{2}$. This method has been used before for $\mathcal{N}=2$ SCFTs, and is essentially adapted from the technique presented in reference [27].

The IIB mirror geometry for $\mathcal{T}\left(E_{6}, 1\right)$ on $T^{2}$ is given by the following local Calabi-Yau threefold:

$$
\left\{\begin{aligned}
f= & w^{2}+x_{1}^{3}+x_{2}^{2} \rho+\rho^{2}+\left(m_{1}+m_{1}^{\prime} y_{1}\right) x_{1} x_{2}^{2}+\left(m_{2}+m_{2}^{\prime} y_{1}\right) x_{1} x_{2}+\left(m_{3}+u_{1} y_{1}+m_{3}^{\prime} y_{1}^{2}\right) x_{2}^{2} \\
& +\left(m_{4}+u_{2} y_{1}+m_{4}^{\prime} y_{1}^{2}\right) x_{1}+\left(m_{5}+u_{3} y_{1}+m_{5}^{\prime} y_{1}^{2}\right) x_{2}+\left(m_{6}+u_{4} y_{1}+u_{5} y_{1}^{2}+m_{6}^{\prime} y_{1}^{3}\right)=0 \\
\rho= & \left(1+y_{1}+y_{2}\right), \\
x_{2}^{2}= & \rho .
\end{aligned}\right.
$$

where $y_{1}$ is a $\mathbb{C}^{*}$ coordinate, $x_{1}, x_{2}, w, \rho$ are complex coordinates, $m_{i}$ are general mass 
parameters and $u_{i}$ are the coulomb branch operator vevs,

$$
u_{i} \equiv\left\langle Z_{i}\right\rangle
$$

$f$ is a homogeneous polynomial in the complex coordinates and it scales as follows:

$$
f\left(\lambda^{a} x_{1}, \lambda^{b} x_{2}, \lambda^{c} \rho, \lambda^{d} w, y_{1}\right)=\lambda^{e} f\left(x_{1}, x_{2}, \rho, w, y_{1}\right) .
$$

The holomorphic three-form is defined as follows

$$
\Omega=\frac{d x_{1} \wedge d x_{2}}{w} \wedge \frac{d y_{1}}{y_{1}}
$$

By fixing the scale of $\Omega\left(\lambda^{a} x_{1}, \lambda^{b} x_{2}, \lambda^{c} \rho, \lambda^{d} w, y_{1}\right)=\lambda \Omega\left(x_{1}, x_{2}, \rho, w, y_{1}\right)$ to the unity, i.e. $[\Omega]=1$, the first four monomials of $f$ uniquely fix the other scalings

$$
\left[x_{1}\right]=a=4, \quad\left[x_{2}\right]=b=3, \quad[\rho]=c=[w]=d=6, \quad[f]=e=12 .
$$

Recalling that $y_{1}$ does not scale since it is just a phase, we obtain the scaling dimension of the Coulomb branch parameters,

$$
\left[u_{1}\right]=6, \quad\left[u_{2}\right]=8, \quad\left[u_{3}\right]=9, \quad\left[u_{4}\right]=\left[u_{5}\right]=12 .
$$

This agrees with the scaling dimensions of the Coulomb branch operators for the class $\mathcal{S}$ trinions with two minimal and one maximal puncture in [83].

The IIB mirror Calabi-Yau for $\mathcal{T}\left(E_{7}, 1\right)$ on $T^{2}$ is described by

$$
\left\{\begin{aligned}
f= & x_{1}^{2}+x_{2}^{3} \rho+\rho^{3}+\left(m_{1}+m_{1}^{\prime} y_{1}\right) x_{2} \rho^{2}+\left(m_{2}+u_{1} y_{1}+m_{2}^{\prime} y_{1}^{2}\right) \rho^{2} \\
& +\left(m_{3}+u_{2} y_{1}+m_{3}^{\prime} y_{1}^{2}\right) x_{2} \rho+\left(m_{4}+u_{3} y_{1}+m_{4}^{\prime} y_{1}^{2}\right) x_{2}^{2}+\left(m_{7}+u_{4} y_{1}+u_{5} y_{1}^{2}+m_{4}^{\prime} y_{1}^{3}\right) \rho \\
& +\left(m_{6}+u_{6} y_{1}+u_{7} y_{1}^{2}+m_{5}^{\prime} y_{1}^{3}\right) x_{2}+\left(m_{7}+u_{8} y_{1}+u_{9} y_{1}^{2}+u_{10} y_{1}^{3}+m_{7}^{\prime} y_{1}^{4}\right)=0 . \\
\rho= & \left(1+y_{1}+y_{2}\right) .
\end{aligned}\right.
$$

where again $y_{1}$ is a $\mathbb{C}^{*}$ coordinate, and $x_{1}, x_{2}, \rho$ are complex coordinates. The homogeneous polynomial $f$ scales as follows:

$$
f\left(\lambda^{a} x_{1}, \lambda^{b} x_{2}, \lambda^{c} \rho, y_{1}\right)=\lambda^{e} f\left(x_{1}, x_{2}, y_{1}\right) .
$$

The holomorphic three-form reads

$$
\Omega=\frac{d x_{2} \wedge d \rho}{x_{1}} \wedge \frac{d y_{1}}{y_{1}}
$$

and we impose that it scales like $[\Omega]=1$. The first three monomials again fix the scaling of the complex coordinates and of $f$ :

$$
\left[x_{1}\right]=a=9, \quad\left[x_{2}\right]=b=4, \quad[\rho]=c=6, \quad[f]=e=18 .
$$


By looking at the scaling of the other monomials involving the Coulomb branch vevs, the scaling dimensions of the Coulomb branch parameters are assigned

$$
\begin{array}{ll}
{\left[u_{1}\right]=6,} & {\left[u_{2}\right]=8,} \\
{\left[u_{3}\right]=10,} & {\left[u_{4}\right]=\left[u_{5}\right]=12,} \\
{\left[u_{6}\right]=\left[u_{7}\right]=14,} & {\left[u_{8}\right]=\left[u_{9}\right]=\left[u_{10}\right]=18 .}
\end{array}
$$

This agrees with the scaling dimensions of the Coulomb branch operators for the class $\mathcal{S}$ trinions with two minimal and one maximal puncture in [84].

The IIB mirror Calabi-Yau for $\mathcal{T}\left(E_{8}, 1\right)$ on $T^{2}$ is described by

$$
\left\{\begin{aligned}
f= & x_{1}^{2}+x_{2}^{3}+\rho^{5}+\left(m_{1}+m_{1}^{\prime} y_{1}\right) x_{2} \rho^{3}+\left(u y_{1}^{2}\right) \rho^{4}+\left(m_{2}+u_{1} y_{1}+m_{2}^{\prime} y_{1}^{2}\right) x_{2} \rho^{2} \\
& +\left(m_{3}+u_{2} y_{1}+u_{3} y_{1}^{2}+m_{3}^{\prime} y_{1}^{3}\right) \rho^{3}+\left(m_{4}+u_{4} y_{1}+u_{5} y_{1}^{2}+m_{4}^{\prime} y_{1}^{3}\right) x_{2} \rho \\
& +\left(m_{5}+u_{6} y_{1}+u_{7} y_{1}^{2}+u_{8} y_{1}^{3}+m_{5}^{\prime} y_{1}^{4}\right) \rho^{2}+\left(m_{6}+u_{9} y_{1}+u_{10} y_{1}^{2}+u_{11} y_{1}^{3}+m_{6}^{\prime} y_{1}^{4}\right) x_{2} \\
& +\left(m_{7}+u_{12} y_{1}+u_{13} y_{1}^{2}+u_{14} y_{1}^{3}+u_{15} y_{1}^{4}+m_{7}^{\prime} y_{1}^{5}\right) \rho \\
& +\left(m_{8}+u_{16} y_{1}+u_{17} y_{1}^{2}+u_{18} y_{1}^{3}+u_{19} y_{1}^{4}+u_{20} y_{1}^{5}+m_{8}^{\prime} y_{1}^{6}\right)=0 \\
\rho= & \left(1+y_{1}+y_{2}\right) .
\end{aligned}\right.
$$

where again $y_{1}$ is a $\mathbb{C}^{*}$ coordinate, and the $x_{1}, x_{2}, \rho$ are complex coordinates. The homogeneous polynomial $f$ scales as in equation (B.6). The holomorphic three-form is analogous to the $E_{7}$ case, (B.7). By imposing $[\Omega]=1$, the first three monomials of $f$ fix the scaling of the coordinates,

$$
\left[x_{1}\right]=a=15, \quad\left[x_{2}\right]=b=10, \quad[\rho]=c=6, \quad[f]=e=30 .
$$

The other monomials involving the Coulomb branch vevs automatically assign the following scaling dimensions

$$
\begin{aligned}
{[u] } & =6, & & {\left[u_{1}\right]=8, } \\
{\left[u_{2}\right] } & =\left[u_{3}\right]=12, & {\left[u_{4}\right] } & =\left[u_{5}\right]=14, \\
{\left[u_{6}\right] } & =\left[u_{7}\right]=\left[u_{8}\right]=18, & {\left[u_{9}\right] } & =\left[u_{10}\right]=\left[u_{11}\right]=20, \\
{\left[u_{12}\right] } & =\left[u_{13}\right]=\left[u_{14}\right]=\left[u_{15}\right]=24, & {\left[u_{16}\right] } & =\left[u_{17}\right]=\left[u_{18}\right]=\left[u_{19}\right]=\left[u_{20}\right]=30 .
\end{aligned}
$$

This agrees with the scaling dimensions of the Coulomb branch operators for the class $\mathcal{S}$ trinions with two minimal and one maximal puncture in [85].

Finally, for the $D_{k}$ conformal matter theories $\mathcal{T}(\mathrm{SO}(2 k), 1)$ with $k>2$ on $T^{2}$ the scaling dimensions of the Coulomb branch operators can be read off in a similar way from the curve (5.4) in [46].

Anomaly polynomials. Given the importance of the UV anomalies we now review how they were obtained in table 5 . When studying an M5-brane probing $D$ - and $E$ type singularities we obtain $6 \mathrm{D}$ SCFTs also called $(G, G) 6 \mathrm{D}$ conformal matter with 


\begin{tabular}{|c|c|c|c|c|}
\hline$(G, G)$ & $\left(D_{k}, D_{k}\right)$ & $\left(E_{6}, E_{6}\right)$ & $\left(E_{7}, E_{7}\right)$ & $\left(E_{8}, E_{8}\right)$ \\
\hline $24 \alpha$ & $10 k^{2}-57 k+81$ & 319 & 1670 & 12489 \\
\hline $48 \beta$ & $-\left(2 k^{2}-3 k-9\right)$ & -89 & -250 & -831 \\
\hline$\frac{5760}{7} \gamma$ & $k(2 k-1)+1$ & 79 & 134 & 249 \\
\hline$\frac{5760}{4} \delta$ & $-(k(2 k-1)+1)$ & -79 & -134 & -249 \\
\hline $24 \kappa_{L}=24 \kappa_{R}$ & $2 k-2$ & 12 & 18 & 30 \\
\hline
\end{tabular}

Table 8. Coefficients of 6D anomaly polynomial (B.12).

anomaly polynomial:

$$
\begin{aligned}
I_{8}= & \alpha c_{2}\left(R_{6 D}\right)^{2}+\beta c_{2}\left(R_{6 D}\right) p_{1}(T)+\gamma p_{1}(T)^{2}+\delta p_{2}(T)+\kappa_{L} p_{1}(T) \frac{\operatorname{Tr}\left(F_{L}^{2}\right)}{4} \\
& +\kappa_{R} p_{1}(T) \frac{\operatorname{Tr}\left(F_{R}^{2}\right)}{4}+\ldots
\end{aligned}
$$

where the explicit expression for the $6 \mathrm{D}$ anomaly polynomial coefficients were computed in [86], and are listed in table 8.

In order to obtain a $4 \mathrm{D} \mathcal{N}=2 \mathrm{SCFT}$, we compactify these theories on $T^{2}$ and consider the general anomaly polynomial for a $4 \mathrm{D}$ theory

$$
\begin{aligned}
I_{6}= & \frac{k_{R R R}}{6} c_{1}(R)^{3}-\frac{k_{R}}{24} p_{1}(T) c_{1}(R)+k_{R G_{L} G_{L}} \frac{\operatorname{Tr}\left(F_{G_{L}}^{2}\right)}{4} c_{1}(R) \\
& +k_{R G_{R} G_{R}} \frac{\operatorname{Tr}\left(F_{G_{R}}^{2}\right)}{4} c_{1}(R)+\ldots,
\end{aligned}
$$

where $R=R_{\mathrm{UV}}$ is the R-symmetry of the $\mathrm{UV} \mathcal{N}=2 \mathrm{SCFT}$, viewed as an $\mathcal{N}=1 \mathrm{SCFT}$, $T$ is the formal tangent bundle, $F$ is the field strength of $G_{L}$ or $G_{R}$ flavor symmetries, and the dots indicate possible abelian flavor symmetries and mixed contributions. Moreover we have the following relations

$$
\operatorname{Tr}\left(R^{3}\right)=k_{R R R}, \quad \operatorname{Tr}(R)=k_{R}, \quad \operatorname{Tr}\left(R F_{G_{L, R}}^{A} F_{G_{L, R}}^{B}\right)=-\frac{k_{R G_{L, R} G_{L, R}}}{2} \delta^{A B} .
$$

From them, the definition of $R$, and

$$
\operatorname{Tr}\left(R_{\mathcal{N}=2} F_{G_{L, R}}^{A} F_{G_{L, R}}^{B}\right)=-\frac{k_{L, R}}{2} \delta^{A B}
$$

we read off the anomalies

$$
\begin{aligned}
a_{\mathrm{UV}} & =\frac{9}{32} k_{R R R}-\frac{3}{32} k_{R} \\
c_{\mathrm{UV}} & =\frac{9}{32} k_{R R R}-\frac{5}{32} k_{R} \\
k_{L} & =3 k_{R G_{L} G_{L}} \\
k_{R} & =3 k_{R G_{R} G_{R}} .
\end{aligned}
$$


In terms of the $6 \mathrm{D}$ anomaly polynomial coefficients [46, 47], we finally identify

$$
\begin{aligned}
a_{\mathrm{UV}} & =24 \gamma-12 \beta-18 \delta \\
c_{\mathrm{UV}} & =64 \gamma-12 \beta-8 \delta \\
k_{L} & =48 \kappa_{L} \\
k_{R} & =48 \kappa_{R} .
\end{aligned}
$$

Once evaluated at the values of table 8 the above equations yield exactly the UV values of table 5 , as expected.

\section{Accessing the complete tables}

Included with the arXiv submission is a set of Mathematica scripts which can be used to access the full set of theories generated by nilpotent deformations of the $\mathcal{N}=2$ theories considered in this paper. Indeed, due to the rather large size of the dataset it is impractical to list all of our results in the format of a paper.

Instead we have written a Mathematica script which outputs the complete list of all possible nilpotent deformations for the theories described above. The necessary files are attached to this paper (see the online "Supplementary Material" section) and on the arXiv page (to access them there, first proceed to the arXiv abstract page for this paper. On the righthand side, there is a box with the title "Download." Click on "Other formats" and then download the source files for the arXiv submission).

To access the full database, one simply needs to download the following six files and store them in the same folder: "ProbeD3brane.m", "ConformalMatter.m", "ProbeD3braneFlavorK.m", "ConformalMatterFlavorK.m", "NilpotentDeformations.m", "Results.nb". Essentially, the first file contains all of the information for nilpotent deformations of the probe D3-brane theories (with and without flipper field deformations), except for the flavor central charge. The second file stores all of the information for the nilpotent deformations of 4D conformal matter (with and without flipper field deformations), except for the flavor central charge. The next two files contain all of the information about the flavor central charges for the Minahan-Nemeshansky and conformal matter theories respectively. The file "NilpotentDeformations.m" does all of the formatting, and finally the code "Results.nb" loads the previous three packages and outputs the results. Thus the only file the user needs to run and worry about is the last one: "Results.nb". When running this file the user is provided with a list of options:

1. First one can choose between the four kinds of deformations: probe D3-brane theories with plain mass deformations, probe D3-brane theories with flipper field deformations, 4D conformal matter with plain mass deformations, and 4D conformal matter with flipper field deformations.

2. Secondly one can choose between the $a_{\mathrm{IR}}, c_{\mathrm{IR}}$ anomalies and operator scaling dimensions or the tables with the flavor central charges. 
3. Then the user should select the flavor groups: $D_{4}, E_{6}, E_{7}$, or $E_{8}$ for deformations of the probe D3-brane theories, and $\left(D_{4}, D_{4}\right),\left(E_{6}, E_{6}\right),\left(E_{7}, E_{7}\right)$, or $\left(E_{8}, E_{8}\right)$ for deformations of $4 \mathrm{D}$ conformal matter.

4. If a probe D3-brane theory is selected then the user can choose from two options:

(a) select a single deformation by choosing the Bala-Carter label (or partition of $D_{4}$ ) of the flavor group from the provided popup menu below.

(b) select the whole table.

5. If instead a $4 \mathrm{D}$ conformal matter theory is selected the user has three options:

(a) select a single deformation chosen by selecting the left and right Bala-Carter labels (or partitions of $D_{4}$ ) for the breaking of the left and right flavors.

(b) select all of the deformations with a given left (or right) deformation, by selecting a single Bala-Carter label (or partition of $D_{4}$ ).

(c) select the whole table.

6. The resulting table is then outputted. We also provide for the probe D3-brane theories the branching rules from the adjoint of $G$ to the $\mathrm{SU}(2)$ irreps for the selected deformations.

Finally, due to the form of the general equations used to compute the central charges it is clear that all of our results are algebraic numbers. However not all are rational. To differentiate the two in the tables we list the rational values exactly (by keeping their rational form) while we only give numerical values for the ones with irrational central charges.

For the convenience of the reader, in the following subsections we list the explicit tables for all of the nilpotent deformations of the probe D3-brane theory with $\mathrm{SO}(8)$ flavor symmetry, but only the rational theories for the other nilpotent networks.

As a point of notation, here we make reference to $K_{\mathrm{IR}}$ as well as $k_{\mathrm{IR}}$. 
Nilpotent network for $\mathrm{SU}(2)$ with four flavors

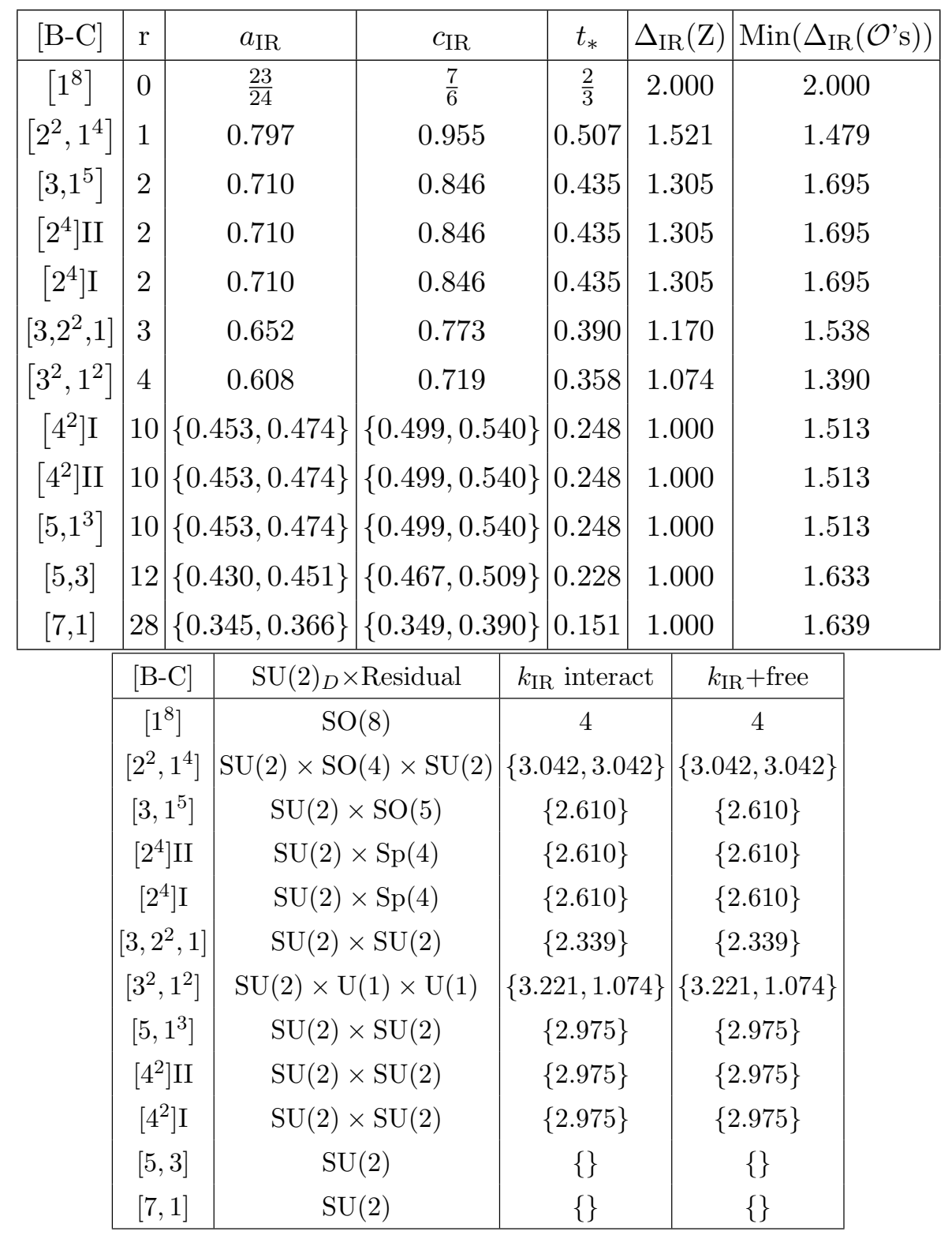

Table 9. Plain nilpotent deformations of the probe D3-brane theory with $D_{4}$ flavor symmetry. The top table has the central charges $a_{\mathrm{IR}}$ and $c_{\mathrm{IR}}$ as well as scaling dimensions while the table below contains the information about the flavor central charges. 


\begin{tabular}{|c|c|c|c|c|c|c|c|}
\hline$[\mathrm{B}-\mathrm{C}]$ & $\mathrm{r}$ & $a_{\mathrm{IR}}$ & \multicolumn{2}{|l|}{$c_{\mathrm{IR}}$} & $t_{*}$ & $\Delta_{\mathrm{IR}}(\mathrm{Z})$ & $\operatorname{Min}\left(\Delta_{\mathrm{IR}}\left(\mathcal{O}^{\prime} \mathrm{s}\right)\right.$ \\
\hline$\left[1^{8}\right]$ & 0 & $\left\{\frac{23}{24}, \frac{37}{24}\right\}$ & $\left\{\frac{7}{6}, \frac{7}{3}\right\}$ & & $\frac{2}{3}$ & 2.00 & 2.000 \\
\hline$\left[2^{2}, 1^{4}\right]$ & 1 & $\{0.962,1.358\}$ & $\{1.267,2.0$ & $058\}$ & 0.459 & 1.37 & 1.623 \\
\hline$\left[3,1^{5}\right]$ & 2 & $\{0.809,1.267\}$ & $\{1.020,1.9$ & $936\}$ & 0.376 & 1.12 & 1.872 \\
\hline$\left[2^{4}\right] \mathrm{II}$ & 2 & $\{0.809,1.267\}$ & $\{1.020,1.9$ & $936\}$ & 0.376 & 1.12 & 1.872 \\
\hline$\left[2^{4}\right] \mathrm{I}$ & 2 & $\{0.809,1.267\}$ & $\{1.020,1.9$ & $936\}$ & 0.376 & 1.12 & 1.872 \\
\hline$\left[3,2^{2}, 1\right]$ & 3 & $\{0.728,1.207\}$ & $\{0.911,1.8$ & $369\}$ & 0.344 & 1.03 & 1.709 \\
\hline$\left[3^{2}, 1^{2}\right]$ & 4 & $\left\{\frac{7}{12}, \frac{7}{6}\right\}$ & $\left\{\frac{2}{3}, \frac{11}{6}\right\}$ & & $\frac{1}{3}$ & 1.00 & 1.500 \\
\hline$\left[5,1^{3}\right]$ & 10 & $\left\{\frac{11}{24}, \frac{25}{24}\right\}$ & $\left\{\frac{1}{2}, \frac{5}{3}\right\}$ & & $\frac{2}{9}$ & 1.00 & 1.667 \\
\hline$\left[4^{2}\right] \mathrm{II}$ & 10 & $\left\{\frac{11}{24}, \frac{25}{24}\right\}$ & $\left\{\frac{1}{2}, \frac{5}{3}\right\}$ & & $\frac{2}{9}$ & 1.00 & 1.667 \\
\hline$\left[4^{2}\right] \mathrm{I}$ & 10 & $\left\{\frac{11}{24}, \frac{25}{24}\right\}$ & $\left\{\frac{1}{2}, \frac{5}{3}\right\}$ & & $\frac{2}{9}$ & 1.0 & 1.667 \\
\hline$[5,3]$ & 12 & $\left\{\frac{6349}{13872}, \frac{1769}{1734}\right\}$ & $\left\{\frac{3523}{6936}, \frac{566}{346}\right.$ & & $\frac{10}{51}$ & 1.0 & 1.824 \\
\hline$[7,1]$ & 28 & $\left\{\frac{43}{120}, \frac{113}{120}\right\}$ & $\left\{\frac{11}{30}, \frac{23}{15}\right.$ & & $\frac{2}{15}$ & 1.0 & 1.800 \\
\hline \multicolumn{2}{|c|}{$[\mathrm{B}-\mathrm{C}]$} & \multicolumn{2}{|c|}{$\mathrm{SU}(2)_{D} \times$ Residual } & \multicolumn{3}{|c|}{$k_{\mathrm{IR}}$ interact } & $k_{\mathrm{IR}}+$ free \\
\hline \multicolumn{2}{|c|}{$\left[1^{8}\right]$} & \multicolumn{2}{|c|}{$\mathrm{SO}(8)$} & \multicolumn{3}{|c|}{4} & 16 \\
\hline \multicolumn{2}{|c|}{$\left[2^{2}, 1^{4}\right]$} & \multicolumn{2}{|c|}{$\mathrm{SU}(2) \times \mathrm{SO}(4) \times \mathrm{SU}(2)$} & \multicolumn{3}{|c|}{$6.490,6.490$} & $10.491,10.491\}$ \\
\hline \multicolumn{2}{|c|}{$\left[3,1^{5}\right]$} & \multicolumn{2}{|c|}{$\mathrm{SU}(2) \times \mathrm{SO}(5)$} & \multicolumn{3}{|c|}{3.745} & $\{9.745\}$ \\
\hline \multicolumn{2}{|c|}{$\left[2^{4}\right] \mathrm{II}$} & \multicolumn{2}{|c|}{$\mathrm{SU}(2) \times \mathrm{Sp}(4)$} & \multicolumn{3}{|c|}{3.745} & $\{9.745\}$ \\
\hline \multicolumn{2}{|c|}{$\left[2^{4}\right] \mathrm{I}$} & \multicolumn{2}{|c|}{$\mathrm{SU}(2) \times \mathrm{Sp}(4)$} & & $\{3.745\}$ & & $\{9.745\}$ \\
\hline$[3,2$ & $\left.{ }^{2}, 1\right]$ & $\mathrm{SU}(2) \times$ & $\mathrm{SU}(2)$ & & $\{2.484\}$ & & $\{8.484\}$ \\
\hline$\left[3^{2}\right.$ & & $\mathrm{SU}(2) \times \mathrm{U}(1$ & $\times U(1)$ & & $\{3,1\}$ & & $\{9,3\}$ \\
\hline$[5$, & & $\mathrm{SU}(2) \times$ & $\mathrm{SU}(2)$ & & $\left\{\frac{8}{3}\right\}$ & & $\left\{\frac{32}{3}\right\}$ \\
\hline$\left[4^{2}\right.$ & & $\mathrm{SU}(2) \times$ & $\mathrm{SU}(2)$ & & $\left\{\frac{8}{3}\right\}$ & & $\left\{\frac{32}{3}\right\}$ \\
\hline$[4$ & & $\mathrm{SU}(2) \times$ & $\mathrm{SU}(2)$ & & $\left\{\frac{8}{3}\right\}$ & & $\left\{\frac{32}{3}\right\}$ \\
\hline$[5$ & & $\mathrm{SU}(2$ & & & \{\} & & \{\} \\
\hline$[7$ & & $\mathrm{SU}(2$ & & & \{\} & & \{\} \\
\hline
\end{tabular}

Table 10. Flipper field deformations of the probe D3-brane theory with $D_{4}$ flavor. The top table has the central charges $a_{\mathrm{IR}}$ and $c_{\mathrm{IR}}$ as well as scaling dimensions while the table below contains the information about the flavor central charges. The cyan highlighted entries align with the $H_{0}, H_{1}$ and $H_{2}$ Argyres-Douglas theories, as first noted in $[16,17]$. The other rational entry with partition $[5,3]$ also aligns with [18]. 
Tables of rational theories: Minahan-Nemeschansky theories

\begin{tabular}{|c|c|c|c|c|c|c|c|}
\hline$[\mathrm{B}-\mathrm{C}]$ & $\mathrm{r}$ & $a_{\mathrm{IR}}$ & $c_{\mathrm{IR}}$ & $t_{*}$ & $\Delta_{\mathrm{IR}}(\mathrm{Z})$ & $\operatorname{Min}\left(\Delta_{\mathrm{IR}}(\mathcal{O} ' \mathrm{~s})\right)$ \\
\hline 0 & 0 & $\frac{41}{24}$ & $\frac{13}{6}$ & $\frac{2}{3}$ & 3.000 & \multicolumn{2}{|c|}{2.000} \\
$A_{2}+2 A_{1}$ & 6 & $\frac{97}{96}$ & $\frac{119}{96}$ & $\frac{1}{3}$ & 1.500 & \multicolumn{2}{|c|}{1.500} \\
\hline$[\mathrm{B}-\mathrm{C}]$ & $\mathrm{SU}(2)_{D} \times$ Residual & $k_{\mathrm{IR}}$ interact & $k_{\mathrm{IR}}+$ free \\
\hline 0 & \multicolumn{5}{|c|}{$E_{6}$} & 6 & 6 \\
$A_{2}+2 A_{1}$ & $\mathrm{SU}(2) \times \mathrm{SU}(2) \times \mathrm{U}(1)$ & $\{18,18\}$ & $\{18,18\}$ \\
\hline
\end{tabular}

Table 11. Plain nilpotent mass deformations of the Minahan-Nemeschansky theory with $E_{6}$ flavor. The top table has the central charges $a_{\mathrm{IR}}$ and $c_{\mathrm{IR}}$ as well as scaling dimensions while the table below contains the information about the flavor central charges.

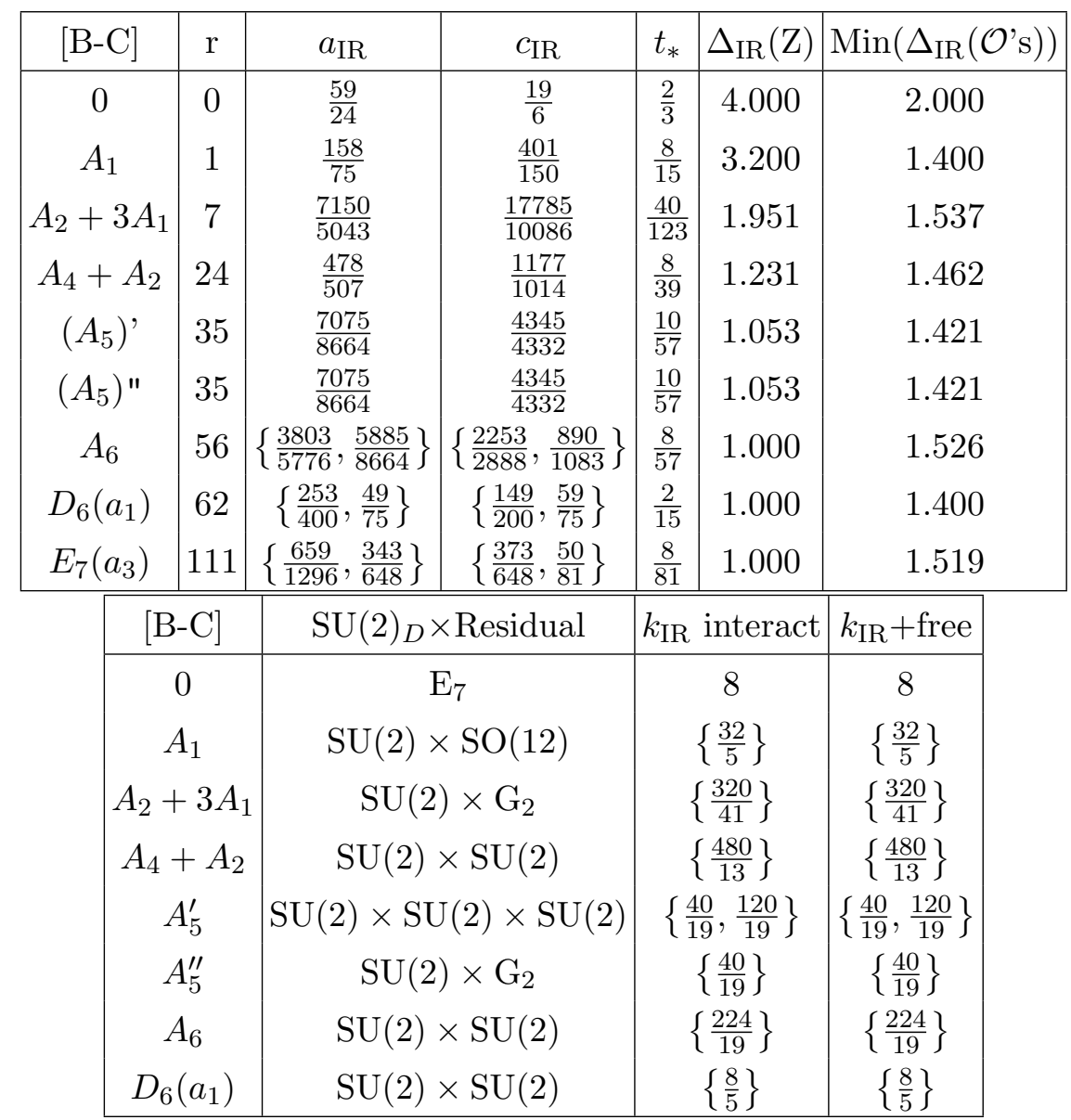

Table 12. Plain nilpotent mass deformations of the Minahan-Nemeschansky theory with $E_{7}$ flavor, only rational values. The top table has the central charges $a_{\mathrm{IR}}$ and $c_{\mathrm{IR}}$ as well as scaling dimensions while the table below contains the information about the flavor central charges. 


\begin{tabular}{|c|c|c|c|c|c|c|}
\hline$[\mathrm{B}-\mathrm{C}]$ & $\mathrm{r}$ & $a_{\mathrm{IR}}$ & $c_{\mathrm{IR}}$ & $t_{*}$ & $\Delta_{\mathrm{IR}}(\mathrm{Z})$ & $\operatorname{Min}\left(\Delta_{\mathrm{IR}}\left(\mathcal{O}^{\prime} \mathrm{s}\right)\right)$ \\
\hline 0 & 0 & $\frac{95}{24}$ & $\frac{31}{6}$ & $\frac{2}{3}$ & 6. & 2. \\
$A_{2}+3 A_{1}$ & 7 & $\frac{223}{96}$ & $\frac{281}{96}$ & $\frac{1}{3}$ & 3. & 1.5 \\
$E_{8}\left(a_{1}\right)$ & 760 & $\left\{\frac{5471}{13872}, \frac{120}{289}\right\}$ & $\left\{\frac{2897}{6936}, \frac{531}{1156}\right\}$ & $\frac{2}{51}$ & 1. & 1.58824 \\
\hline
\end{tabular}

Table 13. Plain nilpotent mass deformations of the Minahan-Nemeschansky theory with $E_{8}$ flavor, only rational values. The top table has the central charges $a_{\mathrm{IR}}$ and $c_{\mathrm{IR}}$ as well as scaling dimensions while the table below contains the information about the flavor central charges.

\begin{tabular}{|c|c|c|c|c|c|c|}
\hline$[\mathrm{B}-\mathrm{C}]$ & $\mathrm{r}$ & $a_{\mathrm{IR}}$ & $c_{\mathrm{IR}}$ & $t_{*}$ & $\Delta_{\mathrm{IR}}(\mathrm{Z})$ & $\operatorname{Min}\left(\Delta_{\mathrm{IR}}\left(\mathcal{O}^{\prime} \mathrm{s}\right)\right)$ \\
\hline 0 & 0 & $\left\{\frac{41}{24}, \frac{10}{3}\right\}$ & $\left\{\frac{13}{6}, \frac{65}{12}\right\}$ & $\frac{2}{3}$ & 3.000 & 2.000 \\
$D_{4}$ & 28 & $\left\{\frac{7}{12}, \frac{53}{24}\right\}$ & $\left\{\frac{2}{3}, \frac{47}{12}\right\}$ & $\frac{1}{6}$ & 1.000 & 1.500 \\
$D_{5}$ & 60 & $\left\{\frac{11}{24}, \frac{25}{12}\right\}$ & $\left\{\frac{1}{2}, \frac{15}{4}\right\}$ & $\frac{1}{9}$ & 1.000 & 1.667 \\
$E_{6}$ & 156 & $\left\{\frac{43}{120}, \frac{119}{60}\right\}$ & $\left\{\frac{11}{30}, \frac{217}{60}\right\}$ & $\frac{1}{15}$ & 1.000 & 1.800 \\
\hline \multicolumn{6}{|c|}{} \\
\cline { 2 - 6 } & {$[\mathrm{B}-\mathrm{C}]$} & $\mathrm{SU}(2)_{D} \times$ Residual & $k_{\mathrm{IR}}$ interact & $k_{\mathrm{IR}}+$ free \\
\cline { 2 - 6 } & 0 & \multicolumn{2}{|c|}{$\mathrm{E}_{6}$} & 6 & 30 \\
& $D_{4}$ & $\mathrm{SU}(2) \times \mathrm{SU}(3)$ & $\{3\}$ & $\{15\}$ \\
$D_{5}$ & $\mathrm{SU}(2) \times \mathrm{U}(1)$ & $\{6\}$ & $\{24\}$ \\
\cline { 2 - 5 }
\end{tabular}

Table 14. Flipper field deformations of the Minahan-Nemeschansky theory with $E_{6}$ flavor, only rational values. The top table has the central charges $a_{\mathrm{IR}}$ and $c_{\mathrm{IR}}$ as well as scaling dimensions while the table below contains the information about the flavor central charges. The cyan highlighted entries align with the $H_{0}, H_{1}$ and $H_{2}$ Argyres-Douglas theories, as first noted in [17, 18]. 


\begin{tabular}{|c|c|c|c|c|c|c|}
\hline$[\mathrm{B}-\mathrm{C}]$ & $\mathrm{r}$ & $a_{\mathrm{IR}}$ & $c_{\mathrm{IR}}$ & $t_{*}$ & $\Delta_{\mathrm{IR}}(\mathrm{Z})$ & $\operatorname{Min}\left(\Delta_{\mathrm{IR}}\left(\mathcal{O}^{\prime} \mathrm{s}\right)\right)$ \\
\hline 0 & 0 & $\left\{\frac{59}{24}, \frac{251}{48}\right\}$ & $\left\{\frac{19}{6}, \frac{209}{24}\right\}$ & $\frac{2}{3}$ & 4.000 & 2.000 \\
$A_{2}+3 A_{1}$ & 7 & $\left\{\frac{1263}{8214}, \frac{143899}{32856}\right\}$ & $\left\{\frac{121465}{65712}, \frac{466453}{65712}\right\}$ & $\frac{31}{111}$ & 1.676 & 1.743 \\
$E_{6}$ & 156 & $\left\{\frac{11}{24}, \frac{155}{48}\right\}$ & $\left\{\frac{1}{2}, \frac{145}{24}\right\}$ & $\frac{2}{27}$ & 1.000 & 1.667 \\
$E_{7}$ & 399 & $\left\{\frac{43}{120}, \frac{751}{240}\right\}$ & $\left\{\frac{11}{30}, \frac{709}{120}\right\}$ & $\frac{2}{45}$ & 1.000 & 1.800 \\
\hline
\end{tabular}

\begin{tabular}{|c|c|c|c|}
\hline \hline$[\mathrm{B}-\mathrm{C}]$ & $\mathrm{SU}(2)_{D} \times$ Residual & $k_{\mathrm{IR}}$ interact & $k_{\mathrm{IR}}+$ free \\
\hline 0 & $\mathrm{E}_{7}$ & 8 & 44 \\
$A_{2}+3 A_{1}$ & $\mathrm{SU}(2) \times \mathrm{G}_{2}$ & $\left\{\frac{284}{37}\right\}$ & $\left\{\frac{1246}{37}\right\}$ \\
$E_{6}$ & $\mathrm{SU}(2) \times \mathrm{SU}(2)$ & $\left\{\frac{8}{3}\right\}$ & $\left\{\frac{44}{3}\right\}$ \\
\hline
\end{tabular}

Table 15. Flipper field deformations of the Minahan-Nemeschansky theory with $E_{7}$ flavor, only rational values. The top table has the central charges $a_{\mathrm{IR}}$ and $c_{\mathrm{IR}}$ as well as scaling dimensions while the table below contains the information about the flavor central charges. The cyan highlighted entries align with the $H_{0}$ and $H_{1}$ Argyres-Douglas theories, as first noted in [17]. Compared with reference [17], we also find an additional flipper field deformation which yields the $H_{1}$ theory for the $E_{6}$ Bala-Carter label, with embedding index $r=156$. The other rational central charges are also in agreement with [87].

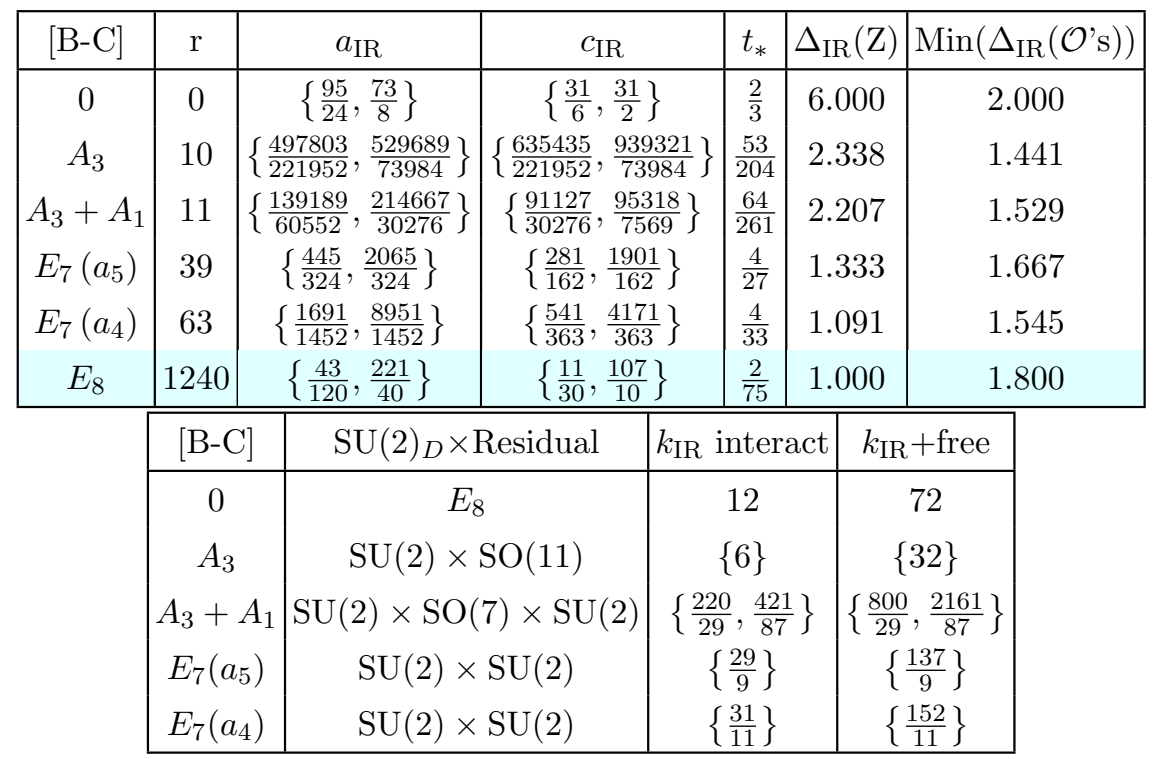

Table 16. Flipper field deformations of the Minahan-Nemeschansky theory with $E_{8}$ flavor, only rational values. The top table has the central charges $a_{\mathrm{IR}}$ and $c_{\mathrm{IR}}$ as well as scaling dimensions while the table below contains the information about the flavor central charges. The cyan highlighted entry aligns with the $H_{0}$ Argyres-Douglas theory, as first noted in [17]. The other rational central charges are also in agreement with [87]. 
Tables of rational theories: conformal matter

\begin{tabular}{|c|c|c|c|c|c|c|c|}
\hline$[\mathrm{B}-\mathrm{C}]_{L}$ & {$[\mathrm{~B}-\mathrm{C}]_{R}$} & $r_{L}$ & $r_{R}$ & $r_{L}+r_{R}$ & $a_{\mathrm{IR}}$ & $c_{\mathrm{IR}}$ & $t_{*}$ \\
\hline 0 & 0 & 0 & 0 & 0 & $\frac{613}{24}$ & $\frac{173}{6}$ & $\frac{2}{3}$ \\
$2 A_{2}+A_{1}$ & $2 A_{2}$ & 9 & 8 & 17 & $\frac{68050}{4107}$ & $\frac{150715}{8214}$ & $\frac{40}{111}$ \\
$A_{5}$ & $2 A_{2}+A_{1}$ & 35 & 9 & 44 & $\left\{\frac{316}{25}, \frac{3817}{300}\right\}$ & $\left\{\frac{346}{25}, \frac{2101}{150}\right\}$ & $\frac{4}{15}$ \\
\hline \hline$[\mathrm{B}-\mathrm{C}]_{L}$ & {$[\mathrm{~B}-\mathrm{C}]_{R}$} & $t_{*}$ & $\operatorname{Min}\left(\Delta_{\mathrm{IR}}(\mathrm{Z} ' \mathrm{~s})\right)$ & $\operatorname{Min}\left(\Delta_{\mathrm{IR}}\left(\mathcal{O}_{L}{ }^{\prime} \mathrm{s}\right)\right)$ & $\operatorname{Min}\left(\Delta_{\mathrm{IR}}\left(\mathcal{O}_{R}{ }^{\prime} \mathrm{s}\right)\right)$ \\
0 & 0 & $\frac{2}{3}$ & 6.000 & 2.000 & 2.000 \\
$2 A_{2}+A_{1}$ & $2 A_{2}$ & $\frac{40}{111}$ & 3.243 & 1.108 & 1.378 \\
$A_{5}$ & $2 A_{2}+A_{1}$ & $\frac{4}{15}$ & 2.400 & 1.000 & 1.600 \\
\hline
\end{tabular}

Table 17. Plain nilpotent mass deformations of $\left(E_{6}, E_{6}\right)$ conformal matter, only rational values.

\begin{tabular}{|c|c|c|c|c|c|c|c|c|}
\hline$[\mathrm{B}-\mathrm{C}]_{L}$ & {$[\mathrm{~B}-\mathrm{C}]_{R}$} & $r_{L}$ & $r_{R}$ & $r_{L}+r_{R}$ & $a_{\mathrm{IR}}$ & & $c_{\mathrm{IR}}$ & $t_{*}$ \\
\hline 0 & 0 & 0 & 0 & 0 & $\frac{817}{12}$ & & $\frac{221}{3}$ & $\frac{2}{3}$ \\
\hline$D_{4}+A_{1}$ & $D_{4}+A_{1}$ & 29 & 29 & 58 & $\left\{\frac{314941}{8400}, \frac{105097}{2800}\right\}$ & & $\left.\frac{47843}{1200}, \frac{15981}{400}\right\}$ & $\frac{31}{105}$ \\
\hline$D_{5}$ & $\left(3 A_{1}\right)^{\prime \prime}$ & 60 & 3 & 63 & $\left\{\frac{233959}{6272}, \frac{235135}{6272}\right\}$ & & $\left.\frac{247315}{6272}, \frac{249667}{6272}\right\}$ & $\frac{13}{42}$ \\
\hline$D_{5}$ & $\left(3 A_{1}\right)^{\prime}$ & 60 & 3 & 63 & $\left\{\frac{233959}{6272}, \frac{235135}{6272}\right\}$ & & $\left.\frac{247315}{6272}, \frac{249667}{6272}\right\}$ & $\frac{13}{42}$ \\
\hline$D_{5}+A_{1}$ & 0 & 61 & 0 & 61 & $\left\{\frac{63612}{1681}, \frac{1022835}{26896}\right\}$ & & $\left.\frac{38047}{13448}, \frac{271545}{6724}\right\}$ & $\frac{13}{41}$ \\
\hline$D_{5}+A_{1}$ & $D_{4}\left(a_{1}\right)$ & 61 & 12 & 73 & $\left\{\frac{27729}{784}, \frac{6969}{196}\right\}$ & & $\left.\frac{3663}{98}, \frac{14799}{392}\right\}$ & $\frac{2}{7}$ \\
\hline$D_{5}+A_{1}$ & $A_{3}+2 A_{1}$ & 61 & 12 & 73 & $\left\{\frac{27729}{784}, \frac{6969}{196}\right\}$ & & $\left\{\frac{3663}{98}, \frac{14799}{392}\right\}$ & $\frac{2}{7}$ \\
\hline$E_{6}\left(a_{1}\right)$ & $A_{3}$ & 84 & 10 & 94 & $\left\{\frac{1583}{48}, \frac{199}{6}\right\}$ & & $\left\{\frac{4177}{120}, \frac{2111}{60}\right\}$ & $\frac{4}{15}$ \\
\hline$E_{6}$ & $A_{3}$ & 156 & 10 & 166 & $\left\{\frac{995}{36}, \frac{1999}{72}\right\}$ & & $\left\{\frac{1049}{36}, \frac{529}{18}\right\}$ & $\frac{2}{9}$ \\
\hline$E_{7}\left(a_{1}\right)$ & $A_{2}$ & 231 & 4 & 235 & $\left\{\frac{2992009}{121104}, \frac{187789}{7569}\right\}$ & & $\left.\frac{576001}{60552}, \frac{198577}{7569}\right\}$ & $\frac{52}{261}$ \\
\hline$E_{7}\left(a_{1}\right)$ & $4 A_{1}$ & 231 & 4 & 235 & $\left\{\frac{2992009}{121104}, \frac{187789}{7569}\right\}$ & & $\left.\frac{576001}{60552}, \frac{198577}{7569}\right\}$ & $\frac{52}{261}$ \\
\hline$[\mathrm{B}-\mathrm{C}]_{L}$ & {$[\mathrm{~B}-\mathrm{C}]_{R}$} & $t_{*}$ & \multicolumn{2}{|c|}{$\operatorname{Min}\left(\Delta_{\mathrm{IR}}\left(\mathrm{Z}^{\prime} \mathrm{s}\right)\right)$} & \multicolumn{2}{|c|}{$\operatorname{Min}\left(\Delta_{\mathrm{IR}}\left(\mathcal{O}_{L}{ }^{\prime} \mathrm{s}\right)\right)$} & \multicolumn{2}{|c|}{$\operatorname{Min}\left(\Delta_{\mathrm{IR}}\left(\mathcal{O}_{R}{ }^{\prime} \mathrm{s}\right)\right)$} \\
\hline 0 & 0 & $\frac{2}{3}$ & \multicolumn{2}{|r|}{6.000} & \multicolumn{2}{|l|}{2.000} & \multicolumn{2}{|l|}{2.000} \\
\hline$D_{4}+A_{1}$ & $D_{4}+A_{1}$ & $\frac{31}{105}$ & \multicolumn{2}{|r|}{2.657} & \multicolumn{2}{|l|}{1.000} & \multicolumn{2}{|l|}{1.000} \\
\hline$D_{5}$ & $\left(3 A_{1}\right) "$ & $\frac{13}{42}$ & \multicolumn{2}{|r|}{2.786} & \multicolumn{2}{|l|}{1.000} & \multicolumn{2}{|l|}{2.071} \\
\hline$D_{5}$ & $\left(3 A_{1}\right)^{\prime}$ & $\frac{13}{42}$ & \multicolumn{2}{|r|}{2.786} & \multicolumn{2}{|l|}{1.000} & \multicolumn{2}{|l|}{1.839} \\
\hline$D_{5}+A_{1}$ & 0 & $\frac{13}{41}$ & \multicolumn{2}{|r|}{2.854} & \multicolumn{2}{|l|}{1.000} & \multicolumn{2}{|l|}{2.5249} \\
\hline$D_{5}+A_{1}$ & $D_{4}\left(a_{1}\right)$ & $\frac{2}{7}$ & \multicolumn{2}{|r|}{2.571} & \multicolumn{2}{|l|}{1.000} & \multicolumn{2}{|l|}{1.286} \\
\hline$D_{5}+A_{1}$ & $A_{3}+2 A_{1}$ & $\frac{2}{7}$ & \multicolumn{2}{|r|}{2.571} & \multicolumn{2}{|l|}{1.000} & \multicolumn{2}{|l|}{1.286} \\
\hline$E_{6}\left(a_{1}\right)$ & $A_{3}$ & $\frac{4}{15}$ & \multicolumn{2}{|r|}{2.400} & \multicolumn{2}{|l|}{1.000} & \multicolumn{2}{|l|}{1.400} \\
\hline$E_{6}$ & $A_{3}$ & $\frac{2}{9}$ & \multicolumn{2}{|r|}{2.000} & \multicolumn{2}{|l|}{1.000} & \multicolumn{2}{|l|}{1.667} \\
\hline$E_{7}\left(a_{1}\right)$ & $A_{2}$ & $\frac{52}{261}$ & \multicolumn{2}{|r|}{1.793} & \multicolumn{2}{|l|}{1.000} & \multicolumn{2}{|l|}{2.103} \\
\hline$E_{7}\left(a_{1}\right)$ & $4 A_{1}$ & $\frac{52}{261}$ & & 1.793 & 1.000 & & 2.253 & \\
\hline
\end{tabular}

Table 18. Plain nilpotent mass deformations of $\left(E_{7}, E_{7}\right)$ conformal matter, only rational values. 


\begin{tabular}{|c|c|c|c|c|c|c|c|c|c|}
\hline$[\mathrm{B}-\mathrm{C}]_{L}$ & {$[\mathrm{~B}-\mathrm{C}]_{R}$} & \begin{tabular}{l|l}
$r_{L}$ & $r$
\end{tabular} & $r_{R}$ & $r_{L}+r_{R}$ & \multicolumn{3}{|c|}{$a_{\mathrm{IR}}$} & $c_{\mathrm{IR}}$ & $t_{*}$ \\
\hline 0 & 0 & 0 & 0 & 0 & \multicolumn{3}{|c|}{$\frac{1745}{8}$} & $\frac{457}{2}$ & $\frac{2}{3}$ \\
\hline$A_{3}+A_{2}$ & $3 A_{1}$ & 14 & 3 & 17 & \multicolumn{3}{|c|}{$\frac{2594465245}{14362032}, \frac{325018777}{1795254}$} & $\left\{\frac{1347452419}{7181016}, \frac{676568695}{3590508}\right\}$ & $\frac{824}{1641}$ \\
\hline$D_{5}$ & 0 & 60 & 0 & 60 & \multicolumn{3}{|c|}{$\frac{88198105}{591576}, \frac{44209973}{295788}$} & $\left\{\frac{11389690}{73947}, \frac{45780601}{295788}\right\}$ & $\frac{194}{471}$ \\
\hline$E_{7}\left(a_{3}\right)$ & $2 A_{2}+A_{1}$ & 111 & 9 & 120 & \multicolumn{3}{|c|}{$\frac{12055}{96}, \frac{12091}{96}$} & $\left\{\frac{12425}{96}, \frac{12497}{96}\right\}$ & $\frac{1}{3}$ \\
\hline$E_{8}\left(b_{5}\right)$ & $D_{6}\left(a_{1}\right)$ & 1606 & 62 & 222 & \multicolumn{3}{|c|}{$\frac{823817}{8112}, \frac{103463}{1014}$} & $\left\{\frac{422939}{4056}, \frac{213413}{2028}\right\}$ & $\frac{10}{39}$ \\
\hline$D_{7}$ & $E_{6}\left(a_{1}\right)+A_{1}$ & 1828 & 85 & 267 & \multicolumn{3}{|c|}{$\frac{187823116685}{1971613488}, \frac{47191962037}{492903372}$} & $\left\{\frac{96328408265}{985806744}, \frac{12159142466}{123225843}\right\}$ & $\frac{4588}{19227}$ \\
\hline$E_{8}\left(b_{4}\right)$ & $A_{2}+A_{1}$ & 232 & 5 & 237 & \multicolumn{3}{|c|}{$\frac{1832579}{17328}, \frac{76553}{722}$} & & $\frac{16}{57}$ \\
\hline$[\mathrm{B}-\mathrm{C}]_{L}$ & {$[\mathrm{~B}-\mathrm{C}]_{R}$} & $t_{*}$ & \multicolumn{3}{|c|}{$\operatorname{Min}\left(\Delta_{\mathrm{IR}}\left(\mathrm{Z}^{\prime} \mathrm{s}\right)\right)$} & $\operatorname{Min}\left(\Delta_{\mathrm{IR}}\left(\mathcal{O}_{L}{ }^{\prime} \mathrm{s}\right)\right)$ & & & \\
\hline 0 & 0 & $\frac{2}{3}$ & \multicolumn{3}{|c|}{6.000} & 2.000 & & 2.000 & \\
\hline$A_{3}+A_{2}$ & $3 A_{1}$ & $\frac{824}{1641}$ & \multicolumn{3}{|c|}{4.519} & 1.000 & & 1.117 & \\
\hline$D_{5}$ & 0 & $\frac{194}{471}$ & \multicolumn{3}{|c|}{3.707} & 1.000 & & 2.382 & \\
\hline$E_{7}\left(a_{3}\right)$ & $2 A_{2}+A_{1}$ & $\frac{1}{3}$ & \multicolumn{3}{|c|}{3.000} & 1.000 & & 1.250 & \\
\hline$E_{8}\left(b_{5}\right)$ & $D_{6}\left(a_{1}\right)$ & $\frac{10}{39}$ & \multicolumn{3}{|c|}{2.308} & 1.000 & & 1.000 & \\
\hline$D_{7}$ & $E_{6}\left(a_{1}\right)+A_{1}$ & $\frac{4588}{19227}$ & \multicolumn{3}{|c|}{2.148} & 1.000 & & 1.000 & \\
\hline$E_{8}\left(b_{4}\right)$ & $A_{2}+A_{1}$ & $\frac{16}{57}$ & \multicolumn{3}{|c|}{2.526} & 1.000 & & 1.737 & \\
\hline
\end{tabular}

Table 19. Plain nilpotent mass deformations of $\left(E_{8}, E_{8}\right)$ conformal matter, only rational values.

\begin{tabular}{|c|c|c|c|c|c|c|c|c|c|c|}
\hline$[\mathrm{B}-\mathrm{C}]_{L}$ & {$[\mathrm{~B}-\mathrm{C}]_{R}$} & $r_{L}$ & $r_{R}$ & $r_{L}+r_{R}$ & \multicolumn{2}{|r|}{$a_{\mathrm{IR}}$} & \multicolumn{2}{|c|}{$c_{\mathrm{IR}}$} & $t_{*}$ & \\
\hline$\left[1^{8}\right]$ & {$\left[1^{8}\right]$} & 0 & 0 & 0 & & $\left\{\frac{95}{24}, \frac{41}{8}\right\}$ & \multicolumn{2}{|c|}{$\frac{31}{6}, \frac{15}{2}$} & $\frac{2}{3}$ & \\
\hline$[7,1]$ & {$\left[4^{2}\right] \mathrm{I}$} & 28 & 10 & 38 & $\left\{\frac{245}{107}\right.$ & $\left.15399, \frac{87785}{35912}\right\}$ & \multicolumn{2}{|c|}{$\frac{95905}{26934}, \frac{34961}{8978}$} & $\frac{34}{201}$ & \\
\hline$[7,1]$ & {$\left[4^{2}\right] \mathrm{II}$} & 28 & 10 & 38 & $\left\{\frac{245}{107}\right.$ & $\left.\frac{5399}{7736}, \frac{87785}{35912}\right\}$ & \multicolumn{2}{|c|}{$\frac{95905}{26934}, \frac{34961}{8978}$} & $\frac{34}{201}$ & \\
\hline$[7,1]$ & {$\left[5,1^{3}\right]$} & 28 & 10 & 38 & $\left\{\frac{245}{107}\right.$ & $\left.\frac{5399}{7736}, \frac{87785}{35912}\right\}$ & \multicolumn{2}{|c|}{$\frac{95905}{26934}, \frac{34961}{8978}$} & $\frac{34}{201}$ & \\
\hline$[\mathrm{B}-\mathrm{C}]_{L}$ & {$[\mathrm{~B}-\mathrm{C}]_{R}$} & $t_{*}$ & \multicolumn{3}{|c|}{$\operatorname{Min}\left(\Delta_{\mathrm{IR}}(\mathrm{Z}\right.$ 's $\left.)\right)$} & \multicolumn{2}{|c|}{$\operatorname{Min}\left(\Delta_{\mathrm{IR}}\left(\mathcal{O}_{L}{ }^{\prime} \mathrm{s}\right)\right)$} & \multicolumn{3}{|c|}{$\operatorname{Min}\left(\Delta_{\mathrm{IR}}\left(\mathcal{O}_{R}{ }^{\prime} \mathrm{s}\right)\right)$} \\
\hline$\left[1^{8}\right]$ & {$\left[1^{8}\right]$} & $\frac{2}{3}$ & \multicolumn{3}{|c|}{6.000} & \multicolumn{2}{|c|}{2.000} & \multicolumn{3}{|c|}{2.000} \\
\hline$[7,1]$ & {$\left[4^{2}\right] \mathrm{I}$} & $\frac{34}{201}$ & \multicolumn{3}{|c|}{1.522} & \multicolumn{2}{|c|}{1.478} & \multicolumn{3}{|c|}{1.985} \\
\hline$[7,1]$ & {$\left[4^{2}\right] \mathrm{II}$} & $\frac{34}{201}$ & \multicolumn{3}{|c|}{1.522} & \multicolumn{2}{|c|}{1.478} & \multicolumn{3}{|c|}{1.985} \\
\hline$[7,1]$ & {$\left[5,1^{3}\right]$} & $\frac{34}{201}$ & \multicolumn{3}{|c|}{1.522} & \multicolumn{2}{|c|}{1.478} & \multicolumn{3}{|c|}{1.985} \\
\hline
\end{tabular}

Table 20. Flipper field deformations of $\left(D_{4}, D_{4}\right)$ conformal matter, only rational values. 


\begin{tabular}{|c|c|c|c|c|c|c|c|c|c|}
\hline$[\mathrm{B}-\mathrm{C}]_{L}$ & {$[\mathrm{~B}-\mathrm{C}]_{R}$} & $r_{L}$ & $r_{R}$ & $r_{L}+r_{R}$ & & $a_{\mathrm{IR}}$ & & $c_{\mathrm{IR}}$ & $t_{*}$ \\
\hline 0 & 0 & 0 & 0 & 0 & & $\left\{\frac{613}{24}, \frac{691}{24}\right\}$ & & $\left.\frac{173}{6}, \frac{106}{3}\right\}$ & $\frac{2}{3}$ \\
\hline$A_{3}+A_{1}$ & $A_{1}$ & 11 & 1 & 12 & & $\left.\frac{18983}{3872}, \frac{144577}{6936}\right\}$ & $\left\{\frac{137}{69}\right.$ & $\left.\frac{7641}{936}, \frac{44453}{1734}\right\}$ & $\frac{20}{51}$ \\
\hline$D_{4}$ & $A_{3}+A_{1}$ & 28 & 11 & 39 & & $\left.\frac{5271}{400}, \frac{3223}{200}\right\}$ & & $\left.\frac{2893}{200}, \frac{1017}{50}\right\}$ & $\frac{4}{15}$ \\
\hline$D_{5}\left(a_{1}\right)$ & $A_{3}$ & 30 & 10 & 40 & & $\left.\frac{15737}{1200}, \frac{9631}{600}\right\}$ & & $\left.\frac{3651}{600}, \frac{1522}{75}\right\}$ & $\frac{4}{15}$ \\
\hline$D_{5}\left(a_{1}\right)$ & $A_{3}+A_{1}$ & 30 & 11 & 41 & & $\left.\frac{64659}{04976}, \frac{836513}{52488}\right\}$ & $\left\{\frac{749}{524}\right.$ & $\left.\frac{681}{488}, \frac{132256}{6561}\right\}$ & $\frac{64}{243}$ \\
\hline$[\mathrm{B}-\mathrm{C}]_{L}$ & {$[\mathrm{~B}-\mathrm{C}]_{R}$} & $t_{*}$ & \multicolumn{3}{|c|}{$\operatorname{Min}\left(\Delta_{\mathrm{IR}}(\mathrm{Z} ' \mathrm{~s})\right)$} & \multicolumn{2}{|c|}{$\operatorname{Min}\left(\Delta_{\mathrm{IR}}\left(\mathcal{O}_{L}{ }^{\prime} \mathrm{s}\right)\right)$} & \multicolumn{2}{|c|}{$\operatorname{Min}\left(\Delta_{\mathrm{IR}}\left(\mathcal{O}_{R}{ }^{\prime} \mathrm{s}\right)\right)$} \\
\hline 0 & 0 & $\frac{2}{3}$ & \multicolumn{3}{|c|}{6.000} & \multicolumn{2}{|l|}{2.000} & \multicolumn{2}{|c|}{2.000} \\
\hline$A_{3}+A_{1}$ & $A_{1}$ & $\frac{20}{51}$ & \multicolumn{3}{|c|}{3.529} & \multicolumn{2}{|l|}{1.000} & \multicolumn{2}{|c|}{1.824} \\
\hline$D_{4}$ & $A_{3}+A_{1}$ & $\frac{4}{15}$ & \multicolumn{3}{|c|}{2.400} & \multicolumn{2}{|l|}{1.000} & \multicolumn{2}{|c|}{1.400} \\
\hline$D_{5}\left(a_{1}\right)$ & $A_{3}$ & $\frac{4}{15}$ & \multicolumn{3}{|c|}{2.400} & \multicolumn{2}{|l|}{1.000} & \multicolumn{2}{|l|}{1.400} \\
\hline$D_{5}\left(a_{1}\right)$ & $A_{3}+A_{1}$ & $\frac{64}{243}$ & \multicolumn{3}{|c|}{2.370} & \multicolumn{2}{|l|}{1.000} & \multicolumn{2}{|l|}{1.420} \\
\hline
\end{tabular}

Table 21. Flipper field deformations of $\left(E_{6}, E_{6}\right)$ conformal matter, only rational values.

\begin{tabular}{|c|c|c|c|c|c|c|c|}
\hline$[\mathrm{B}-\mathrm{C}]_{L}$ & {$[\mathrm{~B}-\mathrm{C}]_{R}$} & $r_{L}$ & $r_{R}$ & $r_{L}+r_{R}$ & $a_{\mathrm{IR}}$ & $c_{\mathrm{IR}}$ & $t_{*}$ \\
\hline 0 & 0 & 0 & 0 & 0 & $\left\{\frac{817}{12}, \frac{589}{8}\right\}$ & $\left\{\frac{221}{3}, \frac{339}{4}\right\}$ & $\frac{2}{3}$ \\
$A_{3}+A_{2}+A_{1}$ & 0 & 15 & 0 & 15 & $\left\{\frac{1241}{24}, \frac{453}{8}\right\}$ & $\left\{\frac{661}{12}, \frac{779}{12}\right\}$ & $\frac{4}{9}$ \\
$A_{5}+A_{1}$ & $A_{2}+3 A_{1}$ & 36 & 7 & 43 & $\left\{\frac{3931}{96}, \frac{4417}{96}\right\}$ & $\left\{\frac{4163}{96}, \frac{5135}{96}\right\}$ & $\frac{1}{3}$ \\
$E_{6}\left(a_{1}\right)$ & $A_{2}+A_{1}$ & 84 & 5 & 89 & $\left\{\frac{235499}{6936}, \frac{270757}{6936}\right\}$ & $\left\{\frac{62449}{1734}, \frac{40039}{867}\right\}$ & $\frac{14}{51}$ \\
$E_{6}$ & $A_{4}+A_{1}$ & 156 & 21 & 177 & $\left\{\frac{44180297}{1642800}, \frac{1096539}{34225}\right\}$ & $\left\{\frac{23344541}{821400}, \frac{2649843}{68450}\right\}$ & $\frac{116}{555}$ \\
\hline \hline$[\mathrm{B}-\mathrm{C}]_{L}$ & {$[\mathrm{~B}-\mathrm{C}]_{R}$} & $t_{*}$ & $\operatorname{Min}\left(\Delta_{\mathrm{IR}}(\mathrm{Z}\right.$ 's $\left.)\right)$ & $\operatorname{Min}\left(\Delta_{\mathrm{IR}}\left(\mathcal{O}_{L}\right.\right.$ 's $\left.)\right)$ & $\operatorname{Min}\left(\Delta_{\mathrm{IR}}\left(\mathcal{O}_{R}\right.\right.$ 's $\left.)\right)$ \\
\hline 0 & 0 & $\frac{2}{3}$ & 6.000 & 2.000 & 2.000 \\
$A_{3}+A_{2}+A_{1}$ & 0 & $\frac{4}{9}$ & 4.000 & 1.000 & 2.333 \\
$A_{5}+A_{1}$ & $A_{2}+3 A_{1}$ & $\frac{1}{3}$ & \multicolumn{2}{|c|}{3.000} & 1.000 & 1.500 \\
$E_{6}\left(a_{1}\right)$ & $A_{2}+A_{1}$ & $\frac{14}{51}$ & \multicolumn{7}{|c|}{2.471} & 1.000 & 1.765 \\
$E_{6}$ & $A_{4}+A_{1}$ & $\frac{116}{555}$ & \multicolumn{7}{|c|}{1.881} & 1.000 & 1.432 \\
\hline
\end{tabular}

Table 22. Flipper field deformations of $\left(E_{7}, E_{7}\right)$ conformal matter, only rational values. 


\begin{tabular}{|c|c|c|c|c|c|c|c|}
\hline$[\mathrm{B}-\mathrm{C}]_{L}$ & {$[\mathrm{~B}-\mathrm{C}]_{R}$} & $r_{L}$ & $r_{R}$ & $r_{L}+r_{R}$ & $a_{\mathrm{IR}}$ & $c_{\mathrm{IR}}$ & $t_{*}$ \\
\hline 0 & 0 & 0 & 0 & 0 & $\left\{\frac{1745}{8}, \frac{5483}{24}\right\}$ & $\left\{\frac{457}{2}, \frac{1495}{6}\right\}$ & $\frac{2}{3}$ \\
\hline$A_{4}+A_{2}+A_{1}$ & $A_{4}+2 A_{1}$ & 25 & 22 & 47 & $\left\{\frac{122989}{816}, \frac{21657}{136}\right\}$ & $\left\{\frac{63463}{408}, \frac{2934}{17}\right\}$ & $\frac{20}{51}$ \\
\hline$D_{5}\left(a_{1}\right)$ & $A_{2}+3 A_{1}$ & 30 & 7 & 37 & $\left\{\frac{1200211}{7500}, \frac{632293}{3750}\right\}$ & $\left\{\frac{620893}{3750}, \frac{342634}{1875}\right\}$ & $\frac{32}{75}$ \\
\hline$D_{5}\left(a_{1}\right)+A_{2}$ & $A_{3}+A_{2}+A_{1}$ & 34 & 15 & 49 & $\left\{\frac{122683}{816}, \frac{64801}{408}\right\}$ & $\left\{\frac{63361}{408}, \frac{8785}{51}\right\}$ & $\frac{20}{51}$ \\
\hline$E_{6}\left(a_{3}\right)+A_{1}$ & $A_{2}+3 A_{1}$ & 37 & 7 & 44 & $\left\{\frac{237476949}{187894873}\right\}$ & $\{\underline{30711077}, \underline{407681569}\}$ & 180 \\
\hline & $D_{-1}^{-2}$ & 61 & 60 & 121 & 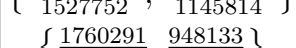 & $\begin{array}{l}190969,2291628 \\
903893 \quad 519934\end{array}$ & $\begin{array}{l}437 \\
32\end{array}$ \\
\hline$D_{5}+A_{1}$ & $D_{5}$ & 61 & 60 & 121 & $\left\{\frac{17600}{14700}, \frac{17350}{7350}\right\}$ & $\left\{\frac{x 350}{7350}, \frac{1}{3675}\right\}$ & $\frac{205}{105}$ \\
\hline$D_{6}\left(a_{1}\right)$ & $D_{5}\left(a_{1}\right)$ & 62 & 30 & 92 & $\left\{\frac{1553}{12}, \frac{6655}{48}\right\}$ & $\left\{\frac{6385}{48}, \frac{7271}{48}\right\}$ & $\frac{1}{3}$ \\
\hline$D_{6}$ & $D_{4}+A_{1}$ & 110 & 29 & 139 & $\left\{\frac{25707707}{218886}, \frac{27750643}{218886}\right\}$ & $\left\{\frac{52819073}{437772}, \frac{60990817}{437772}\right\}$ & $\frac{172}{573}$ \\
\hline$E_{8}\left(b_{5}\right)$ & $D_{4}\left(a_{1}\right)+A_{1}$ & 160 & 13 & 173 & $\left\{\frac{49357}{432}, \frac{26681}{216}\right\}$ & $\left\{\frac{25463}{216}, \frac{7367}{54}\right\}$ & $\frac{8}{27}$ \\
\hline$[\mathrm{B}-\mathrm{C}]_{L}$ & {$[\mathrm{~B}-\mathrm{C}]_{R}$} & $t_{*}$ & \multicolumn{2}{|c|}{$\operatorname{Min}\left(\Delta_{\mathrm{IR}}\left(\mathrm{Z}^{\prime} \mathrm{s}\right)\right.$} & s)) $\operatorname{Min}\left(\Delta_{\mathrm{IR}}\left(\mathcal{O}_{L}\right.\right.$ 's $\left.)\right) \mid$ & $\operatorname{Min}\left(\Delta_{\mathrm{IR}}\left(\mathcal{O}_{R}\right.\right.$ 's $\left.)\right)$ & \\
\hline 0 & 0 & $\frac{2}{3}$ & \multicolumn{2}{|r|}{6.000} & 2.000 & 2.000 & \\
\hline$A_{4}+A_{2}+A_{1}$ & $A_{4}+2 A_{1}$ & $\frac{20}{51}$ & \multicolumn{2}{|r|}{3.529} & 1.000 & 1.000 & \\
\hline$D_{5}\left(a_{1}\right)$ & $A_{2}+3 A_{1}$ & $\frac{32}{75}$ & \multicolumn{2}{|r|}{3.840} & 1.000 & 1.080 & \\
\hline$D_{5}\left(a_{1}\right)+A_{2}$ & $A_{3}+A_{2}+A_{1}$ & $\frac{20}{51}$ & \multicolumn{2}{|r|}{3.529} & 1.000 & 1.000 & \\
\hline$E_{6}\left(a_{3}\right)+A_{1}$ & $A_{2}+3 A_{1}$ & $\frac{180}{437}$ & \multicolumn{2}{|r|}{3.707} & 1.000 & 1.146 & \\
\hline$D_{5}+A_{1}$ & $D_{5}$ & $\frac{32}{105}$ & \multicolumn{2}{|r|}{2.743} & 1.000 & 1.000 & \\
\hline$D_{6}\left(a_{1}\right)$ & $D_{5}\left(a_{1}\right)$ & $\frac{1}{3}$ & \multicolumn{2}{|r|}{3.000} & 1.000 & 1.000 & \\
\hline$D_{6}$ & $D_{4}+A_{1}$ & $\frac{172}{573}$ & \multicolumn{2}{|r|}{2.702} & 1.000 & 1.000 & \\
\hline$E_{8}\left(b_{5}\right)$ & $D_{4}\left(a_{1}\right)+A_{1}$ & $\frac{8}{27}$ & \multicolumn{2}{|r|}{2.667} & 1.000 & 1.222 & \\
\hline
\end{tabular}

Table 23. Flipper field deformations of $\left(E_{8}, E_{8}\right)$ conformal matter, only rational values.

Open Access. This article is distributed under the terms of the Creative Commons Attribution License (CC-BY 4.0), which permits any use, distribution and reproduction in any medium, provided the original author(s) and source are credited.

\section{References}

[1] J.J. Heckman, D.R. Morrison, T. Rudelius and C. Vafa, Geometry of 6D RG Flows, JHEP 09 (2015) 052 [arXiv: 1505.00009] [INSPIRE].

[2] C. Cordova, T.T. Dumitrescu and K. Intriligator, Anomalies, renormalization group flows and the a-theorem in six-dimensional $(1,0)$ theories, JHEP 10 (2016) 080 [arXiv: 1506.03807] [INSPIRE].

[3] J.J. Heckman and T. Rudelius, Evidence for C-theorems in 6D SCFTs, JHEP 09 (2015) 218 [arXiv: 1506.06753] [INSPIRE].

[4] S. Cremonesi and A. Tomasiello, 6d holographic anomaly match as a continuum limit, JHEP 05 (2016) 031 [arXiv: 1512.02225] [INSPIRE].

[5] J.J. Heckman, T. Rudelius and A. Tomasiello, 6D RG Flows and Nilpotent Hierarchies, JHEP 07 (2016) 082 [arXiv: 1601.04078] [INSPIRE].

[6] N. Mekareeya, T. Rudelius and A. Tomasiello, T-branes, Anomalies and Moduli Spaces in 6D SCFTs, JHEP 10 (2017) 158 [arXiv:1612.06399] [INSPIRE]. 
[7] J.J. Heckman, T. Rudelius and A. Tomasiello, Fission, Fusion and 6D RG Flows, JHEP 02 (2019) 167 [arXiv : 1807.10274] [INSPIRE].

[8] J.J. Heckman and T. Rudelius, Top Down Approach to 6D SCFTs, J. Phys. A 52 (2019) 093001 [arXiv: 1805. 06467] [INSPIRE].

[9] J.J. Heckman, Y. Tachikawa, C. Vafa and B. Wecht, $N=1$ SCFTs from Brane Monodromy, JHEP 11 (2010) 132 [arXiv:1009.0017] [INSPIRE].

[10] J.J. Heckman and C. Vafa, An Exceptional Sector for F-theory GUTs, Phys. Rev. D 83 (2011) 026006 [arXiv: 1006.5459] [INSPIRE].

[11] S. Cecotti, C. Cordova, J.J. Heckman and C. Vafa, T-Branes and Monodromy, JHEP 07 (2011) 030 [arXiv: 1010.5780] [INSPIRE].

[12] A. Gadde, K. Maruyoshi, Y. Tachikawa and W. Yan, New N=1 Dualities, JHEP 06 (2013) 056 [arXiv: 1303.0836] [INSPIRE].

[13] P. Agarwal and J. Song, New $N=1$ Dualities from M5-branes and Outer-automorphism Twists, JHEP 03 (2014) 133 [arXiv: 1311.2945] [INSPIRE].

[14] P. Agarwal, I. Bah, K. Maruyoshi and J. Song, Quiver tails and $\mathcal{N}=1$ SCFTs from M5-branes, JHEP 03 (2015) 049 [arXiv:1409.1908] [INSPIRE].

[15] P. Agarwal, K. Intriligator and J. Song, Infinitely many $\mathcal{N}=1$ dualities from $m+1-m=1$, JHEP 10 (2015) 035 [arXiv: 1505.00255] [INSPIRE].

[16] K. Maruyoshi and J. Song, Enhancement of Supersymmetry via Renormalization Group Flow and the Superconformal Index, Phys. Rev. Lett. 118 (2017) 151602 [arXiv:1606.05632] [INSPIRE].

[17] K. Maruyoshi and J. Song, $\mathcal{N}=1$ deformations and $R G$ flows of $\mathcal{N}=2$ SCFTs, JHEP 02 (2017) 075 [arXiv: 1607.04281] [INSPIRE].

[18] P. Agarwal, K. Maruyoshi and J. Song, $\mathcal{N}=1$ Deformations and $R G$ flows of $\mathcal{N}=2$ SCFTs, part II: non-principal deformations, JHEP 12 (2016) 103 [arXiv:1610.05311] [INSPIRE].

[19] J.J. Heckman, C. Vafa and B. Wecht, The Conformal Sector of F-theory GUTs, JHEP 07 (2011) 075 [arXiv: 1103.3287] [InSPIRE].

[20] J.J. Heckman, P. Kumar, C. Vafa and B. Wecht, Electroweak Symmetry Breaking in the DSSM, JHEP 01 (2012) 156 [arXiv:1108.3849] [INSPIRE].

[21] J.J. Heckman, P. Kumar and B. Wecht, The Higgs as a Probe of Supersymmetric Extra Sectors, JHEP 07 (2012) 118 [arXiv:1204.3640] [INSPIRE].

[22] J.J. Heckman, P. Kumar and B. Wecht, Oblique electroweak parameters $S$ and $T$ for superconformal field theories, Phys. Rev. D 88 (2013) 065016 [arXiv:1212.2979] [InSPIRE].

[23] J.J. Heckman, 750 GeV Diphotons from a D3-brane, Nucl. Phys. B 906 (2016) 231 [arXiv: 1512.06773] [INSPIRE].

[24] M. Del Zotto, J.J. Heckman, P. Kumar, A. Malekian and B. Wecht, Kinetic Mixing at Strong Coupling, Phys. Rev. D 95 (2017) 016007 [arXiv: 1608.06635] [INSPIRE].

[25] K.A. Intriligator and B. Wecht, The exact superconformal R symmetry maximizes a, Nucl. Phys. B 667 (2003) 183 [hep-th/0304128] [INSPIRE].

[26] P.C. Argyres and M.R. Douglas, New phenomena in SU(3) supersymmetric gauge theory, Nucl. Phys. B 448 (1995) 93 [hep-th/9505062] [InSPIRE]. 
[27] P.C. Argyres, M.R. Plesser, N. Seiberg and E. Witten, New $N=2$ superconformal field theories in four-dimensions, Nucl. Phys. B 461 (1996) 71 [hep-th/9511154] [INSPIRE].

[28] J.A. Minahan and D. Nemeschansky, An $N=2$ superconformal fixed point with $E_{6}$ global symmetry, Nucl. Phys. B 482 (1996) 142 [hep-th/9608047] [inSPIRE].

[29] J.A. Minahan and D. Nemeschansky, Superconformal fixed points with $E_{n}$ global symmetry, Nucl. Phys. B 489 (1997) 24 [hep-th/9610076] [INSPIRE].

[30] N. Seiberg and E. Witten, Monopoles, duality and chiral symmetry breaking in $N=2$ supersymmetric QCD, Nucl. Phys. B 431 (1994) 484 [hep-th/9408099] [INSPIRE].

[31] P.S. Aspinwall and R.Y. Donagi, The heterotic string, the tangent bundle and derived categories, Adv. Theor. Math. Phys. 2 (1998) 1041 [hep-th/9806094] [INSPIRE].

[32] R. Donagi, S. Katz and E. Sharpe, Spectra of D-branes with Higgs vevs, Adv. Theor. Math. Phys. 8 (2004) 813 [hep-th/0309270] [INSPIRE].

[33] L.B. Anderson, J.J. Heckman and S. Katz, T-Branes and Geometry, JHEP 05 (2014) 080 [arXiv: 1310.1931] [INSPIRE].

[34] A. Collinucci and R. Savelli, F-theory on singular spaces, JHEP 09 (2015) 100 [arXiv: 1410.4867] [INSPIRE].

[35] A. Collinucci and R. Savelli, T-branes as branes within branes, JHEP 09 (2015) 161 [arXiv: 1410.4178] [INSPIRE].

[36] I. Bena, J. Blåbäck, R. Minasian and R. Savelli, There and back again: A T-brane's tale, JHEP 11 (2016) 179 [arXiv: 1608.01221] [INSPIRE].

[37] F. Marchesano and S. Schwieger, T-branes and $\alpha^{\prime}$-corrections, JHEP 11 (2016) 123 [arXiv: 1609.02799] [INSPIRE].

[38] L.B. Anderson, J.J. Heckman, S. Katz and L.P. Schaposnik, T-Branes at the Limits of Geometry, JHEP 10 (2017) 058 [arXiv:1702.06137] [INSPIRE].

[39] I. Bena, J. Blåbäck and R. Savelli, T-branes and Matrix Models, JHEP 06 (2017) 009 [arXiv: 1703.06106] [INSPIRE].

[40] F. Marchesano, R. Savelli and S. Schwieger, Compact T-branes, JHEP 09 (2017) 132 [arXiv: 1707.03797] [INSPIRE].

[41] M. Cvetič, J.J. Heckman and L. Lin, Towards Exotic Matter and Discrete Non-Abelian Symmetries in F-theory, JHEP 11 (2018) 001 [arXiv:1806.10594] [INSPIRE].

[42] D. Kutasov, A. Parnachev and D.A. Sahakyan, Central charges and $\mathrm{U}(1)(R)$ symmetries in $N=1$ superYang-Mills, JHEP 11 (2003) 013 [hep-th/0308071].

[43] K.A. Intriligator and B. Wecht, $R G$ fixed points and flows in $S Q C D$ with adjoints, Nucl. Phys. B 677 (2004) 223 [hep-th/0309201] [INSPIRE].

[44] M. Del Zotto, J.J. Heckman, A. Tomasiello and C. Vafa, 6d Conformal Matter, JHEP 02 (2015) 054 [arXiv: 1407.6359] [INSPIRE].

[45] J.J. Heckman, More on the Matter of 6D SCFTs, Phys. Lett. B 747 (2015) 73 [arXiv: 1408.0006] [INSPIRE].

[46] K. Ohmori, H. Shimizu, Y. Tachikawa and K. Yonekura, $6 d \mathcal{N}=(1,0)$ theories on $T^{2}$ and class S theories: Part I, JHEP 07 (2015) 014 [arXiv: 1503.06217] [INSPIRE]. 
[47] K. Ohmori, H. Shimizu, Y. Tachikawa and K. Yonekura, $6 d \mathcal{N}=(1,0)$ theories on $S^{1} / T^{2}$ and class $S$ theories: part II, JHEP 12 (2015) 131 [arXiv:1508.00915] [INSPIRE].

[48] M. Del Zotto, C. Vafa and D. Xie, Geometric engineering, mirror symmetry and $6 \mathrm{~d}_{(1,0)} \rightarrow 4 \mathrm{~d}_{(\mathcal{N}=2)}$, JHEP 11 (2015) 123 [arXiv: 1504.08348] [INSPIRE].

[49] K. Maruyoshi, E. Nardoni and J. Song, Landscape of Simple Superconformal Field Theories in 4d, Phys. Rev. Lett. 122 (2019) 121601 [arXiv:1806.08353] [INSPIRE].

[50] Y. Tachikawa and B. Wecht, Explanation of the Central Charge Ratio 27/32 in Four-Dimensional Renormalization Group Flows between Superconformal Theories, Phys. Rev. Lett. 103 (2009) 061601 [arXiv:0906.0965] [InSPIRE].

[51] N. Seiberg and E. Witten, Electric-magnetic duality, monopole condensation and confinement in $N=2$ supersymmetric Yang-Mills theory, Nucl. Phys. B 426 (1994) 19 [Erratum ibid. B 430 (1994) 485] [hep-th/9407087] [INSPIRE].

[52] D. Xie and K. Yonekura, Search for a Minimal $N=1$ Superconformal Field Theory in $4 D$, Phys. Rev. Lett. 117 (2016) 011604 [arXiv:1602.04817] [INSPIRE].

[53] S. Benvenuti and S. Giacomelli, Supersymmetric gauge theories with decoupled operators and chiral ring stability, Phys. Rev. Lett. 119 (2017) 251601 [arXiv:1706.02225] [INSPIRE].

[54] D.M. Hofman and J. Maldacena, Conformal collider physics: Energy and charge correlations, JHEP 05 (2008) 012 [arXiv: 0803.1467] [INSPIRE].

[55] D.M. Hofman, D. Li, D. Meltzer, D. Poland and F. Rejon-Barrera, A Proof of the Conformal Collider Bounds, JHEP 06 (2016) 111 [arXiv:1603.03771] [INSPIRE].

[56] S. Giacomelli, RG flows with supersymmetry enhancement and geometric engineering, JHEP 06 (2018) 156 [arXiv:1710.06469] [INSPIRE].

[57] S. Giacomelli, Infrared enhancement of supersymmetry in four dimensions, JHEP 10 (2018) 041 [arXiv: 1808.00592] [INSPIRE].

[58] M. Evtikhiev, Studying superconformal symmetry enhancement through indices, JHEP 04 (2018) 120 [arXiv: 1708.08307] [INSPIRE].

[59] C. Beasley, J.J. Heckman and C. Vafa, GUTs and Exceptional Branes in F-theory - I, JHEP 01 (2009) 058 [arXiv:0802.3391] [INSPIRE].

[60] M. Bershadsky, A. Johansen, T. Pantev and V. Sadov, On four-dimensional compactifications of F-theory, Nucl. Phys. B 505 (1997) 165 [hep-th/9701165] [INSPIRE].

[61] R. Donagi and M. Wijnholt, Model Building with F-theory, Adv. Theor. Math. Phys. 15 (2011) 1237 [arXiv:0802.2969] [INSPIRE].

[62] F. Carta, S. Giacomelli and R. Savelli, to appear.

[63] O. Aharony and Y. Tachikawa, A holographic computation of the central charges of $d=4$, $N=2$ SCFTs, JHEP 01 (2008) 037 [arXiv:0711.4532] [INSPIRE].

[64] F. Apruzzi, J.J. Heckman and T. Rudelius, Green-Schwarz Automorphisms and 6D SCFTs, JHEP 02 (2018) 157 [arXiv: 1707.06242] [INSPIRE].

[65] F. Apruzzi, J.J. Heckman, D.R. Morrison and L. Tizzano, $4 D$ Gauge Theories with Conformal Matter, JHEP 09 (2018) 088 [arXiv:1803.00582] [INSPIRE].

[66] F. Benini, Y. Tachikawa and B. Wecht, Sicilian gauge theories and $N=1$ dualities, JHEP 01 (2010) 088 [arXiv: 0909.1327] [INSPIRE]. 
[67] D. Gaiotto and S.S. Razamat, $\mathcal{N}=1$ theories of class $\mathcal{S}_{k}$, JHEP 07 (2015) 073 [arXiv: 1503.05159] [INSPIRE].

[68] S. Franco, H. Hayashi and A. Uranga, Charting Class $\mathcal{S}_{k}$ Territory, Phys. Rev. D 92 (2015) 045004 [arXiv: 1504.05988] [INSPIRE].

[69] I. Coman, E. Pomoni, M. Taki and F. Yagi, Spectral curves of $\mathcal{N}=1$ theories of class $\mathcal{S}_{k}$, JHEP 06 (2017) 136 [arXiv: 1512.06079] [INSPIRE].

[70] D.R. Morrison and C. Vafa, F-theory and $\mathcal{N}=1$ SCFTs in four dimensions, JHEP 08 (2016) 070 [arXiv : 1604.03560] [INSPIRE].

[71] J.J. Heckman, P. Jefferson, T. Rudelius and C. Vafa, Punctures for theories of class $\mathcal{S}_{\Gamma}$, JHEP 03 (2017) 171 [arXiv: 1609.01281] [INSPIRE].

[72] S.S. Razamat, C. Vafa and G. Zafrir, $4 d \mathcal{N}=1$ from 6d (1, 0), JHEP 04 (2017) 064 [arXiv: 1610.09178] [INSPIRE].

[73] I. Bah, A. Hanany, K. Maruyoshi, S.S. Razamat, Y. Tachikawa and G. Zafrir, $4 d \mathcal{N}=1$ from $6 d \mathcal{N}=(1,0)$ on a torus with fluxes, JHEP 06 (2017) 022 [arXiv:1702.04740] [INSPIRE].

[74] H.-C. Kim, S.S. Razamat, C. Vafa and G. Zafrir, E-String Theory on Riemann Surfaces, Fortsch. Phys. 66 (2018) 1700074 [arXiv:1709. 02496] [INSPIRE].

[75] F. Hassler and J.J. Heckman, Punctures and Dynamical Systems, Lett. Math. Phys. 109 (2019) 449 [arXiv:1711.03973] [InSPIRE].

[76] T. Bourton and E. Pomoni, Instanton counting in Class $\mathcal{S}_{k}$, arXiv:1712.01288 [INSPIRE].

[77] H.-C. Kim, S.S. Razamat, C. Vafa and G. Zafrir, D-type Conformal Matter and SU/USp Quivers, JHEP 06 (2018) 058 [arXiv: 1802.00620] [INSPIRE].

[78] S.S. Razamat and G. Zafrir, Compactification of 6d minimal SCFTs on Riemann surfaces, Phys. Rev. D 98 (2018) 066006 [arXiv:1806.09196] [INSPIRE].

[79] O. Chacaltana, J. Distler and Y. Tachikawa, Nilpotent orbits and codimension-two defects of 6d $N=(2,0)$ theories, Int. J. Mod. Phys. A 28 (2013) 1340006 [arXiv:1203.2930] [InSPIRE].

[80] A.T. Rakotoarisoa, The Bala-Carter Classification of Nilpotent Orbits of Semisimple Lie Algebras, Ph.D. Thesis, University of Ottawa, Canada, (2017).

[81] N. Haouzi and C. Schmid, Little String Defects and Bala-Carter Theory, arXiv:1612.02008 [INSPIRE].

[82] R. Feger and T.W. Kephart, LieART - A Mathematica application for Lie algebras and representation theory, Comput. Phys. Commun. 192 (2015) 166 [arXiv:1206.6379] [INSPIRE].

[83] O. Chacaltana, J. Distler and A. Trimm, Tinkertoys for the $E_{6}$ theory, JHEP 09 (2015) 007 [arXiv: 1403.4604] [INSPIRE].

[84] O. Chacaltana, J. Distler, A. Trimm and Y. Zhu, Tinkertoys for the E $E_{7}$ theory, JHEP 05 (2018) 031 [arXiv: 1704.07890] [INSPIRE].

[85] O. Chacaltana, J. Distler, A. Trimm and Y. Zhu, Tinkertoys for the E $E_{8}$ Theory, arXiv:1802.09626 [INSPIRE].

[86] K. Ohmori, H. Shimizu, Y. Tachikawa and K. Yonekura, Anomaly polynomial of general $6 d$ SCFTs, PTEP 2014 (2014) 103B07 [arXiv: 1408.5572] [InSPIRE].

[87] P. Agarwal, K. Maruyoshi and J. Song, unpublished. 